Portland State University

PDXScholar

Summer 7-26-2017

\title{
Properties of Carbon Nanotubes: Defects, Adsorbates, and Gas Sensing
}

Micah C. Eastman

Portland State University

Follow this and additional works at: https://pdxscholar.library.pdx.edu/open_access_etds

Part of the Physics Commons

Let us know how access to this document benefits you.

\section{Recommended Citation}

Eastman, Micah C., "Properties of Carbon Nanotubes: Defects, Adsorbates, and Gas Sensing" (2017). Dissertations and Theses. Paper 3775.

https://doi.org/10.15760/etd.5659

This Dissertation is brought to you for free and open access. It has been accepted for inclusion in Dissertations and Theses by an authorized administrator of PDXScholar. Please contact us if we can make this document more accessible: pdxscholar@pdx.edu. 
Properties of Carbon Nanotubes: Defects, Adsorbates, and Gas Sensing

by

Micah C. Eastman

A dissertation sumbitted in partial fullfillment of the requirements for the degree of

Doctor of Philosophy

in

Applied Physics

Dissertation Committee:

Jun Jiao, Chair

Bin Jiang

Erik Sanchez

Raj Solanki

Tami Clare

Portland State University

2017 


\begin{abstract}
Carbon naotubes and graphene have been a trending research topic in the past decade. These graphitic compounds exhibit numerous advantageous properties (electronic, mechanical, thermal, optical, etc) which industry and researchers alike are excited to take advantage of. Beyond the challenges of yield and controlled growth, there are a number of standing questions which govern some of the more fundamental characteristics of these materials: What role do lattice defects play in the adsorption of gas molecules on the surface of carbon nanotubes? How are the electronic states of the carbon nanotubes influenced by these adsorbed molecules? And how can we develop models to predict useful applications of this knowledge?

In order to address these questions, this study combines Raman spectroscopy and electronic measurements carried out in highly controlled environments of carbon nanotube transistors. Assessing these data in conjunction shows that the defect density of a carbon nanotube channel has no correlation with observed threshold voltage shifts, or change in Schottky barrier, due to the presence of ambient oxygen. With these insights in mind, a dynamic adsorption-desorption model is proposed which addresses the oxygen sensitivity of carbon nanotube transistors. Instrumentation and computational developments which facilitated these measurements are also disclosed.
\end{abstract}




\section{Acknowledgements}

I would like to thank my research advisor, Prof. Jun Jiao, for her continued support and guidance over the course of my graduate studies and my thesis committee for their time, interest, and input on my project. I am also thankful of the entire Department of Physics faculty, support staff, and graduate student body for their friendship and kind assistance which always seemed to be in ample supply.

I will always be grateful for the seeminly inexhaustible patience of my wife, Meghann Eastman, who is the greatest companion I could hope to have in life; and for the rest of my family, which has ever been a source of love and levity.

Portions of this work have been supported by Intel Corporation and the National Science Foundation (NSF) under award no. 1057565 and REU site no. 1004737. Financial support for acquisition of instrumentation was also provided in part by the Oregon Nanoscience and Microtechnologies Institute (ONAMI). 


\section{Table of Contents}

Abstract $\quad$ i

Acknowledgements $\quad$ ii

List of Tables $\quad$ V

List of Figures $\quad$ vi

List of Abbreviations/Symbols $\quad$ ix

1 Introduction $\mathbf{1}$

1.1 Applications of carbon nanotubes . . . . . . . . . . . . . 1

1.2 Current literature: Status and limitations . . . . . . . . . . . . . 3

1.3 Approach \& organization . . . . . . . . . . . . . . . 4

2 Experimental Concepts $\quad 8$

2.1 Physical and electronic structure of carbon nanotubes . . . . . . . . 8

2.2 Raman scattering in graphitic compounds . . . . . . . . . . . . . . 14

2.3 Carbon nanotube field-effect transistors . . . . . . . . . . . . . . . . . 17

2.4 Charge carrier transport . . . . . . . . . . . . . . . . 20

3 Experimental Methods 23

3.1 Quantitative Raman spectroscopy . . . . . . . . . . . . . . . . 23

3.2 Polarization-dependent Raman spectroscopy . . . . . . . . . . . . . 25

3.3 Electron transport measurements . . . . . . . . . . . . . . . . 29

3.4 Electrical impact of adsorbates . . . . . . . . . . . . . . . 30

4 Instrumentation Developments $\quad 34$

4.1 Raman spectrometer . . . . . . . . . . . . . . . . 34

4.1.1 Motorized half-wave plate . . . . . . . . . . . . . . 35

4.1.2 Quarter-wave plate optics . . . . . . . . . . . . 36

4.2 Micro-environmental probe station . . . . . . . . . . . . . . . 38

4.2.1 Gas injection and sensing .............. 39

5 Computational Methods $\quad 42$

5.1 Raman region identification . . . . . . . . . . . . . . . 43

5.2 Raman map stitching . . . . . . . . . . . . . . . . . . . . . . . . . . . . . . . . . 45

5.3 Effective Schottky barrier . . . . . . . . . . . . . 49 
6 Results $\quad 53$

6.1 Device fabrication . . . . . . . . . . . . . . . . . 53

6.2 Raman spectroscopy . . . . . . . . . . . . . . . . . . . . . 54

6.2 .1 Defect mapping . . . . . . . . . . . . . . . . . 54

6.2 .2 Defect quantification . . . . . . . . . . . . . . . 55

6.3 Electron transport . . . . . . . . . . . . . . . . . . 57

6.4 Correlations . . . . . . . . . . . . . . . . . . . . . . 59

7 Discussion $\quad 62$

7.1 Defect density estimation . . . . . . . . . . . . . . . . . 62

7.2 Gas-surface collisions . . . . . . . . . . . . . . . . 65

7.3 Chemisorption and physisorption processes . . . . . . . . 66

7.4 Dynamic nanotube-adsorbate model . . . . . . . . . . . . . . . 68

7.5 Charge traps and transport . . . . . . . . . . . . . 72

8 Conclusion $\quad 74$

8.1 Broader impact . . . . . . . . . . . . . . . . 76

8.2 Further studies . . . . . . . . . . . . . . . . . . . . 77

8.2.1 Differentiation: metallic vs. semiconducting . . . . . . . 77

8.2 .2 Photostimulated transport . . . . . . . . . . . . . . . 78

8.2 .3 Kelvin probe measurements . . . . . . . . . . . . . . . 79

$\begin{array}{ll}\text { References } & 80\end{array}$

Appendix A Python code $\quad 85$

A.1 Classes . . . . . . . . . . . . . . . . . . . . . . 85

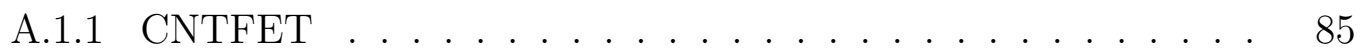

A.1.2 MEPS . . . . . . . . . . . . . . . . . . 86

A.1.3 GateSweep . . . . . . . . . . . . . . . . . . . . . . . . . . . . 89

A.1.4 Raman . . . . . . . . . . . . . . . . . . . . . . . . 93

A.2 Functions . . . . . . . . . . . . . . . . . . . . 102

A.2.1 Curve fitting . . . . . . . . . . . . . . . . . . . . 102

A.2.2 Raman map stitching . . . . . . . . . . . . . . . . . . . 102

A.3 Code blocks . . . . . . . . . . . . . . . . . . . . . 103

A.3.1 Raman map stitching . . . . . . . . . . . . . . . . . . . . 104

$\begin{array}{ll}\text { Appendix B Publications } & 108\end{array}$

Journal Publications . . . . . . . . . . . . . . . . . . . . . . . 108

Conference Proceedings . . . . . . . . . . . . . . . . . . 108

Co-authored Publications . . . . . . . . . . . . . . . 108 


\section{List of Tables}

Table 1: Collision rates of $\mathrm{O}_{2}$ molecules at various pressures with entire CNT channel and with defect sites. . . . . . . . . . . . . 66 


\section{List of Figures}

Figure 1: Flow diagram of experimental approach and techniques applied. 5

Figure 2: (a) Unit cell and (b) first Brillouin zone of monolayer graphene 8

Figure 3: (a) Pristine graphitic carbon and (b) monovacancy, (c) divacancy, and (d) Stone-Wales defects . . . . . . . . . . . . . . . . . 9

Figure 4: Schematic of 2D basis for CNTs with chirality boundary points labeled. Red indicates semiconducting chiralities, and blue metallic. . 10

Figure 5: Example unit cell of a $(4,2) \mathrm{CNT}$ with chiral and translational vectors labeled. . . . . . . . . . . . . . . . . . . .

Figure 6: (a) Progression of DoS for $(2,0)$, (4,0), and $(8,0)$ CNTs showing progression of vHSs. (b) Katura plot showing vHS positions as a function of CNT diameter (red: semiconducting, blue: metallic) . . . 12

Figure 7: Phonon dispersion relation of monolayer graphene. . . . . . . . 15

Figure 8: Raman spectrum of a single CNT with D, G, and 2D peaks labeled. 16

Figure 9: (a) Band diagrams of metals and semiconductors and their junctions (b) according to the Schottky-Mott model and (c) with potential Fermi level pinning effects. . . . . . . . . . . . . . . .

Figure 10: Basic (a) physical architecture and (b) band structure schemes of a CNT-FET device. . . . . . . . . . . . . . . . . .

Figure 11: Example schematic of hole injection in a Schottky barrier FET under various operating conditions (a) unbiased, off state, (b) biased, off state, (c) biased, threshold state with thermionic emission, (d) biased, post-threshold/on state with thermionic and field emission. Source and drain electrodes are designated by "S" and "D," respectively. 21

Figure 12: Gate-source characteristics of a single CNT showing majority electron charge transport regimes. . . . . . . . . . . . . .

Figure 13: Raman G-band spectrum of a single CNT (a) with polarization vector varied from $0-90^{\circ}$ relative to the $\mathrm{CNT}$ axis and (b) relative intensity when collected with aligned polarization and CRP.

Figure 14: Raman D and G peak intensities of a single CNT plotted with respect to (a) polarization angle, compared against a $\cos ^{2} \theta$ trendline, and (b) to each other showing that the D/G ratio is linear and independent of polarization. . . . . . . . . . . . . . .

Figure 15: (a) SEM image of a CNT-FET with region of interest indicated. Corresponding Raman spectrum maps of the RBM peak intensity using (b) a horizontally and (c) vertically polarized source . . . . . . . . . .

Figure 16: Gate-source characteristics of (a) semiconducting and (b) metallic CNTs with $V_{D S}$ varied from $-1 \mathrm{~V}$ to $1 \mathrm{~V}$ in $250 \mathrm{mV}$ increments. . . 
Figure 17: Gate sweeps of a single CNT with $V_{D S}$ varied from $-1 \mathrm{~V}$ to $1 \mathrm{~V}$ in $250 \mathrm{mV}$ increments at approximately (a) $10^{-9}$, (b) $10^{-4}$, and (c) $10^{0}$ Torr chamber pressure. (d) Single gate sweeps at each pressure setting shown for comparison $\left(V_{D S}=1 \mathrm{~V}\right)$. Dashed lines indicate locations of $I_{D S}$ minima. . . . . . . . . . . . . . . . . .

Figure 18: (a) Intrinsic CNT-electrode junction with relevant quantities labeled. Increases in Schottky barrier height caused by (b) a change in the electrode work function and (c) a change in CNT electron affinity.

Figure 19: Optical pathway of the HR800 system with various optical components labeled: filters (F1, F2), mirrors (M1-M5), neutral-density filter wheel (NDF), half-wave plate mount (HWP), and motor control unit $(\mathrm{MCU}) \ldots \ldots \ldots \ldots$

Figure 20: (a) Exploded diagram of motorized HWP system (photograph visible in Figure 19). (b) Software control interface (live CCD imaging window not shown). . . . . . . . . . . . . . . . . .

Figure 21: Optical pathway of the HR800 system with QWP mount inserted showing the cleanup polarizer, P1, and the calibration polarizer, P2, which is not utilized during measurements. . . . . . . . . . . . .

Figure 22: MEPS chamber overview with accessories labeled. . . . . . . .

Figure 23: Analog partial pressure histogram scan before and immediately after bake-out and photodesorption process with well known gas species labeled.

Figure 24: Gas concentration curves as measured by the RGA as $\mathrm{O}_{2}$ is injected into the system at approximately $t=0.5 \mathrm{hrs}$. Mass-charge ratios are indicated in the legend. . . . . . . . . . . . . . . . .

Figure 25: Averaged reference spectrum of a CNT used for PCA of all Raman maps . . . . . . . . . . . . . . . . . . . .

Figure 26: Overlapping Raman D-band intensity maps, (a) $M_{1}$ and (b) $M_{2}$ for a single device before any compositing function is applied.

Figure 27: Composite Raman maps of D-band intensity merged by (a) direct overlay and (b) by the described map stitching algorithm. . . .

Figure 28: Location of thermionic emission regime in a single gate-source curve. (a) Transconductance, as determined by and iterated 20-channel linear fit with the thermioninc cut-off indicated and (b) linear fit of the defined regime in the full gate-source curve. . . . . . . . . . . . .

Figure 29: Logarithmic plot of drain-source current characteristics at a fixed gate voltage in the thermionic emission regime. Extrapolation of saturation current from the linear regime (on logarithmic scale) is illustrated. . . . . . . . . . . . . . . . . . 
Figure 30: (a) Grid of sixteen CNT-FETs and accompanying test/alignment pads. (b) A single CNT-FET channel with contrast-enhanced channel showing bridging CNT (scale bar corresponds with the host image). . . . . . . . . . . . . . . . . . . . .

Figure 31: Raman spectrum maps of a CNT-FET collected with a circularly polarized source showing (a) D and (b) G peak integrated intensities. (c) Corresponding D/G ratio map. . . . . . . . . . . . . . . . .

Figure 32: Histogram of $\mathrm{D} / \mathrm{G}$ ratio of an individual $\mathrm{CNT}$ channel with (a) broad distribution and (b) narrow distribution of defects. . . . . . . .

Figure 33: Histogram of (a) average D/G ratio and (b) number of Raman map points for all CNT-FETs included in this study. . . . . . . . . .

Figure 34: Comparison of threshold and off-state gate voltages for each CNT-FET at (a) $3.5 \times 10^{-9}$, (b) $3.0 \times 10^{-4}$, and (c) $1.0 \times 10^{0}$ Torr chamber pressure $\left(V_{D S}=250 \mathrm{mV}\right)$. (d) Centroid of $(\mathrm{a}-\mathrm{c})$. Error bars indicate one standard deviation centered about the average $V_{G o}$ and

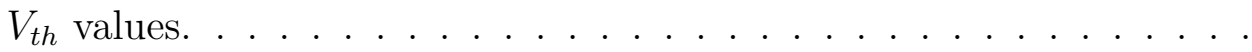

Figure 35: (a) Average change in $V_{G o}$ and $V_{G k}$ with respect to chamber pressure and (b) corresponding changes for the two chamber pressure transitions $\left(3.5 \times 10^{-9}\right.$ to $3.0 \times 10^{-4}$ Torr and $3.0 \times 10^{-4}$ to $1.0 \times 10^{0}$ Torr). . . . . . . . . . . . . . . . .

Figure 36: Correlative data showing relationship between (a) gate voltage off-state shift and (b) threshold voltage shift as a function of Raman map points for both chamber pressure transitions $\left(3.5 \times 10^{-9}\right.$ to $3.0 \times$ $10^{-4}$ Torr and $3.0 \times 10^{-4}$ to $1.0 \times 10^{0}$ Torr). (c) Similar data showing the relationship between gate voltage off-state shift and (d) threshold voltage shift as a function of Raman $\mathrm{D} / \mathrm{G}$ ratio. . . . . . . . . . . .

Figure 37: Correlative data showing relationship between change in effective Schottky barrier and (a) number of Raman map points and (b) Raman D/G ratio for both chamber pressure transitions $\left(3.5 \times 10^{-9}\right.$ to $3.0 \times 10^{-4}$ Torr and $3.0 \times 10^{-4}$ to $1.0 \times 10^{0}$ Torr). Error bars represent one standard deviation. . . . . . . . . . . . . . . .

Figure 38: (a) Cross-section of custom feed-through with Teflon ferrule. Photographs of (a) optical fiber feed-through and (b) clamping fiber armature above a specimen. . . . . . . . . . . . . . .

Figure 39: Schematic of (a) Kelvin probe feed-through and (b) control and measurement electronics. . . . . . . . . . . . . . . . 


\section{List of Abbreviations/Symbols}

\section{Abbreviations}

$\begin{array}{lr}\text { A/O } & \text { acoustic/optic (phonon mode) } \\ \text { CCD } & \text { charge-coupled device } \\ \text { CNT } & \text { carbon nanotube } \\ \text { CRP } & \text { continuously rotating polarization } \\ \text { CVD } & \text { chemical vapor deposition } \\ \text { DoS } & \text { density of states } \\ \text { FET } & \text { field-effect transistor } \\ \text { FTIR } & \text { Fourier-transform infrared } \\ \text { HWP } & \text { half-wave plate } \\ \text { i/o } & \text { infrared } \\ \text { IR } & \text { red } \\ \text { L/T } & \text { motor control unit } \\ \text { MCU } & \text { multi-walled } \\ \text { MEPS } & \text { numerical aperture } \\ \text { MW } & \text { in-plane/out-of-plane (phonon mode) } \\ \text { NA } & \text { neutral density filter } \\ \text { NDF } & \text { longitudinal/transverse (phonon mode) } \\ \text { PCA } & \text { micro-environmental probe station } \\ \text { PECVD } & \text { plasma-enhanced chemical vapor deposition } \\ \text { QWP } & \text { quarter-wave plate } \\ \text { RBM } & \text { radial breathing mode } \\ \text { RGA } & \text { residual gas analyzer } \\ \text { SEM } & \text { shincipal component analysis } \\ \text { SW } & \text { ultraviolet } \\ \text { UV } & \text { ultra-high vacuum } \\ \text { UHV } & \text { van Hove singularity } \\ \text { vHS } & \text { scanning electron microscopy }\end{array}$




\section{Symbols}

\begin{tabular}{|c|c|}
\hline $\overrightarrow{a_{1}}, \overrightarrow{a_{2}}$ & unit cell basis vectors (p. 10) \\
\hline$A_{G}$ & Richardson constant (p. 21) \\
\hline $\overrightarrow{c_{h}}$ & carbon nanotube chiral vector (p. 10) \\
\hline $\mathrm{D}$ & Raman D peak (p. 15) \\
\hline$d_{t}$ & carbon nanotube diameter (p. 10) \\
\hline$E_{d}$ & desorption activation energy (p. 69) \\
\hline$E_{\text {laser }}$ & Raman laser excitation energy (p. 24) \\
\hline$E_{n n}$ & carbon nanotube electronic energy transition (p. 12) \\
\hline$E_{q}$ & charge carrier energy (p. 69) \\
\hline G & Raman G peak (p. 15) \\
\hline$g$ & transconductance (p. 49) \\
\hline$h$ & Planck constant (p. 70) \\
\hline$I$ & intensity (p. 26) \\
\hline$I_{D S}$ & drain current (p. 18) \\
\hline$I_{S}$ & saturation current (p. 51) \\
\hline$J$ & current density (p. 21) \\
\hline$J_{S}$ & saturation current density (p. 21) \\
\hline$k_{B}$ & Boltzmann constant (p. 21) \\
\hline$k_{n m}$ & cross-correlation coefficient (p. 43) \\
\hline$L_{D}$ & mean distance between lattice defects (p. 63) \\
\hline$\left(n_{1}, n_{2}\right)$ & carbon nanotube chiral indices (p. 10) \\
\hline$n_{d}$ & dopant concentration per unit area (p. 69) \\
\hline$n_{D}$ & defect density per unit area (p. 63) \\
\hline$N_{C}$ & number of pristine carbon sites (p. 64) \\
\hline$N_{D}$ & number of atomic defect sites (p. 64) \\
\hline$N_{L}$ & number of available adsorption sites (p. 69) \\
\hline$N_{\mathrm{O}_{2}}$ & number of occupied adsorption sites (p. 69) \\
\hline$N_{T}$ & number of potential adsorption sites (p. 69) \\
\hline$N_{v}$ & gas molecules per unit volume (p. 65) \\
\hline$P$ & chamber pressure (p. 51) \\
\hline$q$ & charge (p. 21) \\
\hline$s$ & sticking coefficient (p. 69) \\
\hline$\vec{T}$ & carbon nanotube unit cell translation vector (p. 11) \\
\hline$t_{a c q}$ & Raman acquisition time (p. 26) \\
\hline $\bar{v}$ & average gas molecule velocity (p. 65) \\
\hline$V_{D S}$ & drain source voltage (p. 17) \\
\hline$V_{G}$ & gate voltage (p. 17) \\
\hline$V_{t h}$ & threshold voltage (p. 49) \\
\hline$V_{G o}$ & off-state gate voltage (p. 58) \\
\hline$z$ & gas molecule collusion rate (p. 66) \\
\hline
\end{tabular}


$\Delta \Phi_{S B} \quad$ change in effective Schottky barrier (p. 51)

$\theta \quad$ polarization angle (p. 26)

$\pi, \pi^{*} \quad$ bonding and anti-bonding pi-orbitals (p. 15)

$\sigma \quad$ standard deviation (p. 44)

$\nu \quad$ pre-exponential frequency factor (p. 69)

$\phi \quad$ gas molecule flux (p. 65)

$\phi_{m} \quad$ metal work function (p. 18)

$\Phi_{S B} \quad$ effective Schottky barrier (p. 18)

$\chi \quad$ semiconductor electron affinity (p. 18)

$\omega_{R B M} \quad$ radial breathing mode frequency (p. 24) 


\section{Introduction}

Since the discovery of the carbon nanotube (CNT) [1], it has been a topic of great interest for material scientists and nanodevice engineers alike. Its unique quasi-1D structure and $s p^{2}$ bonding results in a number of interesting optoelectronic and mechanical properties. This has has led to investigation of their capabilities as transistors, field-emission electron sources, interconnects, manipulators, heatsinks, and high-tensile fibers, as well as in a variety of composite material and sensing platforms $[2-8]$. To address the broader impact of this study, the subsequent section discusses some of these innovations in electronic CNT-based devices.

\section{Applications of carbon nanotubes}

One of the more promising and advanced applications for CNTs is in field-emission electron sources $[9,10]$. Due to the reliability and ease of fabrication of highly-aligned multi-walled CNTs by plasma-enhanced chemical vapor deposition (PECVD), fieldemitters are the most mature CNT-based technology. They have been fabricated by a wide-variety of methods and in range of architectures [11-13]. Due to the high aspect-ratios of CNTs, their promise in this application was immediately foreseen. Their robustness, both mechanical and thermal, has been a further benefit to their promise in this field.

The properties which make CNTs advantageous as electron sources also favorable for vertical interconnects in integrated circuits. Several studies have demon- 
strated the use of individual and bundled vertically-aligned CNTs as interconnecting circuits [14]. Li et al have also demonstrated the practicality of traditional chemical mechanical planarization, an obligatory polishing method used in multi-layer device fabrication, to produce CNT interconnect layers [15]. However, a standing difficulty with their application in this field lies in the reliable production of low-resistance ohmic contacts. Advancement of our understanding concerning CNT surface interactions, especially with respect to defects, is paramount towards addressing the problems with CNT contacts.

CNTs have also been heavily studied as candidates for next-generation fieldeffect transistors (FETs) [16]. Due to the $s p^{2}$ bonding of the carbon atoms, conduction band electrons have drastically reduced scattering as they traverse a CNT channel. Indeed, the ballistic transport limit of CNTs have been realized and incredibly promising prototype devices have been evaluated [17]. Two principle challenges exist with the CNT-FET commercialization transition: the previously mentioned difficulty in fabricating reliable contacts, and growth of CNTs with controlled chirality (and thereby electrical performance). While this study does not build upon our understanding of the CNT growth process, it does provide a variety of new measurement techniques which can be invaluable for large-scale testing and evaluation of CNT-FETs.

Building upon the FET platform, CNTs have also been studied as sensors. As this study focuses on the electrical impact of ambient gas environments, it is essential to acknowledge their role as a sensor platform (specifically gas sensors). 
There have been numerous studies describing the effectiveness of CNTs as gas sensors

[18-20]. A notion cited almost universally in this literature is that surface defects play a substantial role in the chemisorption of gas molecules, and thereby electrical sensitivity. While this hypothesis is sensible, it has not undergone sufficient scrutiny. This aspect of CNT surface-science is foundational to their development as a sensor platform and is of primary interest in this study. While this is presented in context of CNT-based gas sensors, these issues carry over towards the emerging and compelling field of CNT-based biosensors, bioprobes, and other composite-based sensors.

\section{Current literature: Status and limitations}

While there have been substantial developments in CNT-based devices, as discussed, there are numerous fundamental challenges which must be addressed in order to bridge the gap to CNT commercialization. As mentioned in the abstract, we seek to specifically address the impact of lattice defects on adsorption and the effects of these adsorbates upon the electronic states of the CNT.

Concerning the nature of defect quantification in CNTs by Raman spectroscopy, a substantial body of work has been established [21-23]. These studies systematically address a variety of defect-centric questions about CNTs (some of which are discussed in further detail in section Section 3.1). However, very few studies address the need for Raman mapping techniques [24-26]. Comprehensive methods for defect mapping, which have direct and profound implications on CNT-based electronics, are absent 
from this field of study. From this applications standpoint, equal emphasis, if not greater, should be placed upon defect mapping. In this study, we not only develop mapping and quantification techniques, but also apply these techniques to assess the functional role of lattice defects in CNTs in the adsorption process.

Similarly, the electronic properties of CNTs have been well explored, and their response to ambient gases documented. What is lacking, however, is an in-depth analysis and correlative study which can affirm or disprove the mechanisms proposed in the literature which give rise to these electronic phenomena. Indeed, much of the CNT gas sensing studies focus solely on efficacy and device performance, rather than assessing root causes for transport characteristics caused by ambient gases.

\section{Approach \& organization}

This study employs a two-pronged approach to characterize the defect-dependent adsorption and gas sensing of CNT-FETs. The two main experimental thrusts are Raman spectroscopy (an intrinsic material evaluation) and electronic characterizations (which assess device performance) as shown in Figure 1. Using Raman spectroscopy, the polarization-dependent nature of CNTs will be explored and CNT channels will be mapped and analyzed to quantify the number of lattice defects. The electronic characterizations will include gate-source device characteristics with varied ambient pressures. This will allow extraction of critical device parameters which can be correlated against the defect quantification data. In combining these two data, it will be 
possible to determine the role of defects in adsorption and to develop models for the CNT-adsorbate interactions.

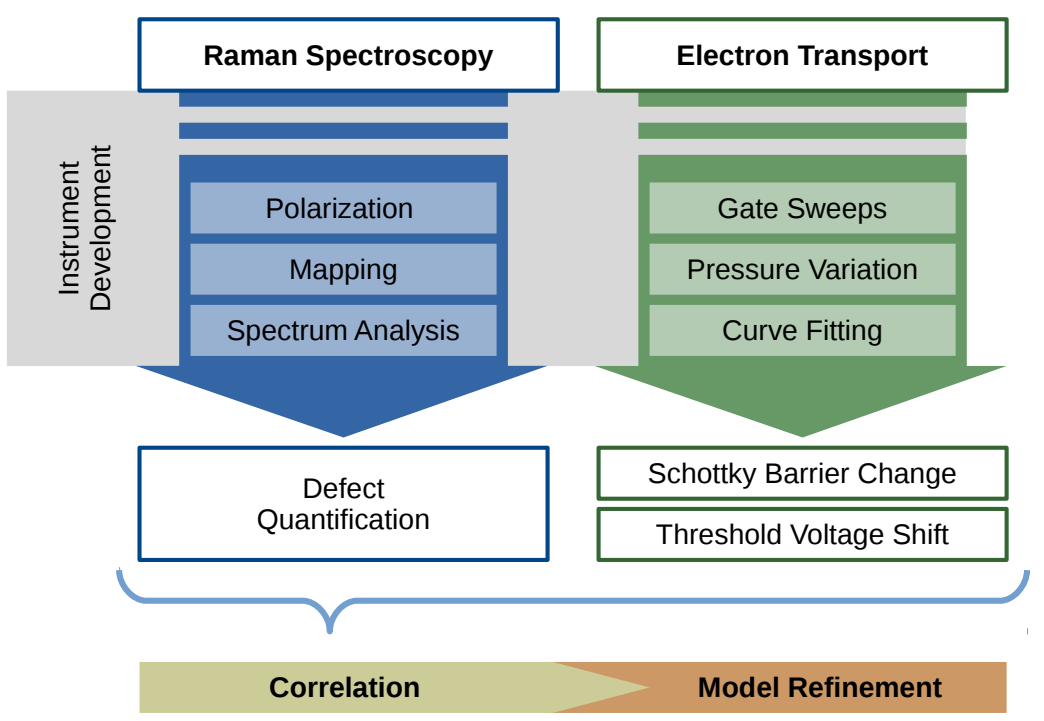

Figure 1: Flow diagram of experimental approach and techniques applied.

To develop a foundational understanding of the materials presented herein, it is essential to first consider the physical and electronic structure of CNTs. These concepts provide the basis for all observations and must be carefully considered in order to interpret results. While the conceptual links between physical structure and lattice defects (as investigated by Raman spectroscopy) or electronic structure and electronic characteristics may be most evident, there are other important phenomena to consider. Raman scattering events, for instance, also depend upon the electronic structure of the CNT and polarization-dependent effects can not be explained by the physical structure alone. 
Following this discussion of fundamental material properties, concepts relevant to the experimentally observed phenomena are introduced. The Raman peaks of interest and corresponding mechanisms are enumerated and basic quantification methodologies are covered. In addition, CNT-FET device architecture is conceptually introduced in terms of the electronic band structure and metal-semiconductor junctions and the corresponding implications for charge transport mechanisms. As this device will be the platform for studying CNTs, these points will be critical in interpreting data and forming meaningful correlations.

In the following sections, these basic physical principals will be cultivated into experimental methods which will guide the study as a whole. Requirements and challenges of Raman spectroscopy of CNTs and a basis for defect quantification and mapping will be covered. Similarly, the general gate-source trends in response to adsorbates will be illustrated and the hypothesized mechanisms for this response will be outlined. As the objectives of this study mandate new measurement techniques, the instrumentation developments carried out over the course of these experiments are also discussed.

Once the nature and mechanism of the measurements are discussed, it is possible to outline the computational methods employed which will translate these measurements into quantities of interest. Since this study focuses on forming larger scale correlations between numerous measurements, the computational works are relatively extensive. In the Results and Discussion sections, a summary of the statistics and 
correlations will be presented.

In the concluding remarks of this work, the important results will be discussed and summarized in relation to the hypothesized mechanisms for the adsorbate-CNT interactions. In addition, limitations, challenges, and opportunities for expanding these works will be explored. 


\section{Experimental Concepts}

\section{Physical and electronic structure of carbon nanotubes}

Bearing the previously presented dilemmas of CNT-FET operation in mind, we must have a complete understanding of CNT structure (physical and electronic). Only then is it possible to design an experiment to test these electronic phenomena and provide meaningful interpretations. Since CNTs are readily visualized as a rolled graphene sheet, graphene is the natural starting point for a discussion of structure. Indeed, throughout this study, graphene will continue to be the basis for the most foundational knowledge of CNTs. Graphene is a 2D sheet consisting of a hexagonallyarranged sheet of $s p^{2}$ bonded carbon atoms [27-29]. The corresponding unit cell and first Brillouin zone is depicted in Figure 2.

(a)

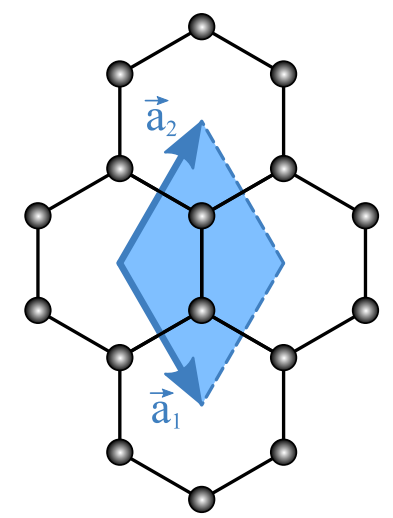

(b)

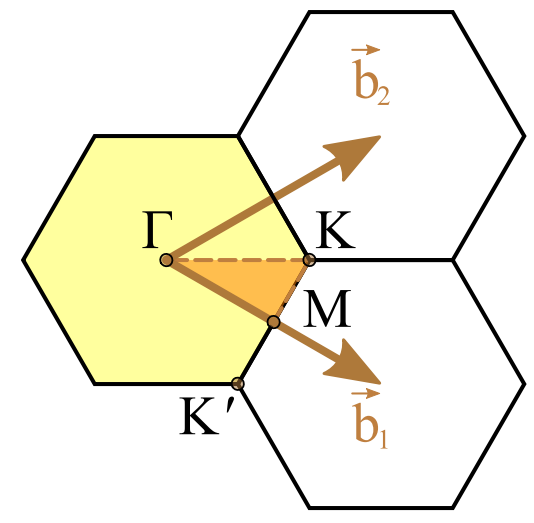

Figure 2: (a) Unit cell and (b) first Brillouin zone of monolayer graphene

When discussing structure, it is also important to develop an understanding of crystalline defects (as previously mentioned, this study will focus on the nature of these defects). There are several principal lattice formations which will contribute 
towards future discussions of defects. These include: (1) single-atom vacancies (monovacancies), (2) two-atom vacancies (divacancies), (3) Stone-Wales defects (in which a single bonded pair of carbon atoms is rotated $90^{\circ}$ ), and (4) $s p^{3}$ coordinated carbon atoms (out-of-plane amorphous clusters) [30-32]. The first three lattice defects are represented in Figure 3. The principle impact of these defects is in Raman spectroscopy and will therefore be discussed in greater detail in later sections.

(a)

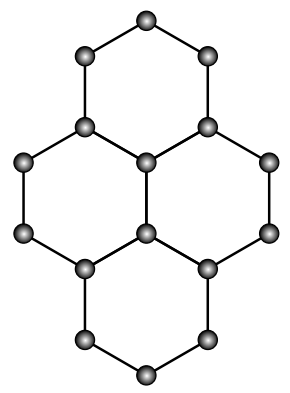

(b)

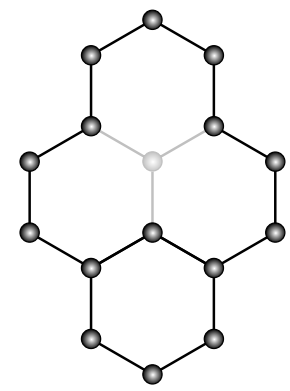

(c)

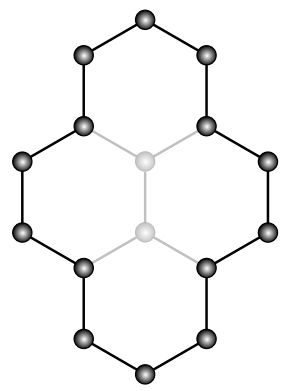

(d)

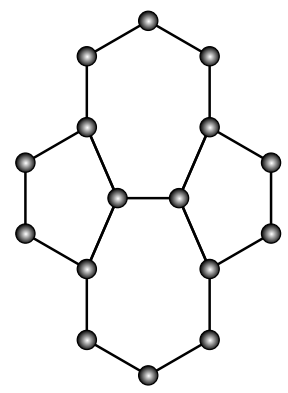

Figure 3: (a) Pristine graphitic carbon and (b) monovacancy, (c) divacancy, and (d) Stone-Wales defects

As previously stated, the structure of CNTs can be readily visualized as a rolled graphene sheet. The complete description of the CNT thereby requires specification of the structural boundaries of the rolled sheet [33-35]. Two basis vectors, $\overrightarrow{a_{1}}$ and $\overrightarrow{a_{2}}$, are defined in the graphene plane and are equivalent by transformation to the unit cell basis. The principal difference being that they connect atomic sites within the unit cell.

Since the boundary conditions of the rolled sheet require that equivalent lattice points are connected, the graphene sheet can be expressed as integer multiples of the two basis vectors (Figure 4). And further, due to the mirror symmetry between the 


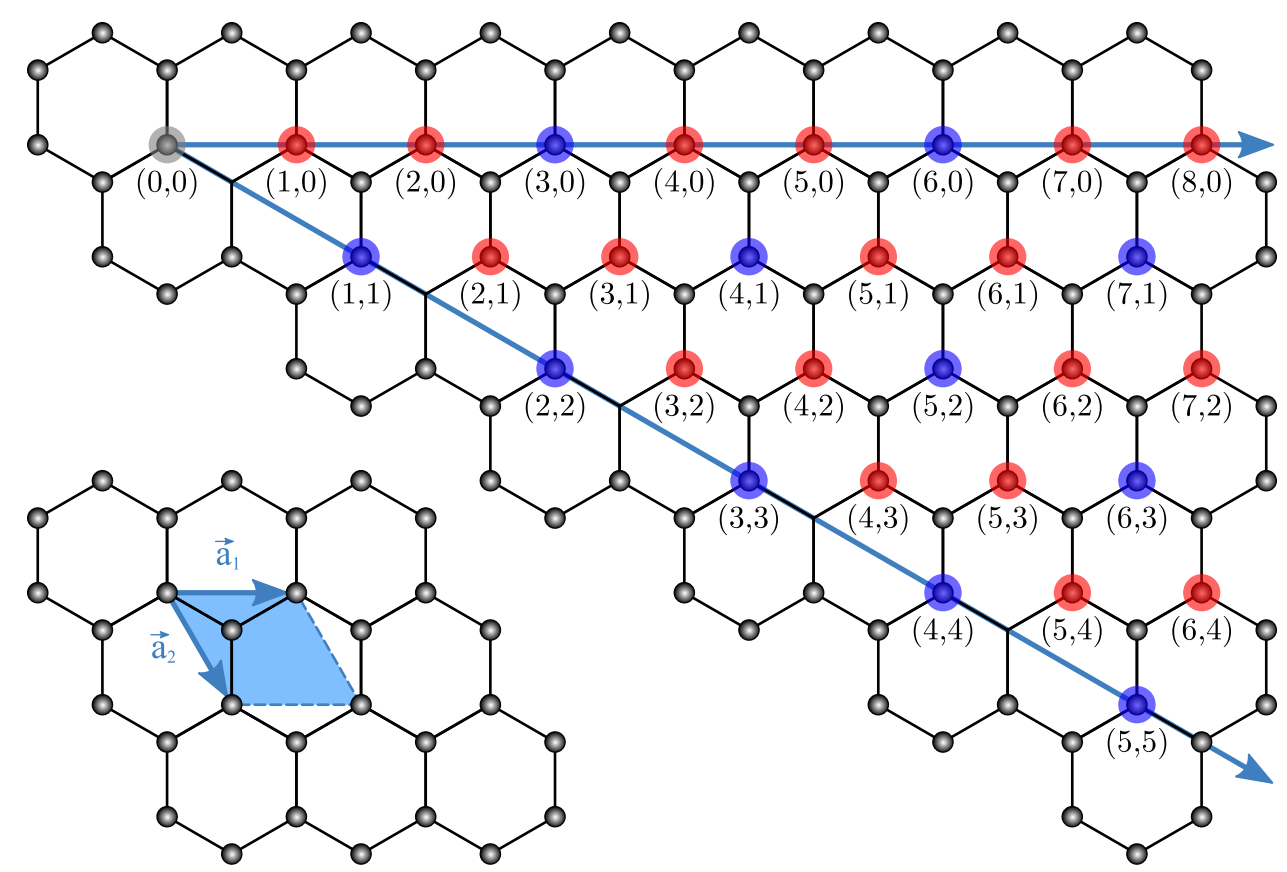

Figure 4: Schematic of 2D basis for CNTs with chirality boundary points labeled. Red indicates semiconducting chiralities, and blue metallic.

two vectors, all possible CNTs can be described by the set of boundary conditions enclosed in a $30^{\circ}$ angle between the two basis vectors. The chiral vector, $\overrightarrow{c_{h}}$, is thereby defined as

$$
\overrightarrow{c_{h}}=n_{1} \overrightarrow{a_{1}}+n_{2} \overrightarrow{a_{2}}
$$

With the chirality of a CNT most commonly expressed using $\left(n_{1}, n_{2}\right)$ notation. Since the chiral vector also defines the circumference of the CNT, the diameter can be trivially expressed as follows:

$$
d_{t}=c_{h} / \pi
$$

A unit cell translational vector, $\vec{T}$, is also defined (perpendicular to $\overrightarrow{c_{h}}$ ) which 
is parallel to the CNT axis

$$
\begin{gathered}
\vec{T}=\left[\left(2 n_{2}+n_{1}\right) \overrightarrow{a_{1}}-\left(2 n_{1}+n_{2}\right) \overrightarrow{a_{2}}\right] / d_{R} \\
d_{R}= \begin{cases}d & \text { if }\left(n_{1}-n_{2}\right) \text { is not a multiple of } 3 d \\
3 d & \text { if }\left(n_{1}-n_{2}\right) \text { is a multiple of } 3 d\end{cases}
\end{gathered}
$$

where $d$ is the greatest common factor of $\left(n_{1}, n_{2}\right)$. This final condition is required to assure that the plane perpendicular to the terminus of $\vec{T}$ is equivalent to that at its beginning. Figure 5 provides a schematic illustration of a $(4,2)$ CNT as an example.

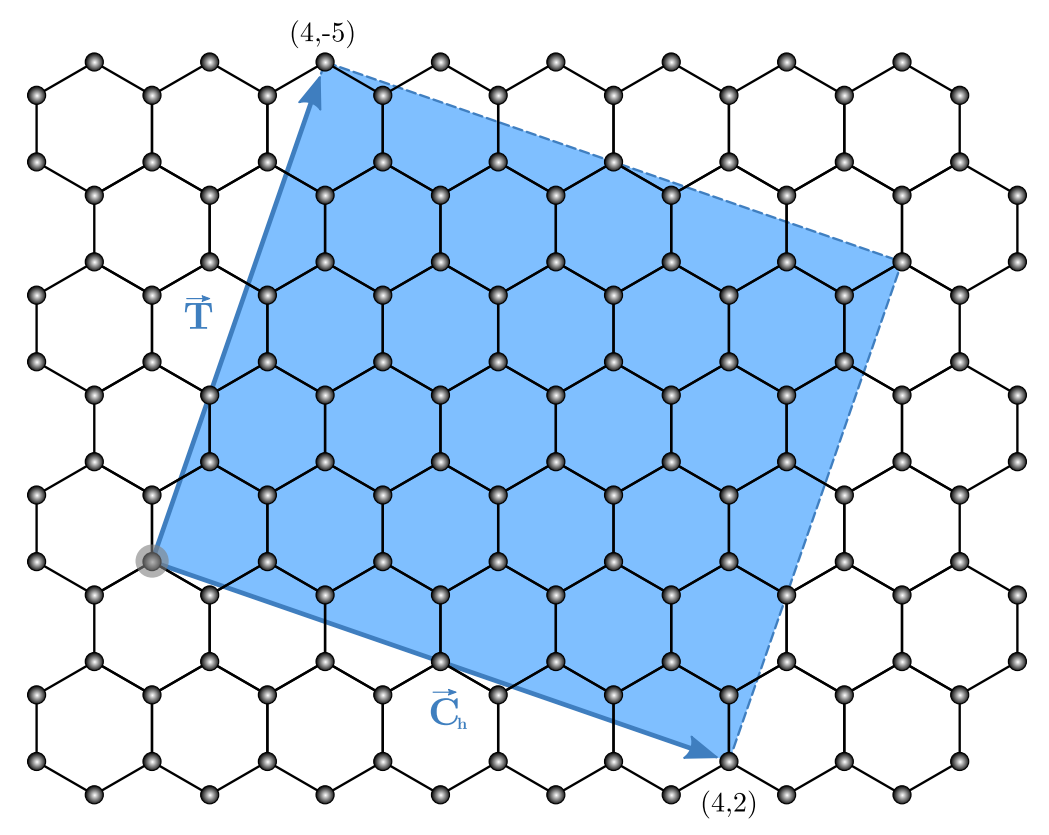

Figure 5: Example unit cell of a $(4,2) \mathrm{CNT}$ with chiral and translational vectors labeled.

While the overall structure of the CNT is composed of a wrapped graphene sheet, the boundary conditions that describe the diameter have profound implications with respect to the electronic structure. Due to this periodic boundary condition, 1D 
(a)

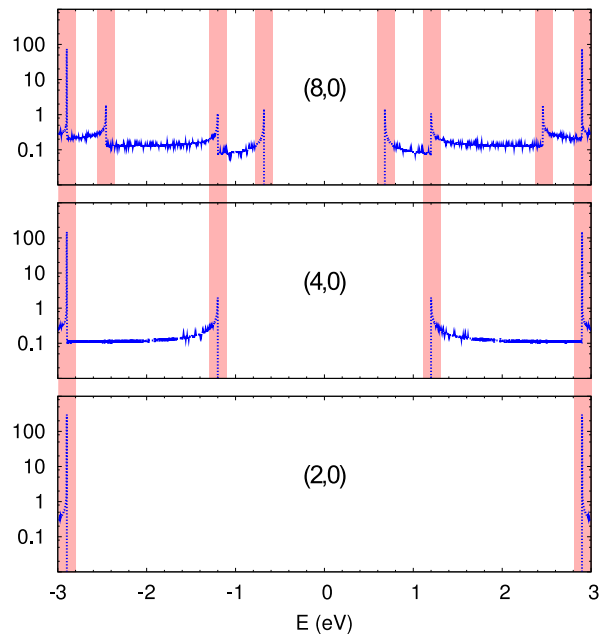

(b)

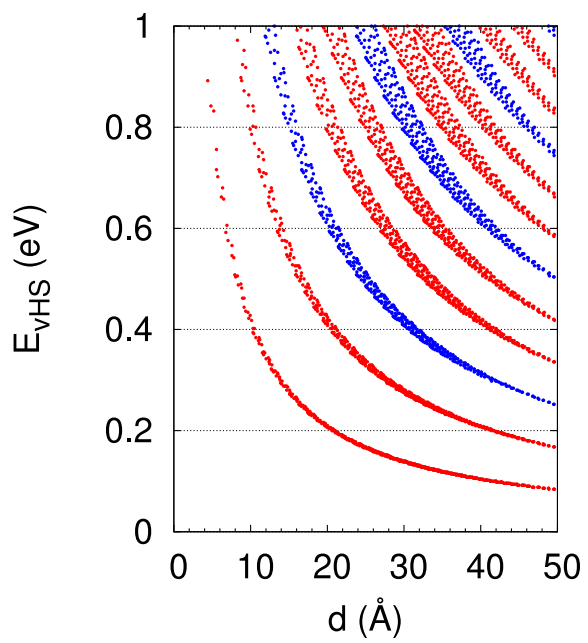

Figure 6: (a) Progression of DoS for $(2,0),(4,0)$, and $(8,0)$ CNTs showing progression of vHSs. (b) Katura plot showing vHS positions as a function of CNT diameter (red: semiconducting, blue: metallic)

energy bands form and correspondingly, van Hove singularities (vHS) in the density of states (DoS) are observed [35]. The nature of these singularities and their relationship to the diameter of the CNT can be further understood by comparing an $\left(n_{1}, n_{2}\right)$ chirality to an $\left(m n_{1}, m n_{2}\right)$ chirality (where $m$ is an integer). That is, a CNTs with an equivalent chiral angle and a diameter which is an integer multiple greater. This is illustrated in Figure 6a.

Note that as the diameter is successively doubled (from $(2,0)$, to $(4,0)$, to $(8,0)$ chirality), so are the number of vHSs in the given energy range. The energy transitions between vHSs are notated as $E_{n n}$ where $n$ is an integer which indexes the vHS position relative to the Fermi level (e.g. the transition from the first band below the Fermi level to the first band above is noted as the $E_{11}$ transition). Further note that each chirality retains the singularities of its chiral factors by necessity of 
the periodic boundary conditions. These singular points can be summarized for all chiralities in so-called "Kataura plots" (Figure 6b) [34]. In addition, there exists a chirality condition for which the density of states is non-zero and continuous near the Fermi energy [33]. Such CNTs are considered metallic in character, lacking the well-defined gap of states between the first vHS above and below the Fermi level observed in semiconducting CNTs. In truth, the electronic characteristics of such CNTs may be referred to as "semimetallic" as they retain drain current saturation and gate-dependence but simply lack a biased "off" state (that is, they are zero band gap semiconductors). The chirality condition for metallic CNTs is as follows (where $m$ is an integer):

$$
2 n_{1}+n_{2}=3 m
$$

Much like single/multilayer graphene, CNTs can grow either single-walled (SW) or multiwalled (MW), depending upon growth conditions. While SW-CNTs can be either semiconducting or metallic in character, MW-CNTs are almost always metallic as their electronic properties are a superposition of the concentric tubes (i.e. with more chiralities contributing towards the overall electronic properties comes a greater chance of any single chirality satisfying the metallic condition). These electronic properties will be central towards our understanding of device performance in CNTs and second-order Raman scattering events. 


\section{Raman scattering in graphitic compounds}

Raman spectroscopy is a technique which can be used to probe the solid state properties of materials. Primarily, this involves the measurement of the frequencies of lattice vibrations (phonons) which are characteristic of specific crystalline structure. This is performed by incident photons either scattering off of phonons or creating phonons and measuring the relative frequency shift (Raman shift) of these photons as they are re-emitted from the sample. In this way, the frequency shift of a Raman peak directly corresponds to the frequency of the phonon generated. The intensity of these peaks are highly dependent upon the polarizability of bonds (and thereby the polarization of incident photons) as opposed to Fourier-transform infrared spectroscopy (FTIR), a similr technique which largely studies polar oscillations and is most widely used for identifying functional groups of materials. These two techniques are thereby complimentary and widely used in materials science and chemistry.

Once again, to understand these phenomena in CNTs, graphene must be first considered. Figure 7 shows the phonon dispersion relation for graphene, indicating the important phonon modes. Modes are annotated with the following abbreviations: i, in-plane; o, out-of-plane; L, longitudinal; T, transverse; A, acoustic; and O, optic (e.g. iLO and oTA refer to the in-plan longitudinal optic and out-of-plane transverse acoustic modes, respectively). While only monolayer graphene is presented, there is little functional variation when additional layers are introduced. Based upon the plane-polarization of the incident radiation, only the 2D Brillouin zone needs to be 
considered [36-38].

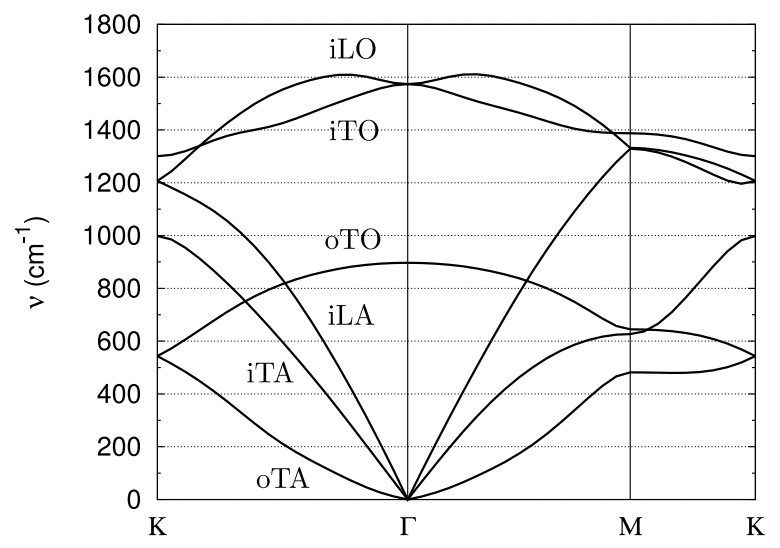

Figure 7: Phonon dispersion relation of monolayer graphene.

There are two peaks of interest which are visible in both graphene and CNTs: the $\mathrm{G}$ and $\mathrm{D}$ peaks. The mechanisms behind these peaks are as follows:

- G: This is a first-order Raman event which involves the scattering of an incident photon off of the degenerate iTO and iLO phonon modes at the Brillioun zone center $(\Gamma$ point). This phonon is characteristic of graphitic carbon compounds and is present in both graphene and CNTs [39].

- D: The D peak is a second order Raman event which arises due to an incident photon first exciting a valence electron into the conduction band $\left(\pi \rightarrow \pi^{*}\right.$ electronic transition). The electron then undergoes inelastic scattering off of an iTO phonon near the K-point, followed by an elastic scattering event off of a defect. A photon is then emitted due to electron-hole recombination. The Raman shift of this photon is equal to the phonon energy of the iTO phonon $[30,37,40-42]$. Since this Raman shift is dependent upon the electron- 
phonon-coupling of an electron first being excited into the condition band, this Raman shift is dependent upon excitation energy. Higher energy photons will result in electronic transitions further from the K-point, and therefore result in scattering off of higher frequency iTO phonons, as per the phonon dispersion relation (Figure 7) [43].

These two peaks can be observed in the example Raman spectrum presented in Figure 8. Note that there is a third peak labeled in this spectrum, the 2D peak (also referred to as the G' peak in rare circumstances). Much like the D peak, the 2D occurs due to a double-resonance process with the exception that the 2D peak does not involve defect scattering, but rather double-scattering with an iTO mode phonon. In graphene, this peak can be used to quantify the number of graphitic carbon layers but the $2 \mathrm{D}$ peak plays no role in the present study.

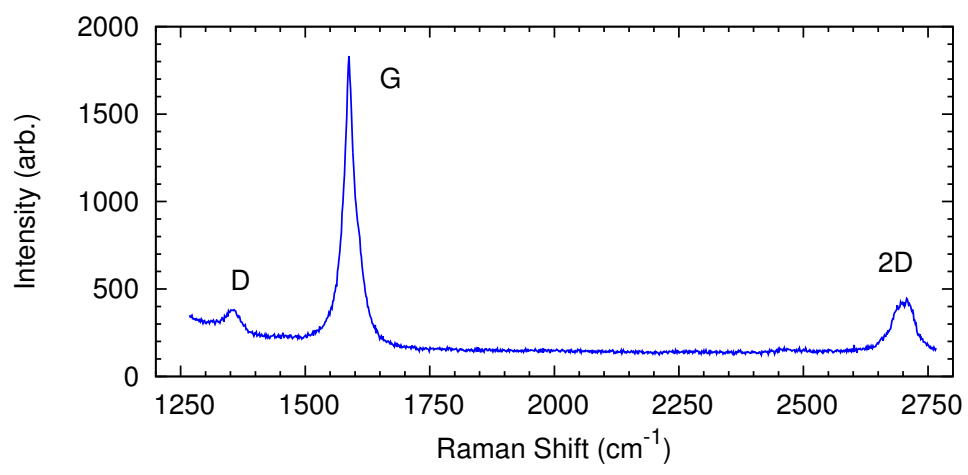

Figure 8: Raman spectrum of a single CNT with D, G, and 2D peaks labeled.

In addition, CNTs can produce a peak which is characteristic of their chirality, called the radial breathing mode (RBM). This peak has some special qualities which arise as a result of the quasi-1D structure of CNTs. As the name suggests, this 
peak results from a oTA phonon mode in which the displacement is radially outward, perpendicular to the CNT axis $[44,45]$. The boundary conditions of this mode cause its frequency to be dependent upon upon the diameter (and thereby the chirality) of the CNT. In addition, the Raman scattering cross-section for the RBM peak is greatly enhanced when an $E_{n n}$ electronic band transition occurs. In fact, in order for the RBM peak to be of measurable intensity, the excitation source of the Raman spectrometer must coinscide within $100 \mathrm{meV}$ of an $E_{n n}$ band transition in the CNT under investigation $[35,44]$. This condition is termed "resonant Raman spectroscopy."

\section{Carbon nanotube field-effect transistors}

In addition to the basic band theory of CNTs, as discussed in Section 2.1, the nature and phenomena observed at metal-semiconductor junctions must be considered in order to have a comprehensive understanding of the CNT-FET operation. The basic design of a CNT-FET is not unlike a typical silicon-based FET and the same fundamental principles govern charge carrier transport. Two metal electrodes (source and drain) are connected by a semiconducting channel (the CNT). A gate electrode spans the channel, insulated from the previous three components, typically by an $\mathrm{SiO}_{2}$ layer. The drain-source bias, $V_{D S}$, provides a potential difference to move charge carriers

across the semiconducting channel. The gate voltage, $V_{G}$, applies an electric field to the channel, distorting its electronic bands, allowing charge carriers to transfer between either electrode and the channel. 
If a metal and semiconductor are brought into contact in this way, reaching thermodynamic equilibrium, the Fermi level of the two materials will match and a Schottky barrier, $\Phi_{S B}$, will form between the free conduction electrons of the metal and the conduction band of the semiconductor. This barrier is the result of a mismatch between the metal-vacuum work function, $\phi_{m}$, and the semiconductor-vacuum electron affinity, $\chi$, according to the following relation:

$$
\Phi_{S B}=\phi_{m}-\chi
$$

Figure 9 schematically illustrates the Schottky barrier phenomenon for arbitrary metal-semiconductor junctions. The barrier height can be modulated by variation in the work function, or by doping of the semiconductor. Junctions with incredibly small barriers are termed "Ohmic contacts" due to their linear drain-source current $\left(I_{D S}\right)$ characteristics. Under this model, known as the Schottky-Mott model $[46,47]$, the electrode work function and semiconductor electron affinity determine a great deal about the device characteristics.

The Schottky-Mott model of metal-semiconductor junctions is, however, somewhat naive. Since the electron wave function must be continuous across the junction, there is a finite penetration of states from the metal into the semiconductor surface (often referred to as metal-induced gap states, due to their residence in the semiconductor band gap). Similarly, surface defects in the semiconductor at the interface can act as electron traps, increasing the electron concentration. These effects can cause 
(a)

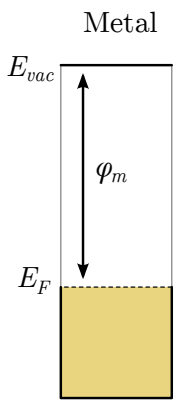

Semiconductor

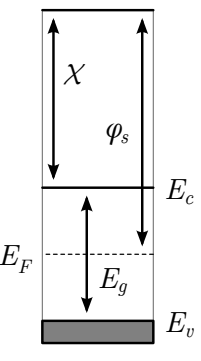

Intrinsic

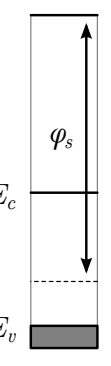

$p$-type

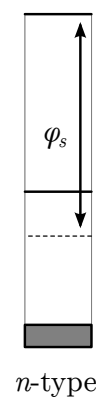

(b)

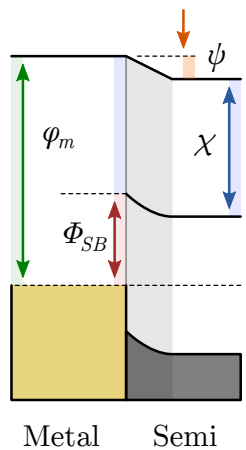

(c)

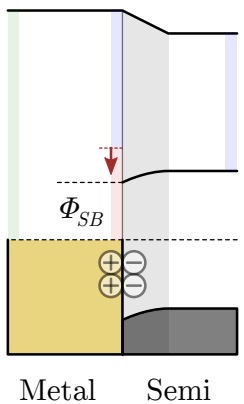

Figure 9: (a) Band diagrams of metals and semiconductors and their junctions (b) according to the Schottky-Mott model and (c) with potential Fermi level pinning effects.

the Fermi level to be deviate from the position which would otherwise be expected by the Schottky-Mott rule (as illustrated by example in Figure 9c). In many cases this can cause the Fermi level to be pulled toward the band gap center regardless as to the difference in work function, an effect known as "Fermi level pinning."

(a)

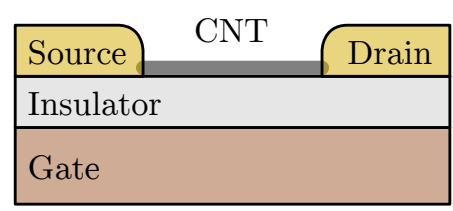

(b)

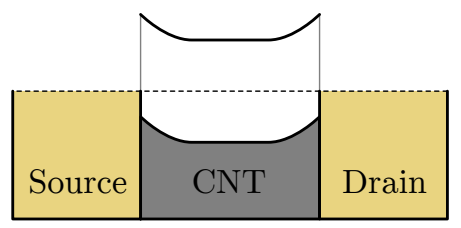

Figure 10: Basic (a) physical architecture and (b) band structure schemes of a CNT-FET device.

A complete CNT-FET device can be fabricated by forming conduction channel interfaces with source and drain electrodes alongside an insulator-isolated gate electrode (Figure 10a) which can be used to distort the CNT channel bands (Figure 10b), permitting the transfer of charge carriers across the source and drain interfaces. This type of device is known as a Schottky barrier FET, modeled as two back-to-back Schottky diodes. 


\section{Charge carrier transport}

Having established the fundamental principles of metal-semiconductor junctions, the mechanisms of charge transport across these junctions can be explored. While FETs are often categorically stated as being in "on" or "off" states, it is necessary to consider a range of operating conditions which showcase the different transport mechanisms.

Without a drain-source bias $\left(V_{D S}\right)$, and thereby no applied field, charge carriers will not cross the source and drain junctions (Figure 11a). Though, even for non-zero $V_{D S}$ (Figure 11b), charge carriers may not be sufficiently energetic to traverse the energy barrier at the metal-semiconductor junction (whether it be the barrier from the metal Fermi level to the semiconductor valence band for holes, or the semiconductor conduction band for electrons). Note that FETs with a metallic CNT channel will always exhibit some drain-source current $\left(I_{D S}\right)$ when biased due to their band of energy states at the Fermi level.

Once a sufficient gate voltage, $V_{G}$, is applied, the resulting electric field perturbs the energy bands of the CNT channel and reduces the effective potential barrier allowing charge carriers to transit the junction. In the earliest stages of transport, energetic charge carriers simply transit over the Schottky barrier, a process known as thermionic emission. As presented in Figure 11c, excited holes overcome the energy barrier at the source electrode junction, and are accepted into valence band states of the CNT. The thermionic emission obeys an exponential relationship relative to the applied bias wherein the current density, $J$, is governed by the following model 
(a)

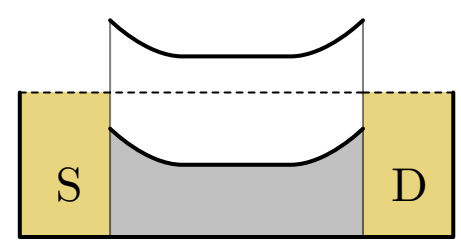

(c)

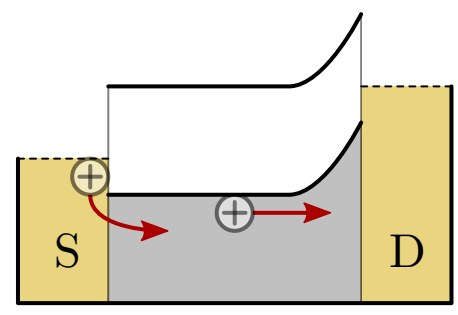

(b)

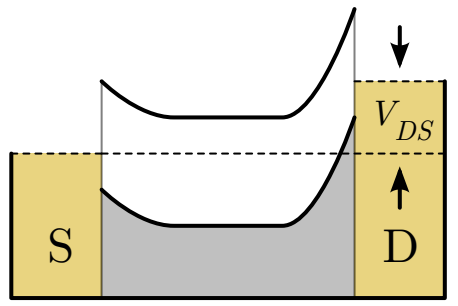

(d)

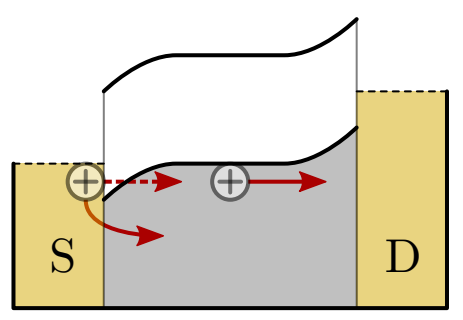

Figure 11: Example schematic of hole injection in a Schottky barrier FET under various operating conditions (a) unbiased, off state, (b) biased, off state, (c) biased, threshold state with thermionic emission, (d) biased, post-threshold/on state with thermionic and field emission. Source and drain electrodes are designated by "S" and "D," respectively.

(where $A_{G}$ and $k_{B}$ are the Richardson and Boltzmann constants, respectively):

$$
\begin{gathered}
J=J_{S}\left(e^{\frac{q V_{D S}}{k_{B} T}}-1\right) \\
J_{S}=A_{G} T^{2} e^{\frac{-q \Phi_{S B}}{k_{B} T}}
\end{gathered}
$$

As the gate field is further increased, the energy bands of the channel further distort, reducing the physical depth of the Schottky barrier, allowing quantum mechanical tunneling of these charge carriers through the barrier (in addition to any thermionic emission), as illustrated in Figure 11d. This phenomenon, known as field emission, becomes the dominant transport model once the magnitude of $V_{G}$ is sufficient to cause the semiconductor band occupied by the charge carriers (conduction 
and valence for electrons and holes, respectively) to cross the Fermi level of the donating electrode. As previously mentioned, the thermionic and field emission transport mechanisms can occur simultaneously in the channel and numerical analysis utilizing these models can be simplified by assesing a regime in which a single mechanism dominates charge transport. These regimes are illustrated approximately in Figure 12.

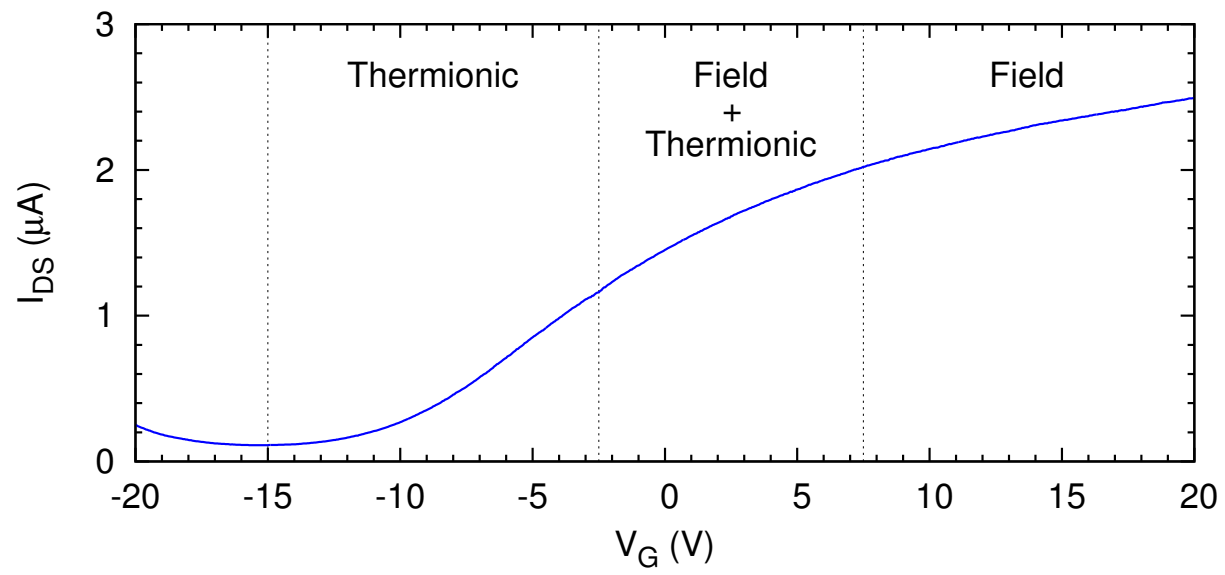

Figure 12: Gate-source characteristics of a single CNT showing majority electron charge transport regimes. 


\section{Experimental Methods}

\section{Quantitative Raman spectroscopy}

A variety of methods can be employed to derive quantitative information from Raman spectra of CNTs and graphene. The analysis most pertinent to this study involves the quantification of defects. Since the Raman D peak involves scattering off of a defect, we can weight the intensity of the D peak relative to the intensity of the $G$ peak (a reference intensity which is not dependent upon an electronic transition) in order to quantify the relative number of defects in the graphitic carbon lattice $[43,48]$. In the case of graphene, lattice boundaries with dangling $p_{x y}$ orbitals constitute defects, thereby necessitating additional controls for flake size. This effect does not play a significant role in defect quantification for CNTs, however.

In the existing literature, the $\mathrm{D} / \mathrm{G}$ ratio has been paired with scanning tunneling microscopy to compute an empirical relationship between the $\mathrm{D} / \mathrm{G}$ ratio and absolute defect densities $[31,49,50]$. In these studies, graphene samples were also subjected to ion bombardment to introduce controlled numbers of defects, producing a gradient of defect densities. This allowed for defect density regimes to be more rigorously defined and allowed for more accurate use of the $\mathrm{D} / \mathrm{G}$ ratio as a comparative measurement. This type of measurement will be of primary interest for this study.

The primary points of interest in this study are that: (1) the absolute number

of defects can be quantified directly from the D/G ratio, (2) the relationship between 
defect density and $\mathrm{D} / \mathrm{G}$ ratio is non-linear and, for sufficiently large ratios, becomes non-monotonic, (3) the wavelength of the excitation source must be taken into account (which was, up until that point, expected as a matter of course), and (4) that other spectral features (which include peak width) may be helpful in identifing regimes of defect density. While prior studies have used the $\mathrm{D} / \mathrm{G}$ ratio as a quantification metric, this was the first comprehensive effort to express the $\mathrm{D} / \mathrm{G}$ ratio progression and validates its current use. These absolute quantification models will be applied and discussed in Section 7.1.

In addition to defect quantification, Raman spectroscopy can be used to determine the chirality of an individual CNT if an RBM peak is observed. To do so, one must first note the $E_{n n}$ transitions that correspond with the excitation source energy $\left(E_{\text {laser }}\right)$. Then, the diameter of the CNT can be calculated from the RBM peak frequency using the following forumla [51]:

$$
d_{t}=248 / \omega_{R B M}
$$

where $d_{t}$ is in $\mathrm{nm}$ and $\omega_{R B M}$ is in $\mathrm{cm}^{-1}$. Note that the constant, $248 \mathrm{~cm} \cdot \mathrm{nm}$, is determined empirically and encompasses a variety of more fundamental physical properties (there are other variations of this relationship with additional correction factors). Combining the values for $E_{\text {laser }}$ and $d_{t}$, a single point can be located on the Katura plot (Figure 6b) which corresponds to the $E_{n n}, d_{t}$ combination of a specific chirality. While the chirality of a CNT is the determining factor in its optoelectronic 
properties, the RBM peak is not used for chirality identification in this study. As mentioned, the RBM frequency is dependent upon excitation energy. As the excitation source must be within approximately $100 \mathrm{meV}$ of the vHS separations in the DoS, Raman spectrometers with fixed-wavelength excitation sources are inherently handicapped in their ability to produce and detect RBM phonon scattering.

\section{Polarization-dependent Raman spectroscopy}

In addition to the prevously discussed implication of the quasi-1D structure of CNTs, Raman intensity is strongly correlated with the polarization of the excitation photons with respect to the CNT axis $[24,25]$. In order to evaluate this effect, Raman spectra were collected using a $532 \mathrm{~nm}$ excitation source from single CNTs (further spectrometer details can be found in Section 4.1).

A half-wave plate was used to rotate the polarization of the beam one complete revolution, collecting spectra at $10^{\circ}$ polarization intervals. Figure 13 a depicts the variation in G peak intensity for a single CNT subjected to these incident polarizations. An alternative method to incrementally rotating polarization is continuously rotating polarization $(\mathrm{CRP})$ of the excitation source. By rotating the wave plate through an integer multiple of quarter revolutions during the acquisition time (that is, an integer number of $180^{\circ}$ changes in polarization), it is possible to collect an averaged spectrum which removes polarization-dependent effects from the observed

spectrum. The convenience of this result comes at the expense of measured intensity 
relative to measurements with the incident polarization parallel to the CNT axis, as shown in Figure 13b.

(a)

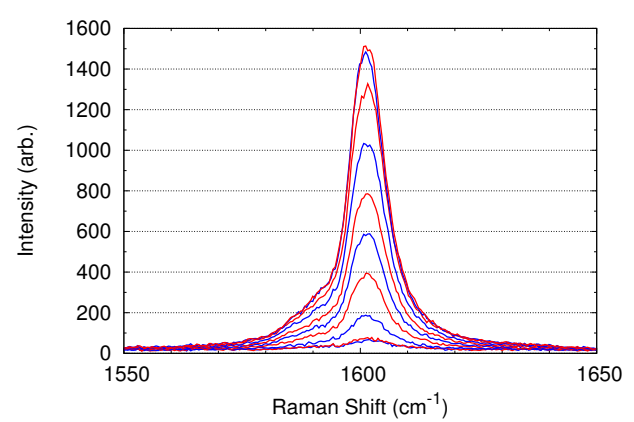

(b)

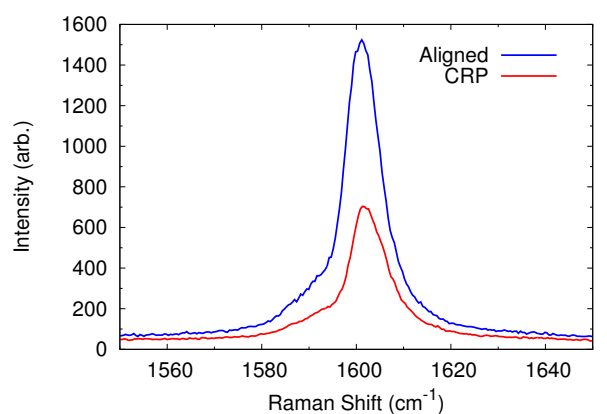

Figure 13: Raman G-band spectrum of a single CNT (a) with polarization vector varied from $0-90^{\circ}$ relative to the CNT axis and (b) relative intensity when collected with aligned polarization and CRP.

The reduction of intensity due to CRP by approximately $1 / 2$ as observed in Figure $13 \mathrm{~b}$ is as expected. Since the Raman scattering event requires incident polarization parallel to the CNT axis, the number of Raman scattering events will be proportional to the cosine of the angle, $\theta$, between them. The measured intensity (i.e. the magnitude of the Poynting vector), which is proportional to the square of the electric field, will thereby follow a $\cos ^{2} \theta$ relationship. If the polarization is rotated continuously for an integer number of revolutions through spectrum acquisition, the time-averaged intensity, $\bar{I}$, is given by:

$$
\bar{I}=\frac{1}{t_{a c q}} \int_{0}^{t_{a c q}} I_{0} \cos ^{2}\left(\frac{m \pi t}{t_{a c q}}\right) d t=\frac{I_{0}}{2}
$$

Where $t_{a c q}$ is the acquisition time, $I_{0}$ is the maximum observable intensity (wherein the polarization vector is parallel to the CNT axis, or $\theta=0$ ), and $m$ is an inte- 
ger. This relationship is further validated by plotting the intensity of the $\mathrm{D}$ and $\mathrm{G}$ peaks (Figure 14a) with respect to $\theta$, which shows a strong correlation with a $\cos ^{2} \theta$ relationship.

(a)

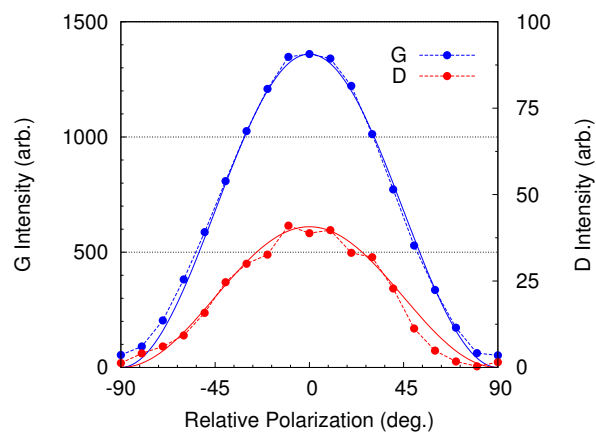

(b)

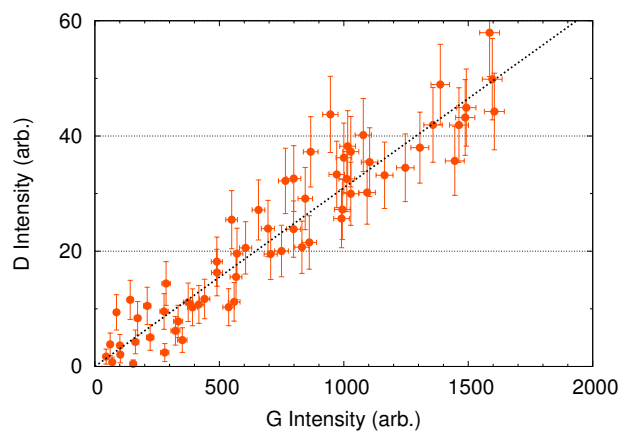

Figure 14: Raman D and G peak intensities of a single CNT plotted with respect to (a) polarization angle, compared against a $\cos ^{2} \theta$ trendline, and (b) to each other showing that the $\mathrm{D} / \mathrm{G}$ ratio is linear and independent of polarization.

Plotting the measured D peak intensity against $\theta$ further validates the $\cos ^{2} \theta$ relationship (Figure 14a). Noting this, the D and G intensities scale linearly with one another, as shown in Figure 14b. That is, the D/G ratio is independent of incident polarization and can be effectively computed so long as the polarization conditions yield sufficient spectrum intensity. This in turn validates the use of the $\mathrm{D} / \mathrm{G}$ ratio as a quantification metric with CRP Raman spectrum acquisition. While these conclusions may seem ancillary in the context of single point spectra (especially where use of a half-wave plate is feasible to manipulate the incident polarization), they are of central importance for Raman mapping, and defect quantification, of CNT-FETs.

Such methods to remove polarization-dependence must be developed for the sake of spatially mapping spectrum variations in CNTs. Growth of CNTs will, in the 
(a)

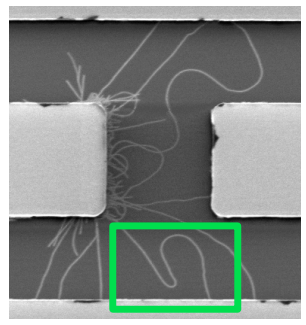

(b)

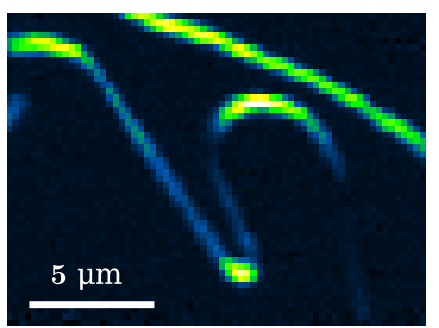

(c)

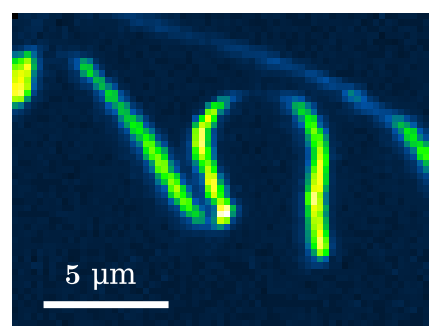

Figure 15: (a) SEM image of a CNT-FET with region of interest indicated. Corresponding Raman spectrum maps of the RBM peak intensity using (b) a horizontally and (c) vertically polarized source

absence of extensive external controls, occur following a completely arbitrary path. If a fixed polarization is used to acquire a spectrum map, the intensity of various peaks will be more indicative of the relative polarization than actual scattering effects in the material. This effect is illustrated in Figure 15, wherein the RBM intensity of a single, highly contoured, CNT was mapped. The CRP spectrum technique is therefore of great interest, especially with regards to defect mapping. After all, mapping with fixed polarization would require the acquisition of a redundant map to acquire signal from regions where the CNT path was perpendicular to the original polarization, consuming many additional hours of laser lifetime. In addition, great care and consideration would be required when correlating these maps. Beyond stitching errors in locations where the CNT axis drastically changes direction, the impact of variations in signalto-noise ratio within individual maps must be mitigated. 


\section{Electron transport measurements}

Measuerment of the basic gate- and drain-source characteristics of the CNT-FETs can provide a wealth of knowledge about intrinsic properties of the CNT channel. Most notably, from the gate-source characteristics of the FET, it can be directly observed whether the CNT is metallic or semiconducting as shown in Figure 16. Since metallic CNTs have a constant non-zero DoS near the Fermi level (chiralities specified by the condition in Equation 4), a drain-source bias will always allow current to cross the channel regardless of the gate voltage. Some gating characteristics are still observed due to the vHSs and the relatively low DoS in the mid-gap region (which contribute towards the metallic behavior). This is in contrast with the purely semiconducting CNTs which have no metallic characteristics and thereby have higher on/off ratios. From these overt differences it is possible to discern whether the CNT falls into the metallic or semiconducting subset of chiralities.

(a)

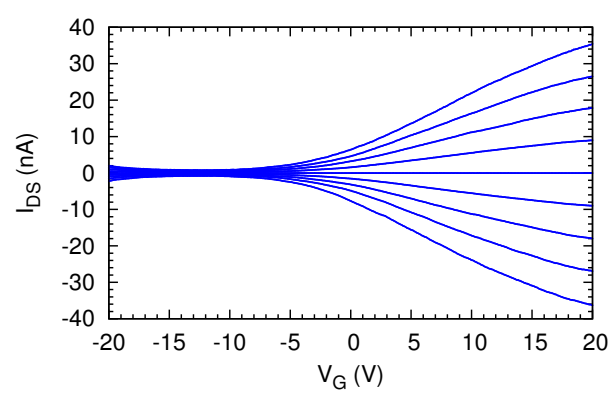

(b)

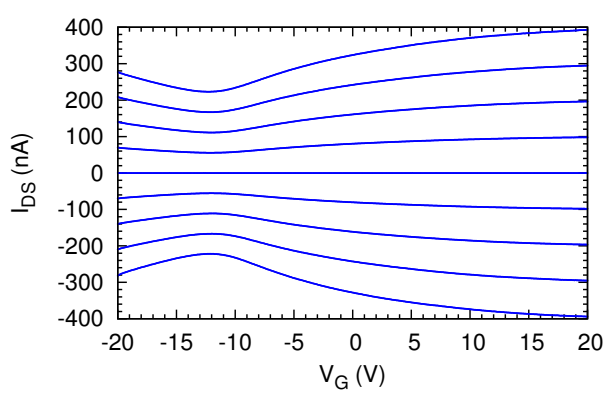

Figure 16: Gate-source characteristics of (a) semiconducting and (b) metallic CNTs with $V_{D S}$ varied from $-1 \mathrm{~V}$ to $1 \mathrm{~V}$ in $250 \mathrm{mV}$ increments. 


\section{Electrical impact of adsorbates}

Previously, it was introduced that the electron transport properties of CNT-FETs are dependent upon the composition of the ambient atmosphere. One would indeed hypothesize this to be the case for a transport channel which is composed entirely of surface atoms. In order to measure the intrinsic properties of the CNT-FET (that is, absent surface-adsorbed gas molecules which impact the device characteristics), a probe station must include a chamber pumped down to ultra-high vacuum (UHV) conditions. In the process, the sample and chamber surface are subject to a desorption process (usually a bake-out) to drive off the weakly-adsorbed surface molecules. Note that bake-out temperatures are far lower than what would be required to cause morphological changes to the CNT-FETs. After the temperature has stabilized and the chamber pressure is sufficiently low, the intrinsic characteristics can then be measured. To measure the response of a particular gas species, controlled quantities of the gas must be introduced into the chamber in order to reach a desired working pressure for additional measurements. Such a comparison is presented in Figure 17, wherein the gate-source characteristics of a single CNT-FET were measured under various pressure conditions (as indicated in the figure).

Immediately visible in these data is the shift in the CNT-FET "off" state (and thereby the threshold gate voltages). Two principal mechanisms have been offered in the current literature to account for this threshold voltage shift, revolving around modulation of the Schottky barrier formed at the metal-CNT interface and doping of 
(a)

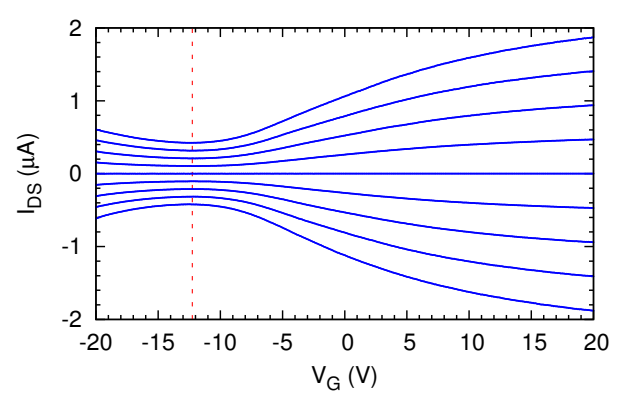

(c)

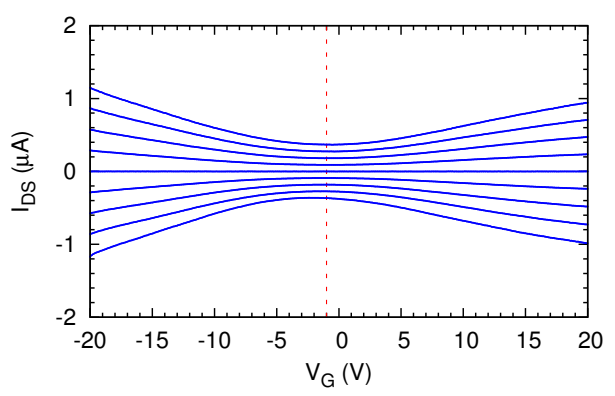

(b)

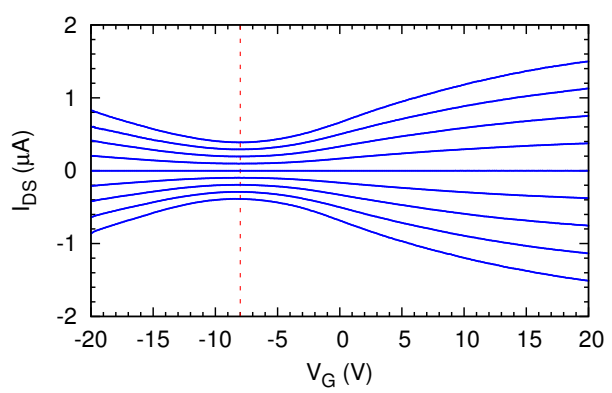

(d)

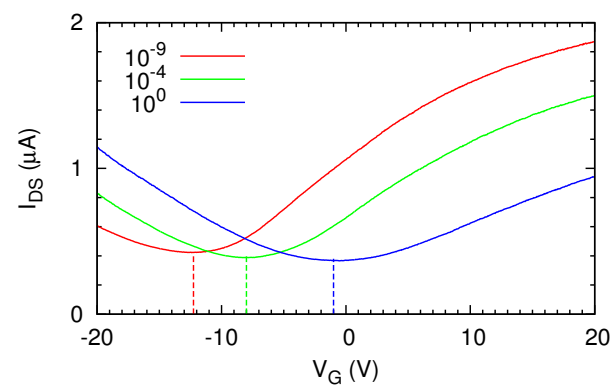

Figure 17: Gate sweeps of a single CNT with $V_{D S}$ varied from $-1 \mathrm{~V}$ to $1 \mathrm{~V}$ in $250 \mathrm{mV}$ increments at approximately (a) $10^{-9}$, (b) $10^{-4}$, and (c) $10^{0}$ Torr chamber pressure. (d) Single gate sweeps at each pressure setting shown for comparison $\left(V_{D S}=1 \mathrm{~V}\right)$. Dashed lines indicate locations of $I_{D S}$ minima.

the CNT channel.

The first mechanism suggests that the shift in threshold voltage is due to a change in contact potential (and thereby, the work function) of the electrode materials due to the adsorption of gases to the surface. While it is well known that the work function of metals can be modulated by annealing in a forming gas (indeed, this is a well utilized procedure in semiconductor manufacturing facilities to assist with barrier tuning), work functions have also been shown to change in the presence of various gas species [52]. Since it is possible for CNTs to have very narrow band gaps (many on the order of $0.25 \mathrm{eV}$ ), even slight changes in work function could result in a large 
impact on the threshold voltage.

An example of this mechanism is depicted schematically in Figure 18b, where an increase in work function results in an increased Schottky barrier for electrons at the CNT-electrode junction (and a corresponding decrease in the barrier for hole injection). Recalling the charge transport mechanisms discussed in Section 2.4 (specifically as represented in Figure 11), it is clear that these effects will impact the amount of drain current measured with a given gate and drain bias, manifesting as a threshold voltage shift.

(a)

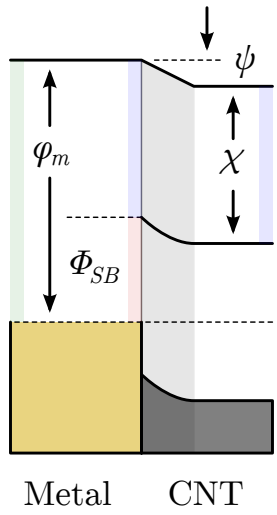

(b)

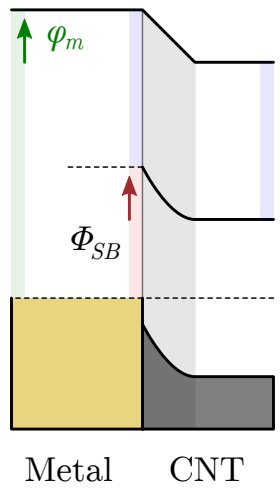

(c)

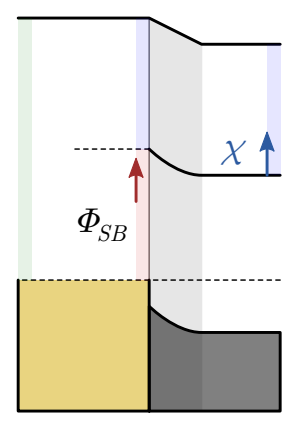

Metal CNT

Figure 18: (a) Intrinsic CNT-electrode junction with relevant quantities labeled. Increases in Schottky barrier height caused by (b) a change in the electrode work function and (c) a change in CNT electron affinity.

The principal mechanism reported in the literature offers that the ambient $\mathrm{O}_{2}$ molecules attach to defect sites on the surface of the CNT, acting as p-type dopants or traps for negative charge carriers. These charge traps result in a downward shift of the Fermi level in the CNT due to decreased electron occupancy, which in turn modulates the Schottky barrier of the junction, as illustrated in Figure 18c [53-57]. 
While both of these mechanisms fundamentally revolve around the Schottky barrier height, the second mechanism, which is more widely accepted, has some additional caveats which make it particularly ripe for consideration in the present study. Much of the present literature postulates that greater concentrations of CNT surface defects will result in increased surface adsorption of ambient gases which should, in turn, increase the magnitude of threshold voltage shifts observed in the gate-source curves at varied oxygen concentrations [57-59].

By applying the previously described quantitative Raman methods in conjunction with the gate-source characteristics, it will be possible to provide a correlation between defect concentrations in the CNT channel to the threshold voltage shifts, and thereby validate the adsorbate doping model for CNT gas sensing. 


\section{Instrumentation Developments}

In order to discern whether or not CNT lattice defects play a role in adsorption of ambient oxygen, and thereby influence threshold voltage in the CNT-FET device, there are two principal requirements of measurement and testing instrumentation: (1) a Raman spectrometer with a high-resolution stage capable of spatial mapping and manipulation of incident beam polarization, and (2) a probe station which can perform the necessary 3-probe measurements while also controlling and characterizing trace-level ambient gases. These requirements initiated several instrumentation developments for polarization control in a commercial Raman spectrometer and the construction of a custom ultra-high vacuum probe station system.

\section{Raman spectrometer}

Raman spectra for this study were collected using a Horiba LabRAM HR800 Raman/photoluminescence spectrometer. This system is equipped with a $532 \mathrm{~nm}, 100$ $\mathrm{mW}$ diode-pumped solid state laser, and a $325 \mathrm{~nm}, 15 \mathrm{~mW} \mathrm{HeCd}$ gas laser. Of the objective lenses available on the instrument, a 50x 0.55 NA lens was used for mapping and characterization. For mapping functionality, two stages are attached: a large-range motorized stage and a piezoelectric XY-scan stage capable of $50 \mathrm{~nm}$ step intervals over a range of $\pm 200 \mu \mathrm{m}$ relative to the equilibrium position. The optical pathway of the system is depicted in Figure 19. The clean-up filters at the entrance have a $2 \mathrm{~nm}$ spectral width, and the dichroic cut-off filters at the objective-end have 
reflection/transmission edges steeper than $100 \mathrm{~cm}^{-1}$.

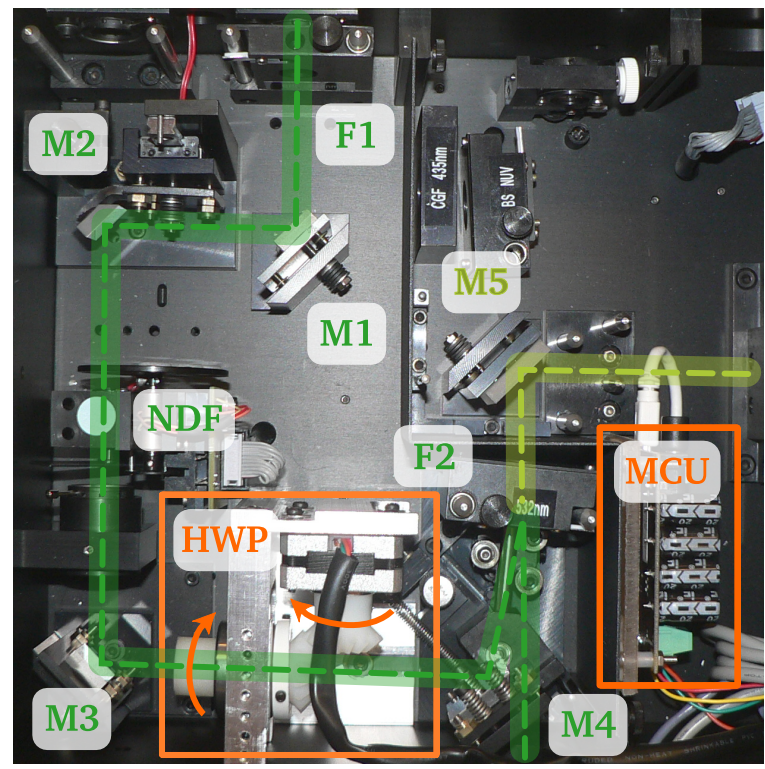

Figure 19: Optical pathway of the HR800 system with various optical components labeled: filters (F1, F2), mirrors (M1-M5), neutral-density filter wheel (NDF), half-wave plate mount (HWP), and motor control unit (MCU)

\subsubsection{Motorized half-wave plate}

The motorized half-wave plate (HWP) system consists of a $532 \mathrm{~nm}$ half-wave plate inserted into a custom mounting block which is engaged by a stepper motor via a pair of miter gears. Rotation of the HWP was stabilized with a roller bearing sleeve (Figure 20a). The stepper motor allows the HWP to be rotated in minimum steps of $1.8^{\circ}$, which corresponds to a $3.6^{\circ}$ step in polarization angle. If desired, this could be reduced further by introducing mechanical advantage in the miter gears. A custom software application (Figure 20b) was written to control the HWP during spectrum acquisition. This application mimics all operations of the manufacturer- 
supplied control software: neutral density filter (NDF) selection, CCD camera image acquisition, spectrum collection parameter control (acquisition time, accumulations, grating selection, etc), and stage control for mapping functionality. The software adds the HWP control, allowing two principle modes of acquisition: (1) the previously mentioned CRP technique, and (2) incremental polarization rotation for polarizationdependent point spectra. In addition to the schematics and screenshots offered in Figure 20, the mounting bracket can be observed top-down in the photograph in Figure 19.

(a)

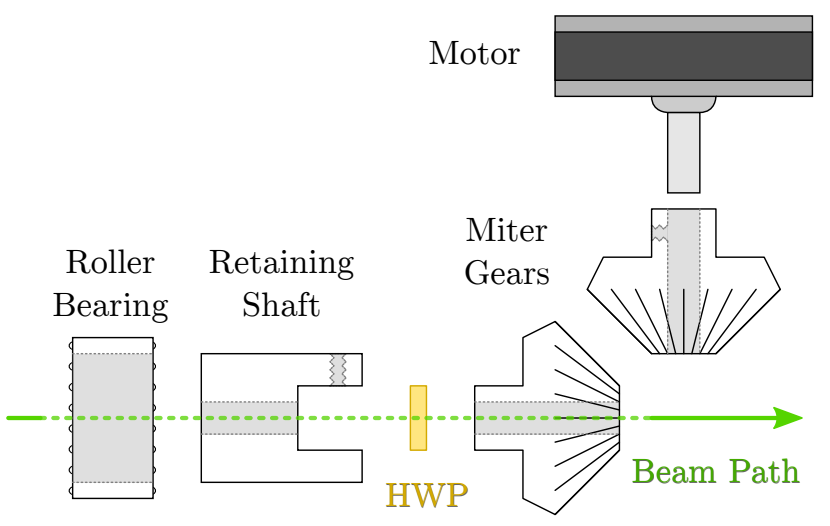

(b)

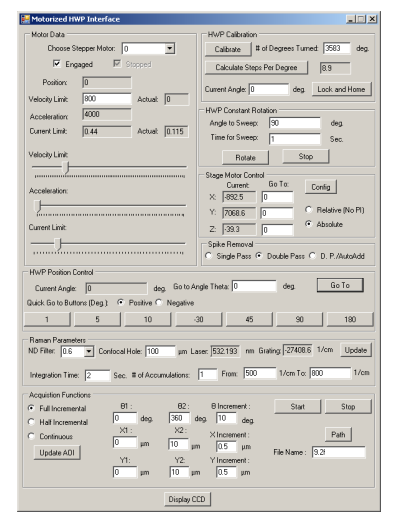

Figure 20: (a) Exploded diagram of motorized HWP system (photograph visible in Figure 19). (b) Software control interface (live CCD imaging window not shown).

\subsubsection{Quarter-wave plate optics}

In addition to the motorized HWP system, a quick-change mount (used in place of the HWP mount) was fabricated to allow 3 optical components to be slid into and out of the beam path (Figure 21). Using this mount, a quarter-wave plate (QWP) could be inserted and used to circularly polarize the Raman source in order to achieve the 
same general effect of the CRP technique, removing polarization-dependence from Raman spectrum maps. The QWP was used in this fashion to collect the maps used for defect quantification.

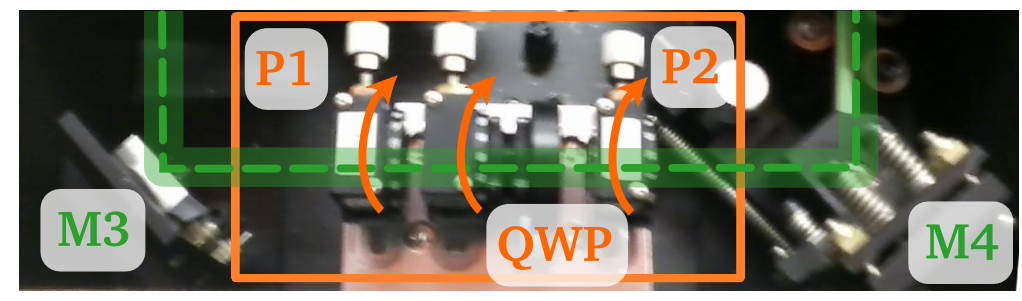

Figure 21: Optical pathway of the HR800 system with QWP mount inserted showing the cleanup polarizer, P1, and the calibration polarizer, P2, which is not utilized during measurements.

By inserting linear polarizers in the slots preceding and following the QWP, it was possible, using a power meter, to calibrate the angle of the QWP to produce circular polarization. Aligning the clean-up polarizer, P1 with the intensity maximum of the source removes ellipticity, while the calibration polarizer, P2, is positioned to extinguish the beam (with its polarization axis oriented perpendicular to P1). Inserting the QWP between P1 and P2, and orienting it such that the exit beam (after P2) is of maximum intensity assures that the light exiting the QWP is circularly polarized, meaning that the polarization of the incident photons (from P1) are at a $45^{\circ}$ angle with respect to the fast/slow axis of the QWP. This can be further validated by rotating $\mathrm{P} 2$ through $180^{\circ}$ and observing no change in intensity. The calibration polarizer, P2, is then removed before conducting measurements.

Confidence in well maintained polarization through the spectrometer optics is provided by observing the strong $\cos ^{2} \theta$ relationship in Figure 14 and the expected $50 \%$ 
intensity reduction when using the CRP technique (Figure 13). These two results, as presented in Section 3.2 would not be observed without a well maintained polarization state.

\section{Micro-environmental probe station}

In order to measure the unique electronic response of CNTs to various environmental stimuli, the micro-environmental probe station (MEPS) system was designed and constructed. It consistes of an ion-pumped vacuum system that is capable of achieving a base pressure of $3 \times 10^{-9}$ Torr which is attached to a mini-chamber with 8 additional accessory ports. The ports are loaded with the following: three high-voltage coaxial feed-through probe arms (two of which equipped with software-controlled motors), one fiber armature, a variable gas leak valve with up to $1 \times 10^{-10}$ Torr $\cdot \mathrm{L} / \mathrm{s}$ flow rate control, a 4-channel SRS residual gas analyzer (RGA) with an $0.1 \mathrm{ppb}$ measurement floor, vacuum gauges, and a heated/cryogenic stage capable of maintaining sample temperatures from 10-500 K. Additional accessories consist of an Agilent B1500A semiconductor parameter analyzer, Kiethly high-voltage power supply $(2 \mathrm{kV})$, and a DelMar Trestles Ti:Sapphire tunable infrared (IR) laser (700-950 nm, 0-350 mW). A labeled chamber overview is depicted in Figure 22. 


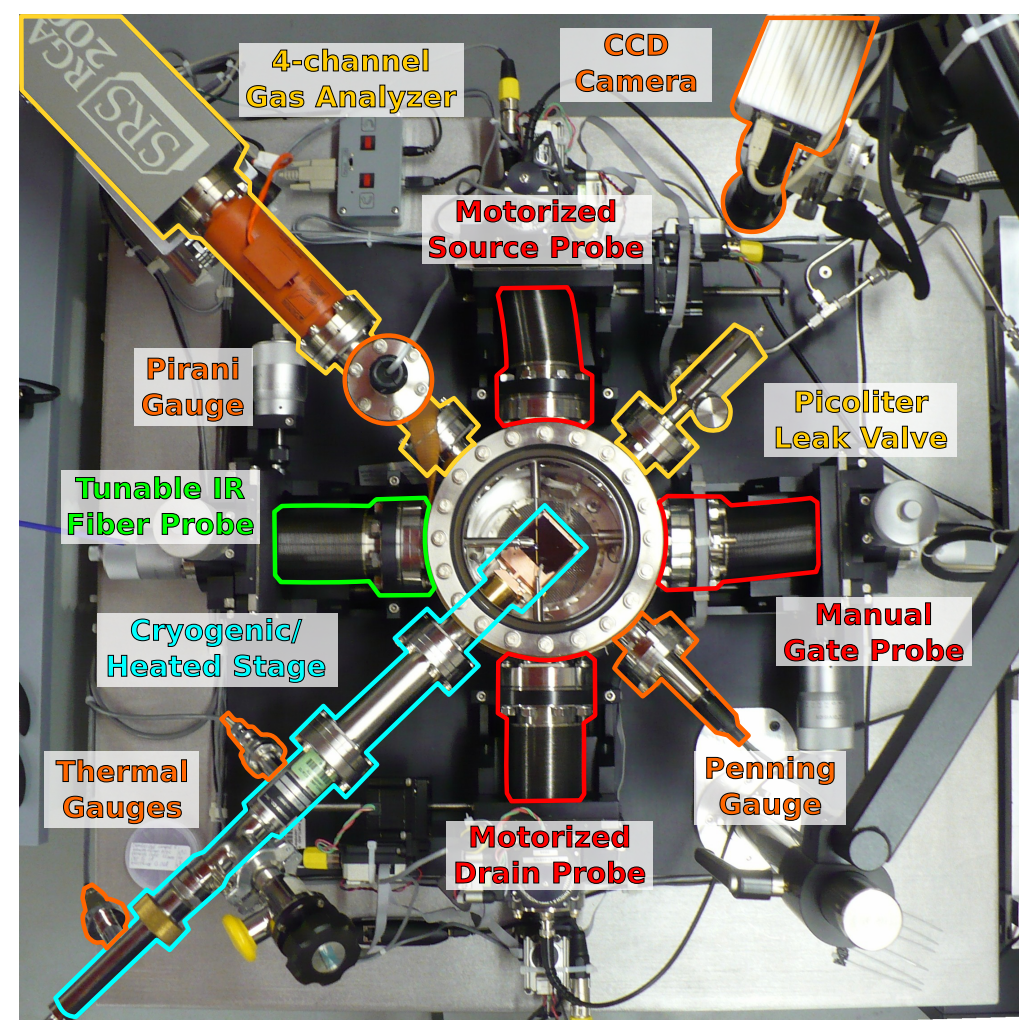

Figure 22: MEPS chamber overview with accessories labeled.

\subsubsection{Gas injection and sensing}

The principle goal of the MEPS was to enable the measurement the electronic response of CNT-FETs to ambient gases. The first requirement for such a study is to drive-off adsorbates from the surface of the CNT. This includes a bake-out and ultraviolet (UV) photodesorption process: ramping of the vacuum system to at least $5 \times 10^{-6}$ Torr (by operating rotary and turbomolecular pumps), followed by an $18 \mathrm{hr}$ wholechamber and sample bake-out at $200{ }^{\circ} \mathrm{C}$. After allowing the chamber to rest at thermal equilibrium for approximately $12 \mathrm{hrs}$, an UV lamp (15 W at $250 \mathrm{~nm}$ ) is then use for $1 \mathrm{hr}$ to induce photodesorption. The benefit of the photodesorption process is two- 
fold: higher vacuum levels can be achieved, and molecules with higher enthalpies of desorption (such as $\mathrm{H}_{2} \mathrm{O}$ ) can be removed from surfaces. In addition, if the use of an RGA is required, the RGA filament should be ramped and degassed before the bakeout procedure in order to avoid the introduction of additional contaminants into the chamber. Figure 23 shows the benefit of the bake-out and photodesorption process. In absolute terms, the bake-out procedure can reduce the ultimate pressure by more than an order of magnitude but of primary interest is that the partial pressure of $\mathrm{H}_{2} \mathrm{O}$ is reduced by approximately a factor of 2 relative to nitrogen. In addition, $\mathrm{O}_{2}$ levels are reduced to the noise floor of the instrument.

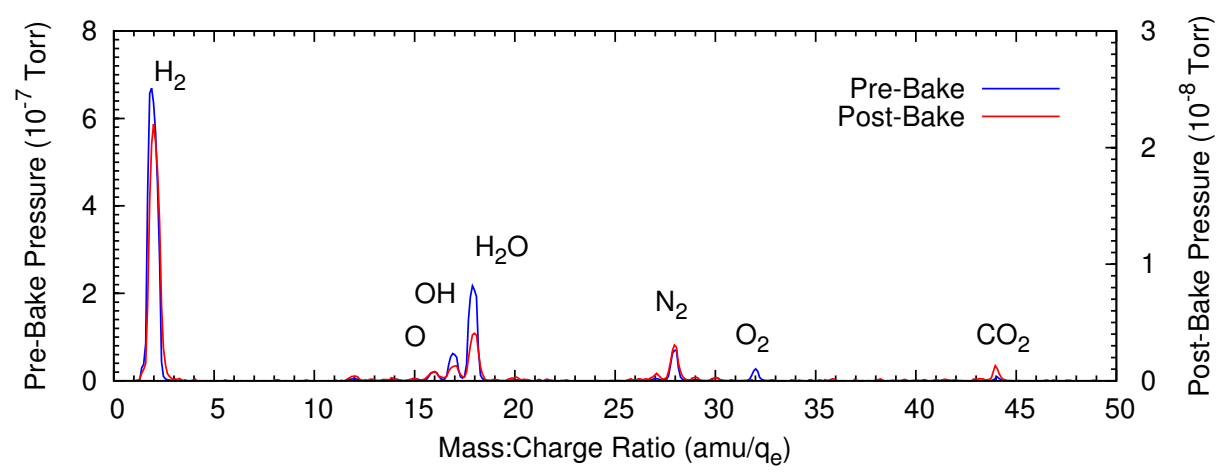

Figure 23: Analog partial pressure histogram scan before and immediately after bake-out and photodesorption process with well known gas species labeled.

The injection of gas species itself was facilitated by the addition of the RGA and the variable leak valve. The precision valve allows an inlet of gases (in this case, $\mathrm{O}_{2}$ was studied exclusively) while pressure and gas concentrations are monitored using the vacuum gauges and RGA, respectively. The combination of the bake-out procedure and gas injection system allow environments to be created with $>95 \%$ 
purity of the target gas species at $5 \times 10^{-6}$ Torr. Figure 24 shows a logarithmic plot of various gas concentrations as a function of time as $\mathrm{O}_{2}$ is injected into the chamber (with the RGA in 4-channel live monitor mode). After evacuating and purging the inlet lines and opening the leak valve at approximately $0.5 \mathrm{hrs}$, the $\mathrm{O}_{2}$ gas concentration increases by a factor of approximately $10^{4}$. Whereas $\mathrm{N}_{2}$ increases by less than a factor of 10 and the increase in water vapor is not sustained after 2 hrs.

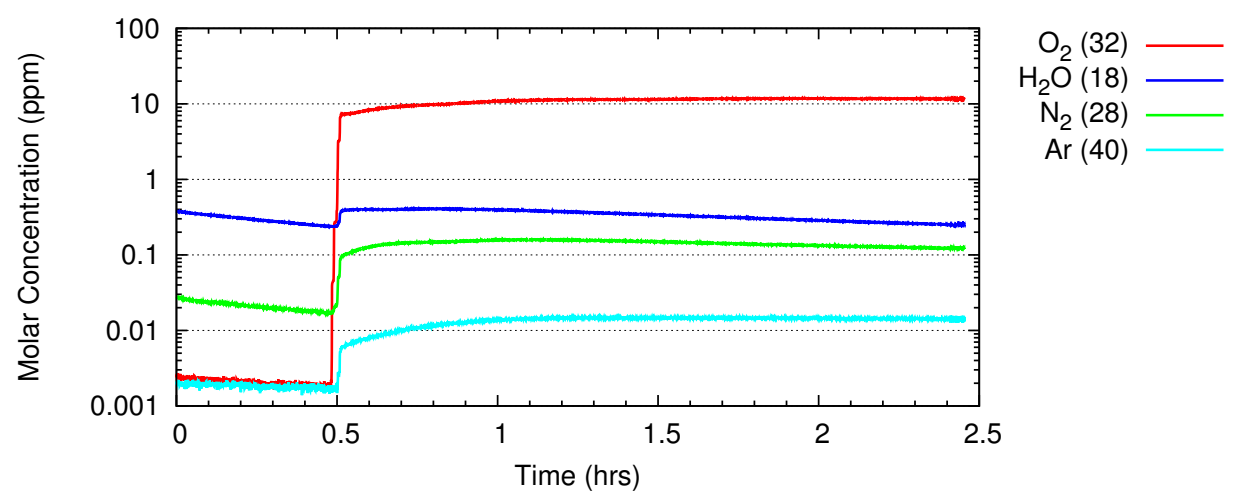

Figure 24: Gas concentration curves as measured by the RGA as $\mathrm{O}_{2}$ is injected into the system at approximately $t=0.5 \mathrm{hrs}$. Mass-charge ratios are indicated in the legend. 


\section{Computational Methods}

While the analysis of individual Raman spectra or drain current curves are mathematically trivial, the scale of this correlative study presents numerous computational challenges. Consider the number of data points subject to consideration: For the electronic characterizations, gate sweeps are each composed of 801 data points $\left(I_{D S}\right.$ vs. $V_{G}$ values) at 9 different $V_{D S}$ settings. These gate sweeps are collected 3 times and averaged for each of 30 devices across 3 different chamber pressures (1,946,430 unique data points). With respect to Raman measurements, each of the 31 analyzed devices contain an average of 2,958 map points, which are composed of 1,024-channel spectra (intensity vs. Raman shift data points) and their associated map coordinates (90,869,760 unique data points).

The principal consideration is that the scale necessitates custom software with a carefully designed data structure that provides a convient system for parsing source data files, looking up specific data points of interest, iteration through a series of data, storage of computed values, and proof-of-concept computations. While these topics are not discussed in further detail, they are noted as an important point of experimental computational sciences.

From a standpoint of computational methods, this section will clarify the procedure used for extracting values of interest from gate sweep curves, identifying regions of interest in Raman maps, map stitching, and $\mathrm{D} / \mathrm{G}$ ratio computation in a way which is scalable and limits arbitration bias. Analysis routines were written in 
the Python scripting language and can be found in A.

\section{Raman region identification}

In any form of data analysis, isolating a region of interest is of primary importance. In the case of the Raman maps presented in this study, the region of interest (the CNT channel) represents the minority of the acquired data. Indeed, the majority of the CNT-FETs analyzed compose less than $2.5 \%$ of the point spectra in a typical 40 $\mu \mathrm{m} \times 25 \mu \mathrm{m}$ map. It is therefore of great importance to clearly identify CNT spectra in the map and discard all others from further analysis.

To achieve this, a simple principal component analysis (PCA) algorithm was used. Spectra characteristic of the main regions $\left(\mathrm{SiO}_{2}\right.$ background, electrode edges, electrode bulk, and CNTs) were manually extracted from several maps and averaged to produce a spectrum dictionary. Cross-correlation coefficients were calculated and tracked for each point spectrum in the map, $I_{n}(\omega)$, and reference spectrum $I_{m}(\omega)$ according to the following relation:

$$
k_{n m}=\frac{1}{N} \sum_{\omega} \frac{\left(I_{n}(\omega)-\overline{I_{n}}\right)\left(I_{m}(\omega)-\overline{I_{m}}\right)}{\sigma_{n} \sigma_{m}}
$$

Where $N$ is the number of wavenumber values, $\omega$, or spectrum channels $(1,024)$ and $\sigma_{n}$ and $\sigma_{m}$ are the standard deviations of the map point and reference spectra, respectively: 


$$
\sigma=\sqrt{\frac{1}{N} \sum_{\omega}(I(\omega)-\bar{I})^{2}}
$$

Note the exceptional cases where $k_{n m}=-1, k_{n m}=0, k_{n m}=1$ indicate anticorrelation (e.g. $I_{n}(\omega)=-I_{m}(\omega)$ ), perfect uncorrelation (e.g. $I_{n}(\omega)$ or $I_{m}(\omega)$ is true noise), and perfect correlation $\left(I_{n}(\omega)=I_{m}(\omega)\right)$, respectively. Though this provides a strong method of spectrum categorization for several principal components, it was found that there was no functional improvement upon a $k_{n m}$ cut-off value for categorizing map points as CNTs. For this reason, the PCA algorithm was simplified slightly to only compute coefficicnets against the characteristic CNT spectrum, $k_{n, C N T}$, and employ a dynamic cutoff of $k_{\text {cut-off }}=\left(1-e^{-1}\right) \max \left(k_{n, C N T}\right)$.

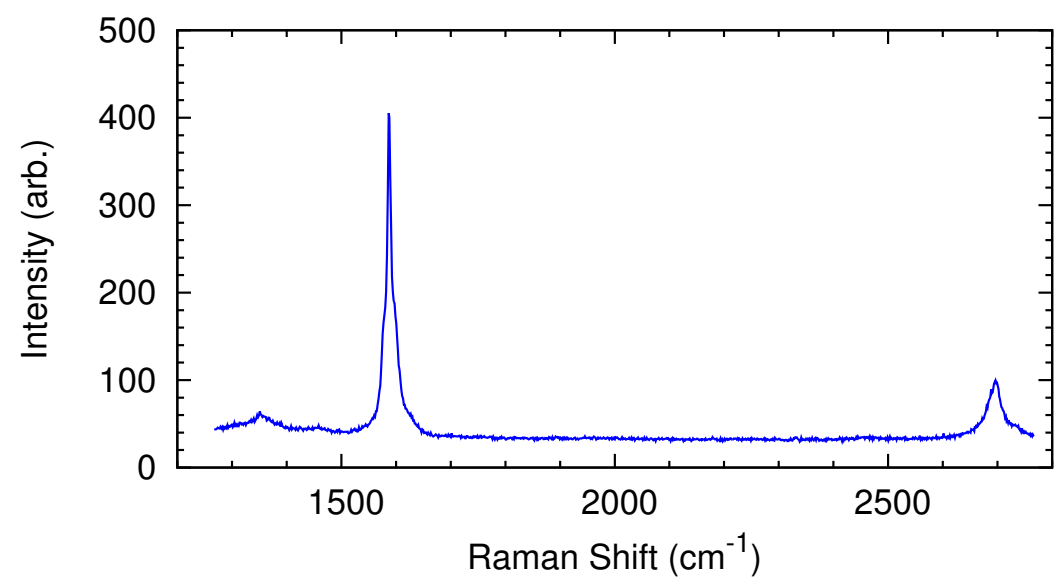

Figure 25: Averaged reference spectrum of a CNT used for PCA of all Raman maps

While the employed method utilizes an a priori reference spectrum, it is noted that a representative $n$-dimentional component space can be algrothmically achieved, albeit at greater computational expense. Briefly, this method is to generate a sum 
spectrum for the whole map, and then to locate regions in the map which have the lowest cross-correlation coefficient with the sum spectrum. In this way, the most linearly independent spectrum components are discovered. Given the fact that a single compound was under investigation with spectrum features sufficiently distinct from surrounding media, the spectrum dictionary method was sufficiently rigorous to isolate regions of interest. If, on the other hand, it was necessary to discriminate between CNTs and other $s p^{2}$ carbon compounds, a more algorithmic approach to the component discovery could be warranted.

\section{Raman map stitching}

Due to the arbitrary growth path of CNTs in the chemical vapor deposition (CVD) process, Raman mapping of CNT-FETs necessitates a degree of prospecting. The CNT channel can span the electrodes by the most direct path (across the $7 \mu \mathrm{m}$ gap between the electrode fingers) or by any number of meandering paths between the catalyst island and the electrode contact pad (some $50 \mu \mathrm{m}$ away). The acquisition time for Raman spectroscopy is an added complication. Even for modest acquisition parameters (2 second accumulations time, 3 averaged acquisition), a typical map for this study consisting of approximately 8,400 points would consume 14 hrs. Software overhead and stage translation can result in an additional 4 hours for such mapping

operations. This also presumes an ideal condition in which the sine bar governing the grating position does not requre adjustment. Without a sufficiently low groove 
density, actuating the sine bar to acquire the entire spectral region of interest could consume far more time than the data acquisition itself.

With these points in mind, the general procedure of mapping was to acquire an initial speculative map, then to collect any additional maps according to the expected CNT path. In the process of generating overall D/G ratio statistics, it was necessary to align and stitch together the successive maps and remove redundant points. Two behaviors of the commercial software and hardware resulted in added complications for this procedure: (1) Due to the small fixed range of the piezoelectric stage, it is only employed for translations in the mapping process. Shifting the stage, even within the bounds of the piezoelectric stage, employs the motorized stage. Due to gear backlash, the new mapping center is only accurate within a couple of microns. The absolute coordiates of the map origin must therefore be adjusted to compensate for gaps or excessive overlap depending upon the previous motorized stage translations. (2) In the process of Raman mapping, the recorded stage position corresponds with the true piezoelectric deflection rather than the user-specified mapping parameters. This results in a variation within about $10 \mathrm{~nm}$ of the user-specified values and thereby a variable mapping interval. This necessitates a special routine for managing the crosscorrelation of points in the maps which require a stitching operation. The entire map stitching operation can be outlined by the following procedure:

(1) For maps $M_{1}$ and $M_{2}$, collect all points within $M_{2}$ which lie in the potential overlap margin of $M_{1}$, defined as $M_{21}$. (2) Shift coordinates of all points in $M_{21}$ 
by $\overrightarrow{\delta_{n m}}=n d x \hat{\mathbf{i}}+m d y \hat{\mathbf{j}}$ where $n, m=\{-6 . .6\}$ and $d x, d y=350 \mathrm{~nm}$. That is, a $13 \times 13$ grid in increments of the user-specified mapping interval. (3) For all points in $M_{21}$ at a given $\overrightarrow{\delta_{n m}}$, compute the neareast point in $M_{1}$ (within a distance of $0.55 d x)$. (4) Compute cross-correlation coefficients between $M_{21}$ point spectra and their corresponding $M_{1}$ pair and determine the average coefficient, $k_{n m}$ for the given $\overrightarrow{\delta_{n m}}$. (5) Repeat for all values of $n$ and $m$ and shift $M_{2}$ by the $\overrightarrow{\delta_{n m}}$ value for which $k_{n m}$ is maximized. (6) Discard all points in the shifted $M_{2}$ which overlap $M_{1}$ and merge all points into a single map, $M_{f}$.

(a)

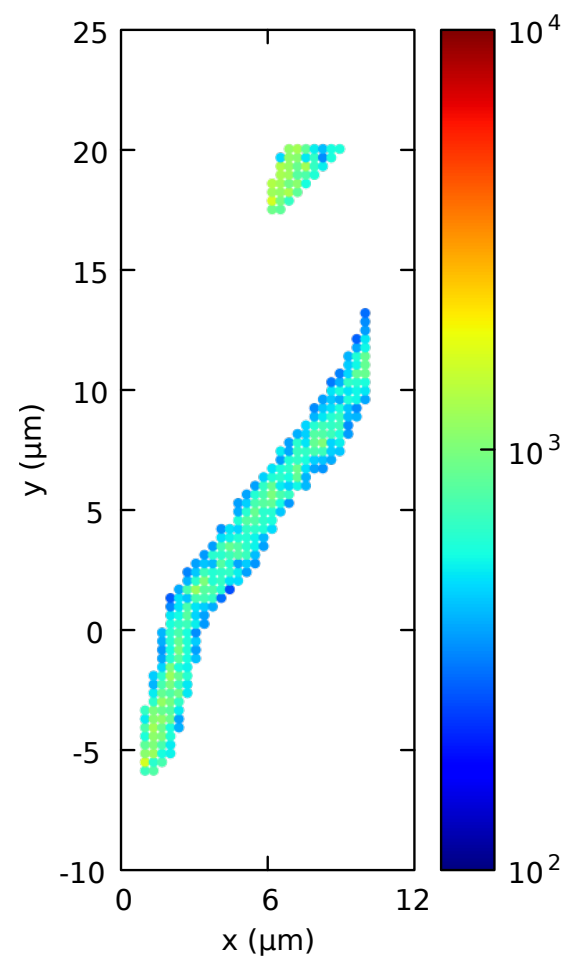

(b)

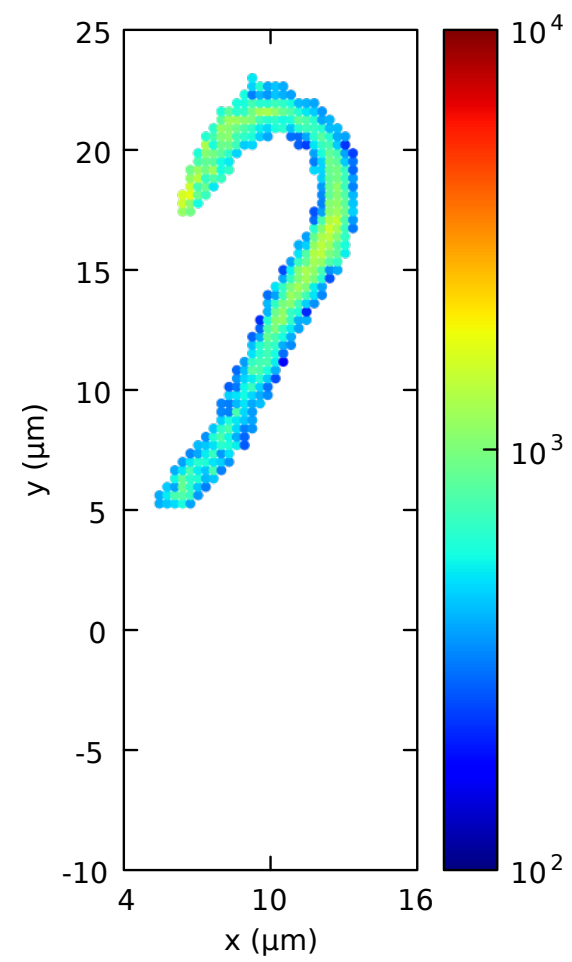

Figure 26: Overlapping Raman D-band intensity maps, (a) $M_{1}$ and (b) $M_{2}$ for a single device before any compositing function is applied.

From a standpoint of addressing the previously mentioned concerns, step 2 corrects for motor backlash whereas step 3 compensates for variation in piezoelectric 
stage read-out. The use of an averaged cross-correlation coefficient prevents biasing towards positions of maximum overlap. Figure 26 presents an example map of two overlapping Raman maps which require a stitching operation. The composite map is presented in Figure 27 before and after application of the stitching algorithm.

(a)

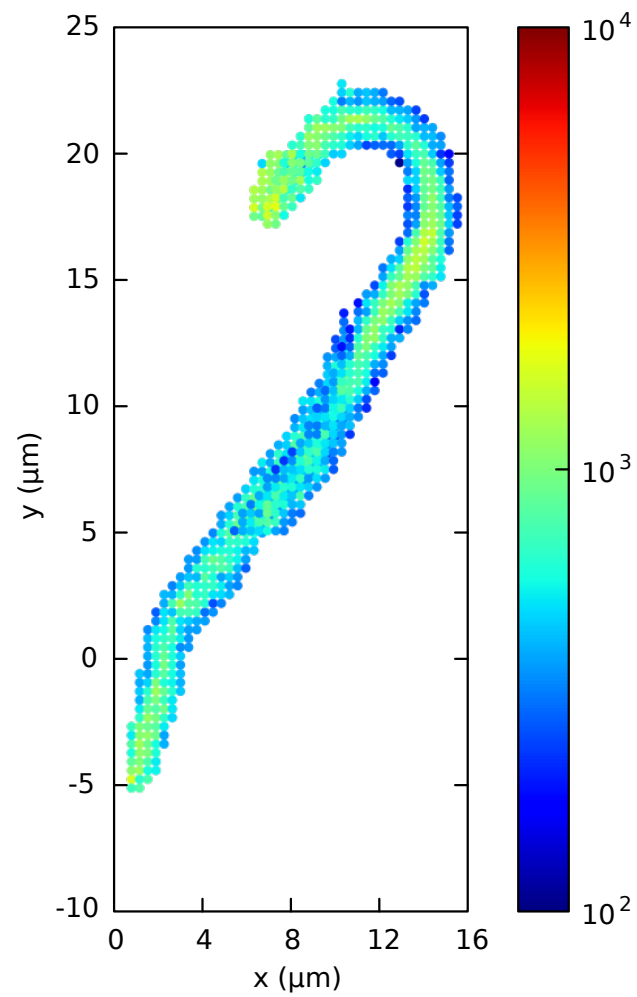

(b)

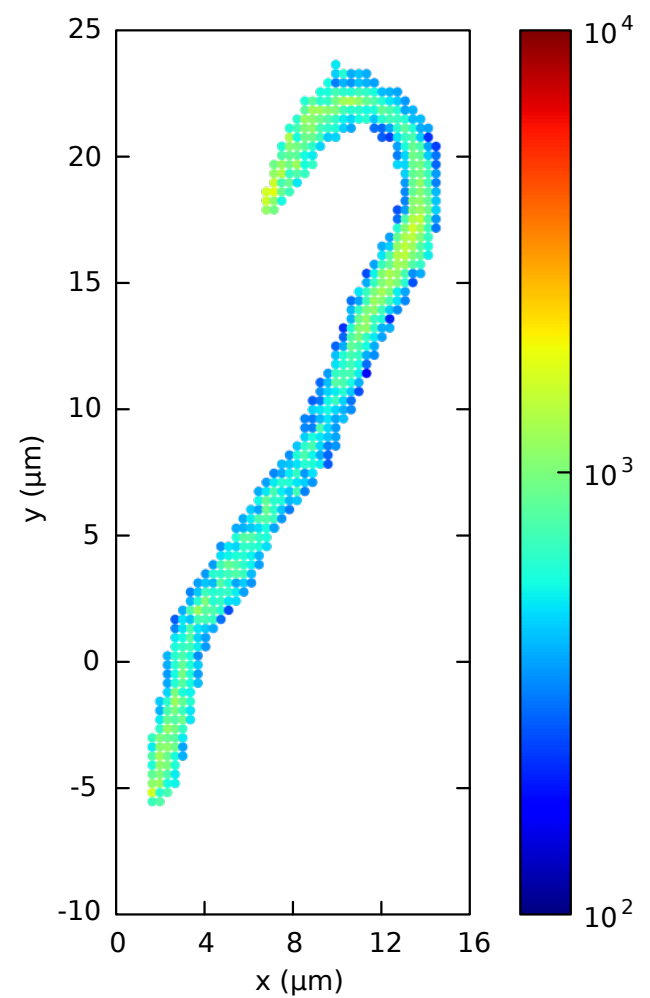

Figure 27: Composite Raman maps of D-band intensity merged by (a) direct overlay and (b) by the described map stitching algorithm.

For the example map presented, $M_{1}$ (Figure 26a) and $M_{2}$ (Figure 26b) consisted of 318 points and 400 points, respectively, while the overlap domain $M_{21}$ consisted of 131 points. The final stitched map, $M_{f}$ (Figure 27b) consisted of 566 points (i.e. 152 redundant points removed). Without applying the stitching algorithm, approximately $27 \%$ of the legitimate map points would be double-weighted in the final 
map statistics. Qualitative inspection of the stitched maps in Figure 26 shows that the redundant points were coinscident with a regional minimum in the D-band intensity. This highlights the importance of such a stitching procedure. Without applying the stitching algorithm (i.e. tolerating the overlap redundancy), the average $\mathrm{D} / \mathrm{G}$ ratio for the channel would be substantially reduced.

\section{Effective Schottky barrier}

As previously discussed in Section 2.4, the effective Schottky barrier, $\Phi_{S B}$, and threshold voltage, $V_{t h}$, will be used as metrics to discern any influence on the electronic transport properties of the CNT channel. As the thermionic emission regime greatly simplifies the relationship between measurable parameters and the Schottky barrier, computational methods must begin with locating this region of interest, followed by data/parameter extraction.

This was carried out by iterating a linear fit over a fixed gate voltage channel width (20 channels or $1 \mathrm{~V}$ ) along a gate-source curve, extracting the slope parameter from the fit, and isolating the range in which this slope (that is, the transconductance g) drops by no more than $10 \%$ of the maximum (where the thermionic transport mechanism is dominant). This is illustrated in Figure 28a.

The linear window of the thermionic emission regime, once located, is fit linearly (Figure 28b) to extract the threshold voltage, $V_{t h}$ (simply the $x$-intercept). To compute the change in effective Schottky barrier using only the information con- 
(a)

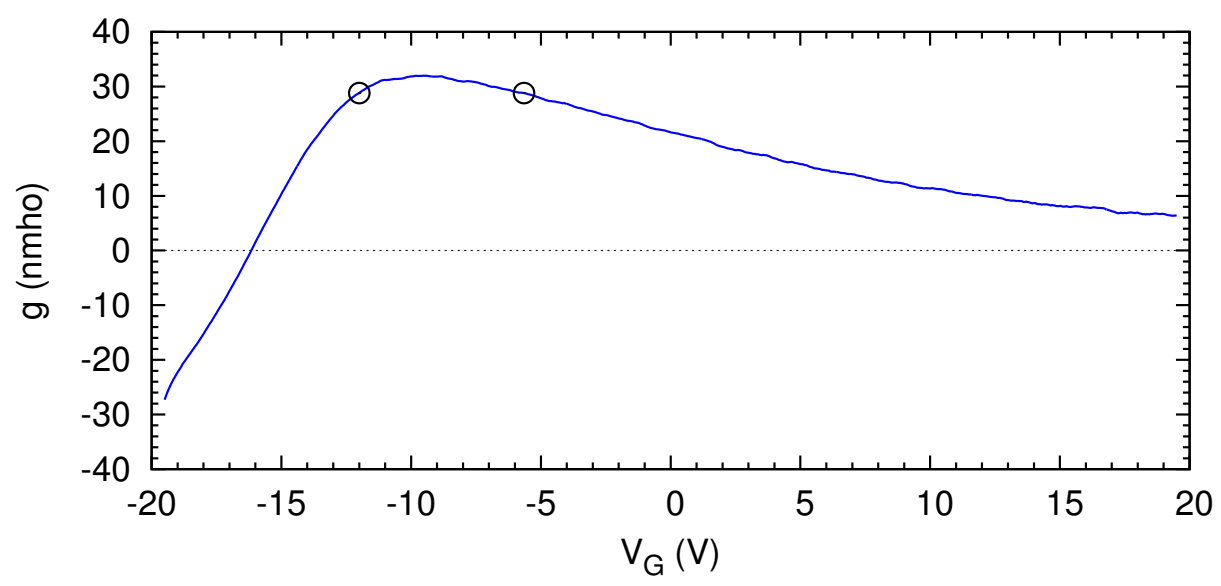

(b)

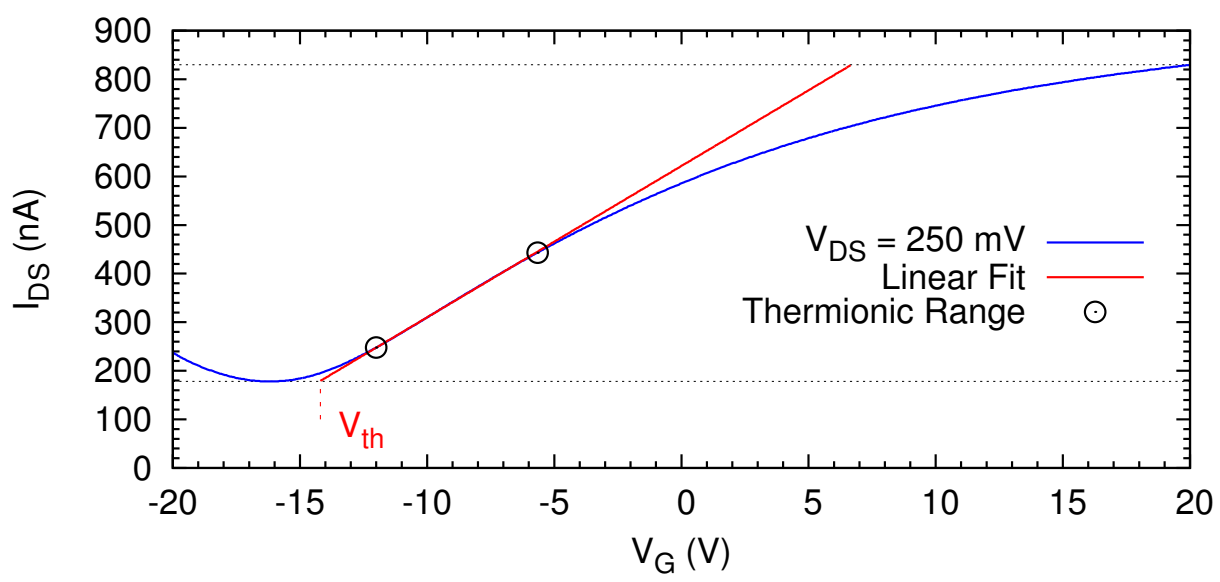

Figure 28: Location of thermionic emission regime in a single gate-source curve. (a) Transconductance, as determined by and iterated 20-channel linear fit with the thermioninc cut-off indicated and (b) linear fit of the defined regime in the full gate-source curve.

tained in the gate sweeps, $\Delta \Phi_{S B}$ must be computed for a static value of $V_{G}$ and $V_{D S}$. Therefore, the thermionic emission windows must be stored and compared to find overlapping regimes across chamber pressure settings. Once a range of overlap is determined, $\Delta \Phi_{S B}$ can be computed by re-arranging Equation 7 to solve for $\Phi_{S B}$ :

$$
\Phi_{S B}=-\frac{k_{B} T}{q}\left[\ln \frac{I_{S}}{A A_{G} T^{2}}\right]
$$


Where $A$ is the channel area (substituting $J_{S}=I_{S} / A$ ). The change in Schottky barrier height, $\Delta \Phi_{S B}$, from chamber pressure $P$ to $P^{\prime}$ can thereby be expressed as:

$$
\Delta \Phi_{S B}=\left[-\frac{k_{B} T}{q}\left(\ln I_{S}-\ln A A_{G} T^{2}\right)\right]_{P^{\prime}}-\left[-\frac{k_{B} T}{q}\left(\ln I_{S}-\ln A A_{G} T^{2}\right)\right]_{P}
$$

Noting that $A, A_{G}$, and $T$ do not change, the expression can be greatly simplified:

$$
\Delta \Phi_{S B}=\frac{k_{B} T}{q}\left[\ln \frac{I_{S}}{I_{S}^{\prime}}\right]
$$

Where $I_{S}$ and $I_{S}^{\prime}$ are the drain saturation current values at chamber pressures of $P$ and $P^{\prime}$, respectively, at a fixed $V_{G}$ value. This relationship can then be used to compute the change in effective Schottky barrier at a given value of $V_{G}$ which lies in the overlapping thermionic emission regimes of devices characterized under different chamber pressure conditions (the change in Schottky barrier due to the presence of adsorbates).

In order to compute the saturation current, $I_{S}$, Equation 6 can be re-written in the following form [60]:

$$
I_{D S}=I_{S} e^{\frac{q V_{D S}}{n k_{B} T}}\left(1-e^{-\frac{q V_{D S}}{k_{B} T}}\right)
$$

Instead of plotting $I_{D S}$ against $V_{D S}$, the natural logarithm of $I_{D S} /\left(1-\exp \left(-q V_{D S} / k_{B} T\right)\right)$ is plotted against $V_{D S}$ (Figure 29). Fitting the linear regime of this logarithmic plot 
(that is, the exponential region on a linear scale) allows the saturation current to be extracted from the $y$-intercept of the graph [60]. These values allow $\Delta \Phi_{S B}$ to be computed directly with using Equation 12.

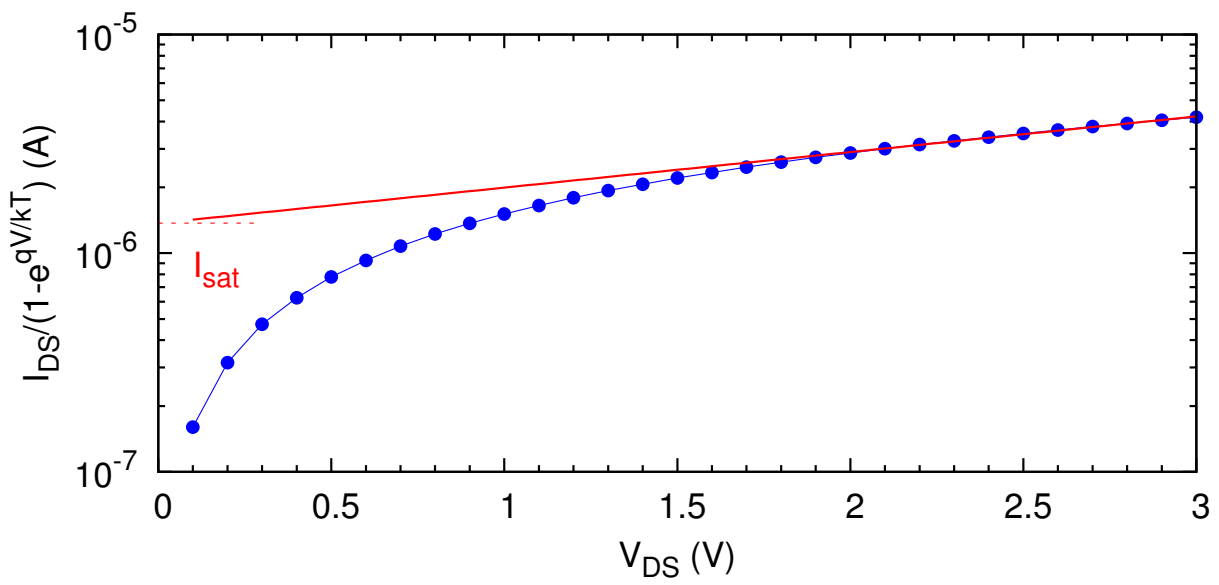

Figure 29: Logarithmic plot of drain-source current characteristics at a fixed gate voltage in the thermionic emission regime. Extrapolation of saturation current from the linear regime (on logarithmic scale) is illustrated. 


\section{Results}

\section{Device fabrication}

CNT-FETs were fabricated on conductively doped Si substrates with $100 \mathrm{~nm}$ of surface oxide. Catalyst islands $(1.0 \AA \mathrm{Fe})$ were patterned and deposited on the substrate using photolithography and magnetron sputtering. Growth of CNTs was carried out in a CVD reactor with a Joule-heated graphite stage. A base pressure of $8.5 \times 10^{-2}$ mbar was achieved before ramping the stage temperature to $925{ }^{\circ} \mathrm{C}$ at $800{ }^{\circ} \mathrm{C} / \mathrm{min}$. A carbon feedstock gas $\left(\mathrm{C}_{2} \mathrm{H}_{2}\right)$ and a reducing species $\left(\mathrm{H}_{2}\right)$ were allowed to flow into the chamber at 690 and $23 \mathrm{sccm}$, respectively (achieving a working pressure of $10 \mathrm{mbar}$ ). After 10 minutes, gas flow and heating current to the stance was ceased. Substrates were then allowed to cool to room temperature in vaccuum. Electrodes were then lithographically patterned and deposited by sputtering of $\mathrm{Au}(250 \mathrm{~nm})$. Devices were fabricated in large 10x10 grids of 6 devices (600 devices per die). Representative images of CNT-FETs are depicted in Figure 30. These fabrication procedures produced 66 operating devices (11\% yield). Devices which exhibited unfavorable electrical characteristics (diode, short circuit, or multi-channel) were excluded from this figure. Therefor, this figure does not represent the number of operational channels fabricated, but rather the pool of operational devices usable in this study. 
(a)

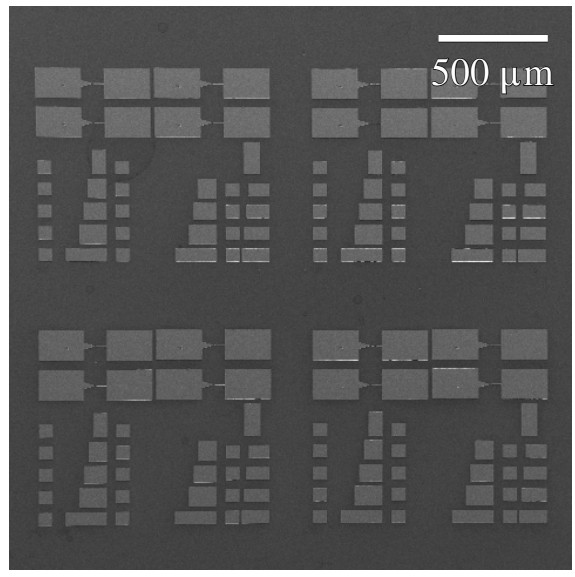

(b)

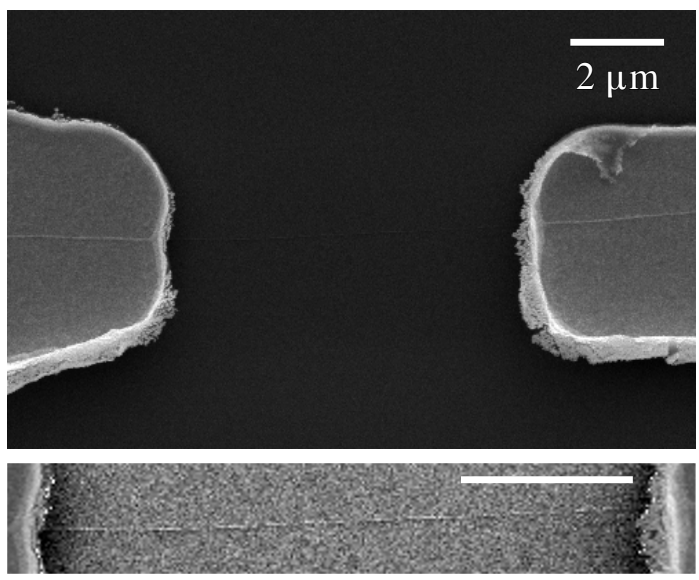

Figure 30: (a) Grid of sixteen CNT-FETs and accompanying test/alignment pads. (b) A single CNT-FET channel with contrast-enhanced channel showing bridging CNT (scale bar corresponds with the host image).

\section{Raman spectroscopy}

\subsubsection{Defect mapping}

Raman maps were collected from 31 single-channel CNT-FETs utilizing $4 \mathrm{~mW}$ of circularly polarized light through a 50x 0.55 NA objective (approximately $3.66 \times 10^{5}$ $\mathrm{W} / \mathrm{cm}^{2}$ ). While Raman map acquisition was previously illustrated in Figure 27b, Figure 31 shows representative post-computation D, G, and D/G ratio maps. Figure 31c shows that the defect density of this particular CNT exhibits a gradient in defect density. For the purposes of final analysis, these maps are reduced to a single statistic and therefore serve principally as a visual inspection mechanism to verify the output of computational work.

In order to prevent z-drift of the stage during mapping acquisition, the motorized stage was driven up towards the focal point, shifting the sample plane from 
(a)

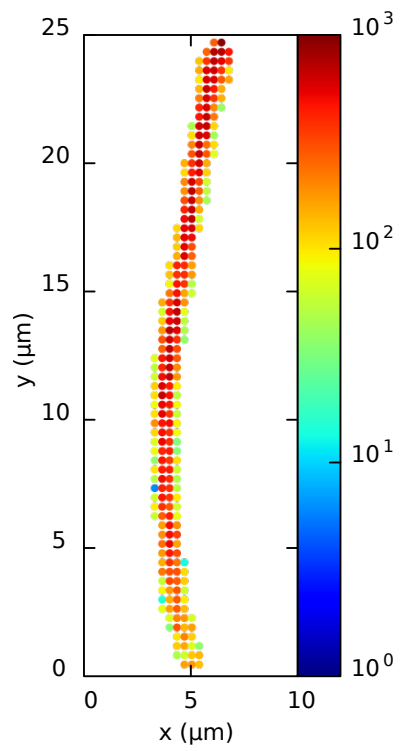

(b)

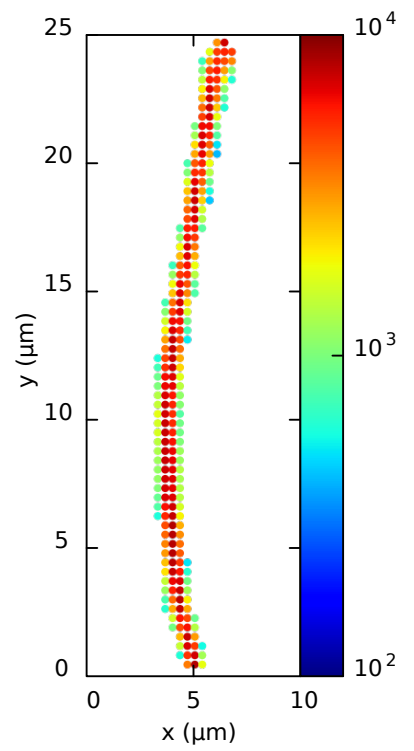

(c)

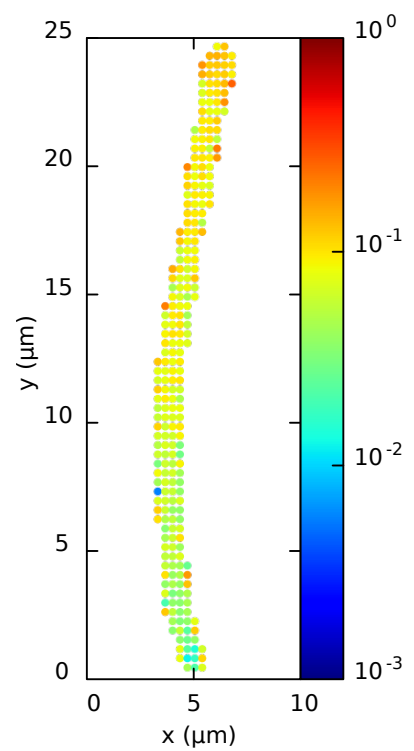

Figure 31: Raman spectrum maps of a CNT-FET collected with a circularly polarized source showing (a) D and (b) G peak integrated intensities. (c) Corresponding D/G ratio map.

an underfocused position. Allowing the stage to rest for two hours prior to mapping further assured that no settling of motor backlash would occur during the mapping acquisition process. No loss of spatial resolution was observed along the raster axis (parallel to the CNT channel), as shown in Figure 31, which suggests that any outof-plane stage drift was negligible.

\subsubsection{Defect quantification}

The defect maps (Figure 31c), as derived from the Raman D and G spectrum maps, are a useful means for visualizing the spatial distribution and overall variation in defect density. However, they do not present the defect density in a form which can facilitate comparison between CNTs. To satisfy this need, it is necessary to 
construct a defect histogram which shows the number of pixels in the map which fall into a specific interval of $D / G$ ratio. Examples of such histograms are presented in Figure 32, with the histogram featured in Figure 32a corresponding to the map presented in Figure 31. While there was some variation observed in defect density distribution and occasional mild asymmetry in distribution (such as in Figure 32b, no devices exhibited extraordinary (bimodal, for example) distributions.

(a)

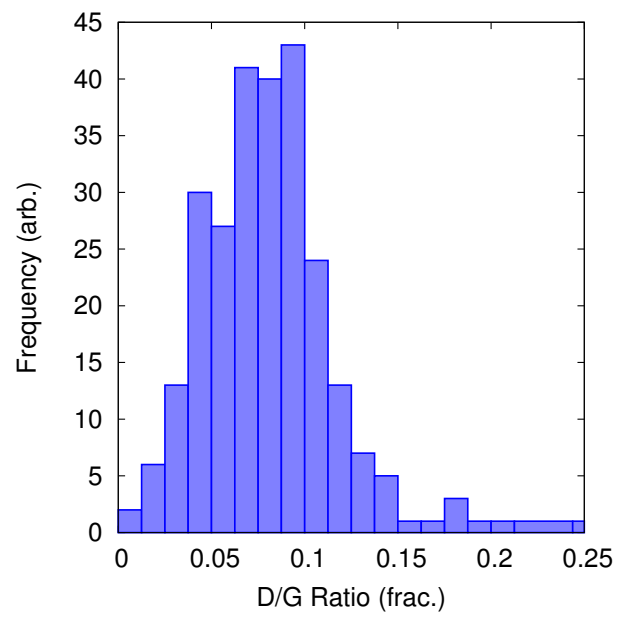

(b)

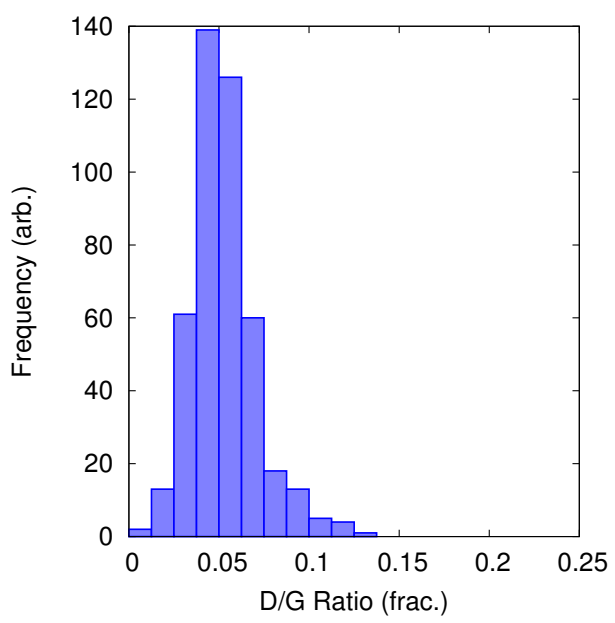

Figure 32: Histogram of $\mathrm{D} / \mathrm{G}$ ratio of an individual CNT channel with (a) broad distribution and (b) narrow distribution of defects.

Histograms of the $\mathrm{D} / \mathrm{G}$ ratios and number of Raman map points for the complete pool of devices included in the study can be seen in Figure 33, with 30-50 points being the most prevalent number points to be found in a map (corresponding with a direct growth between the tips of electrode fingers). Note that approximately $75 \%$ of CNTs have $\mathrm{D} / \mathrm{G}$ ratios less than 0.25 and that the population of measured devices has a relatively even distribution within this regime. 
(a)

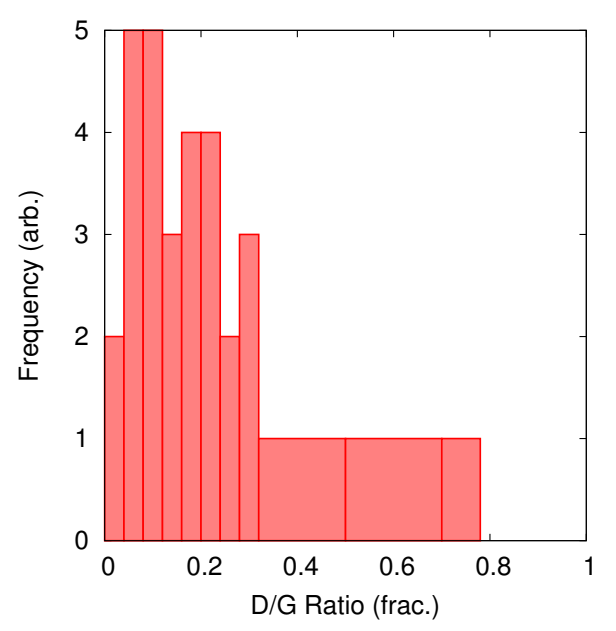

(b)

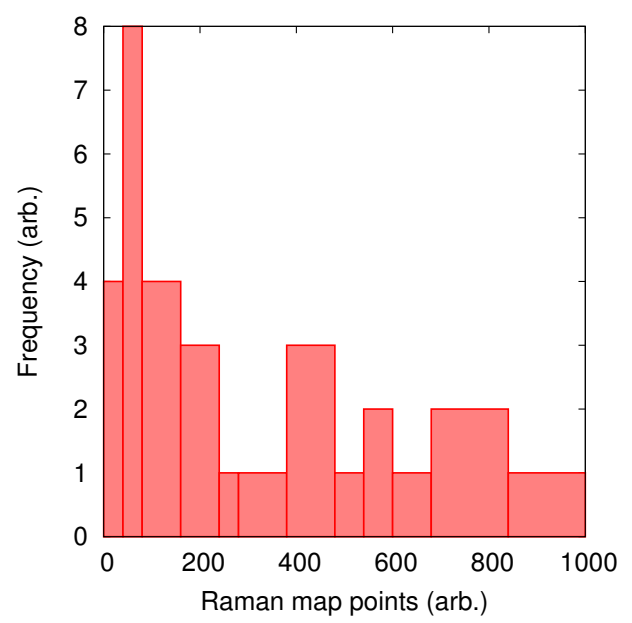

Figure 33: Histogram of (a) average D/G ratio and (b) number of Raman map points for all CNT-FETs included in this study.

\section{Electron transport}

Measurements of CNT-FET drain current were carried out in the MEPS at varied chamber pressures. In order to drive off adsorbates, the chamber and substrate were subjected to a $200{ }^{\circ} \mathrm{C}$ bake-out for 18 hrs after pumping down to to less than $5 \times$ $10^{-7}$ Torr. During the final 2 hrs of the bake-out cycle, a $15 \mathrm{~W} 250 \mathrm{~nm}$ UV lamp was used to illuminate the stage and chamber to assist with the desorption process. Measurements were carried out for each of 30 CNT-FET devices at three different chamber pressures: $3.5 \times 10^{-9}$ (base pressure), $3.0 \times 10^{-4}$, and $1.0 \times 10^{0}$ Torr. In order to elevate the chamber pressure, $\mathrm{O}_{2}$ gas was leaked into the chamber gradually using the leak valve. Prior to opening the leak valve, the leading gas lines were pumped and purged iteratively by rotary pump in order to reduce the amount of atmospheric air introduced into the chamber. Concentrations of various gases of 
interest were monitored using an RGA (Figure 24 depicts conditioning of the chamber from $3.5 \times 10^{-9}$ to $5 \times 10^{-6}$ Torr). The chamber atmosphere was found to be $>95 \%$ $\mathrm{O}_{2}$ under such conditions.

(a)

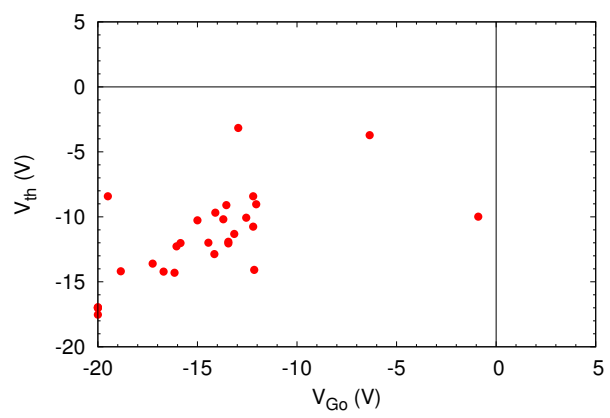

(c)

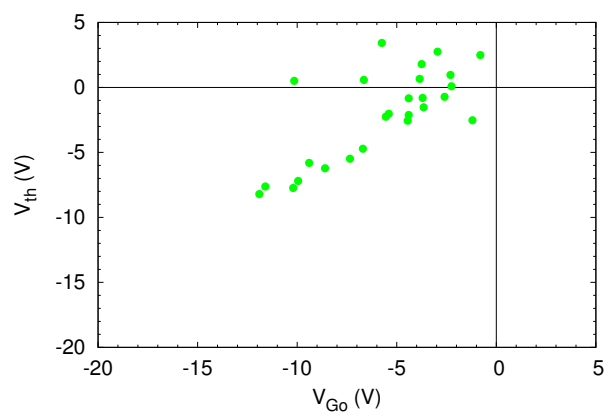

(b)

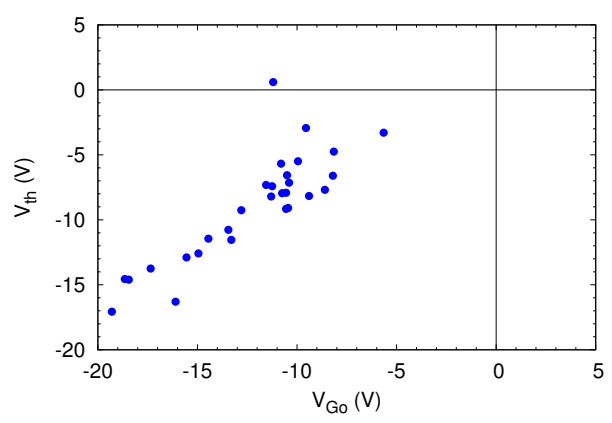

(d)

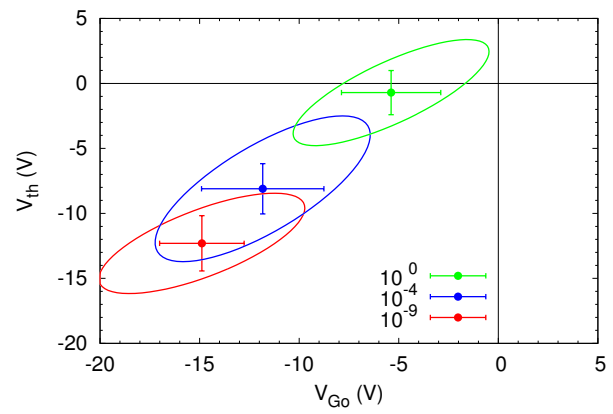

Figure 34: Comparison of threshold and off-state gate voltages for each CNT-FET at (a) $3.5 \times 10^{-9}$, (b) $3.0 \times 10^{-4}$, and (c) $1.0 \times 10^{0}$ Torr chamber pressure $\left(V_{D S}=250 \mathrm{mV}\right)$. (d) Centroid of (a-c). Error bars indicate one standard deviation centered about the average $V_{G o}$ and $V_{t h}$ values.

At each previously mentioned pressure setting, gate-source sweeps were collected. Gate voltages were varied from -20 to $20 \mathrm{~V}$ in $50 \mathrm{mV}$ increments with $V_{D S}$ ranging from $-1 \mathrm{~V}$ to $1 \mathrm{~V}$ in $250 \mathrm{mV}$ increments. Gate voltage off-state $\left(V_{G o}\right)$ and threshold voltage values $\left(V_{t h}\right)$ were computed from the gate sweeps in accordance with Section 5.3. They are presented in correlative form for each pressure setting in Figure 34. Figure 34d conjointly shows the centroids of all three data clusters to 
illustrate the progression in $V_{G o}$ and $V_{t h}$ with respect to pressure. These data are similarly expressed in Figure 35, showing that $V_{t h}$ tends to remain approximately 3.5 $\mathrm{V}$ higher than $V_{G o}$ at each pressure setting.

(a)

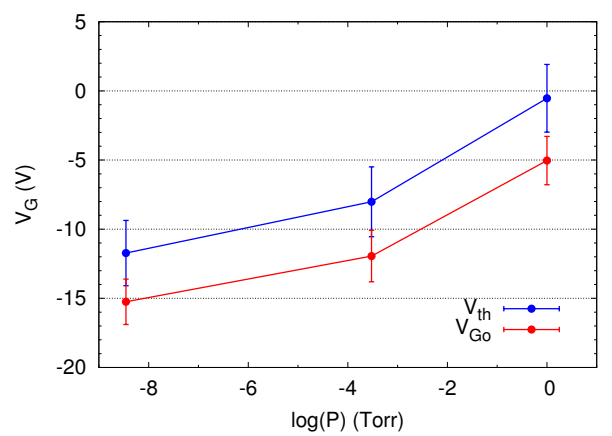

(b)

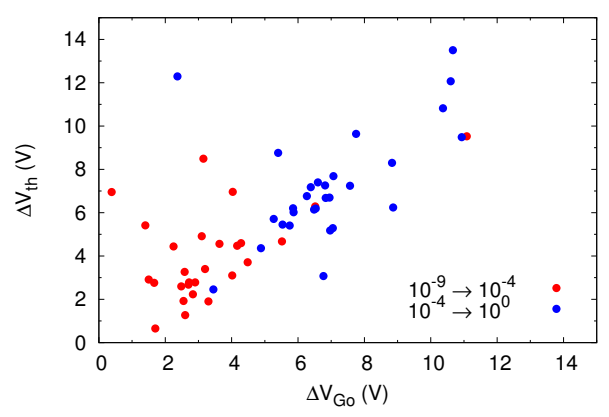

Figure 35: (a) Average change in $V_{G o}$ and $V_{G k}$ with respect to chamber pressure and (b) corresponding changes for the two chamber pressure transitions $\left(3.5 \times 10^{-9}\right.$ to $3.0 \times 10^{-4}$ Torr and $3.0 \times 10^{-4}$ to $1.0 \times 10^{0}$ Torr) .

\section{Correlations}

Having presented the general statistics individually observed in Raman spectroscopy and electronic characterization of the CNT-FETs in the MEPS, the two must be correlated. Figure 36 shows correlative plots of the change in off-state and threshold gate voltage $\left(\Delta V_{G o}\right.$ and $\left.\Delta V_{t h}\right)$ with respect to computed $\mathrm{D} / \mathrm{G}$ ratios and number of Raman map points for each device across the two pressure transitions.

No clear correlation is observed between the gate voltage shifts and the number of map points (which translates directly to the length of the CNT), or with the defect density as determined by the $\mathrm{D} / \mathrm{G}$ ratio. It is however noted that the shorter channels (less than approximately 100 map points) exhibit a larger spread of gate voltage 
(a)

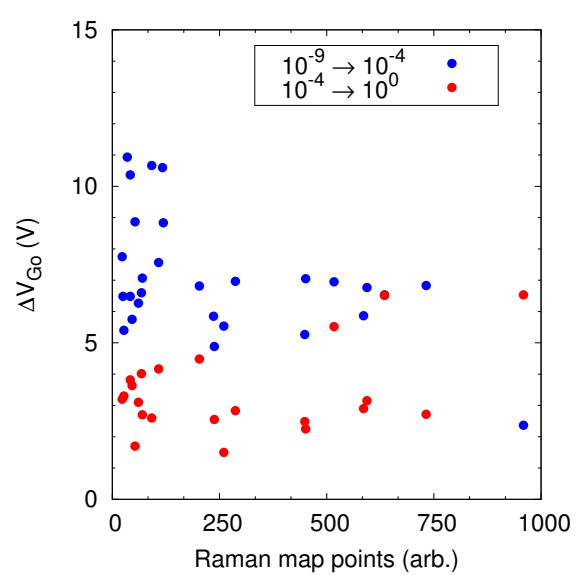

(c)

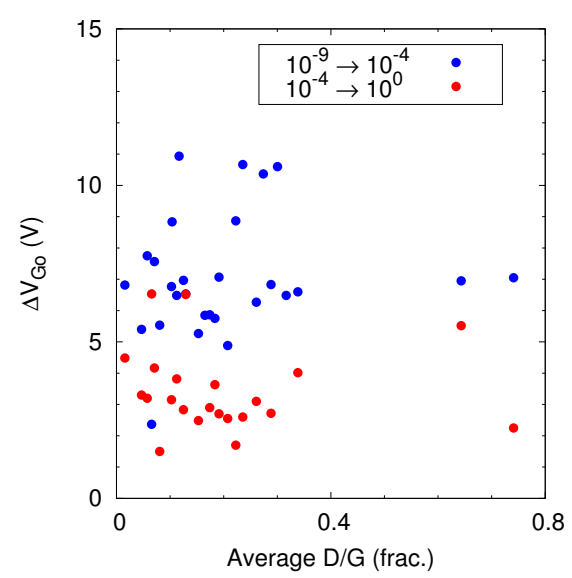

(b)

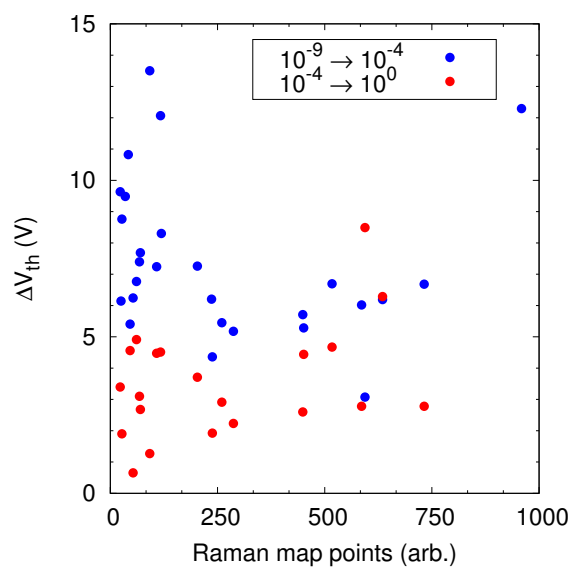

(d)

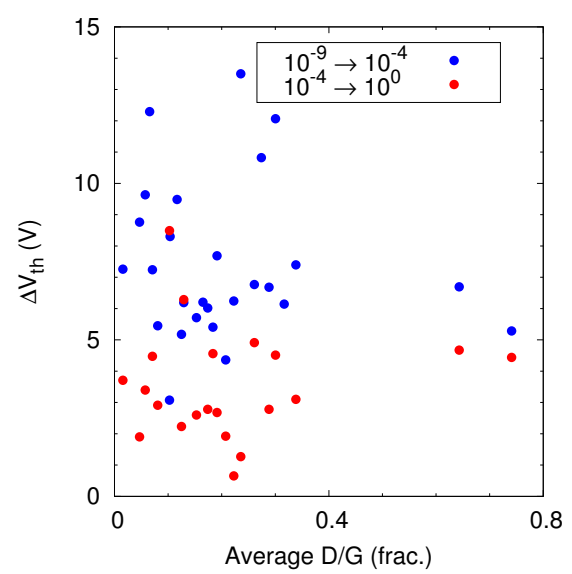

Figure 36: Correlative data showing relationship between (a) gate voltage off-state shift and (b) threshold voltage shift as a function of Raman map points for both chamber pressure transitions $\left(3.5 \times 10^{-9}\right.$ to $3.0 \times 10^{-4}$ Torr and $3.0 \times 10^{-4}$ to $1.0 \times 10^{0}$ Torr $)$. (c) Similar data showing the relationship between gate voltage off-state shift and (d) threshold voltage shift as a function of Raman D/G ratio.

shifts, as shown in Figure 36a and Figure 36b. Though this observation is of limited statistical significance due to the small population of devices with greater channel lengths.

When the change in effective Schottky barrier, $\Delta \Phi_{S B}$ is computed (in accordance with the methods presented in Section 5.3) and correlated to these two Raman-derived quantities, the lack of correlation is reinforced. A broad spread is 
(a)

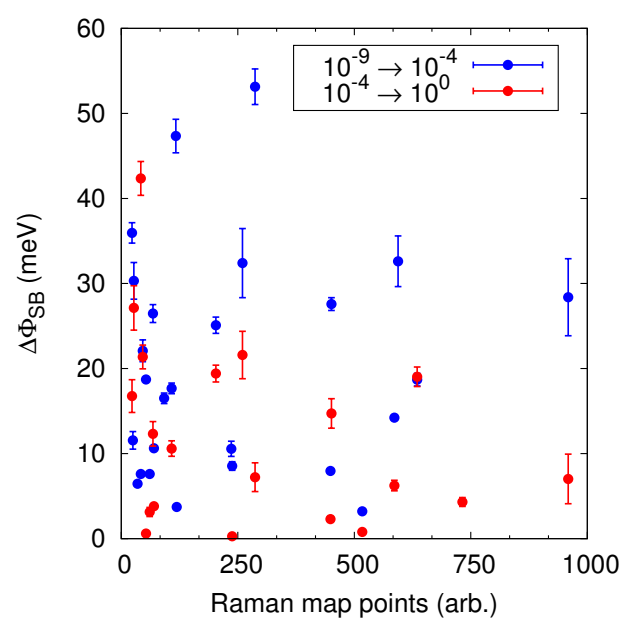

(b)

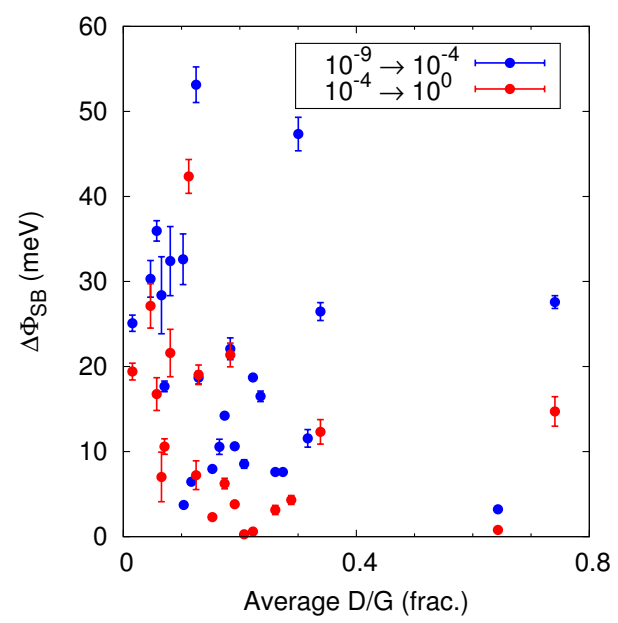

Figure 37: Correlative data showing relationship between change in effective Schottky barrier and (a) number of Raman map points and (b) Raman D/G ratio for both chamber pressure transitions $\left(3.5 \times 10^{-9}\right.$ to $3.0 \times 10^{-4}$ Torr and $3.0 \times 10^{-4}$ to $1.0 \times 10^{0}$ Torr $)$. Error bars represent one standard deviation.

observed in the change in barrier height, independent of the defect density or channel length. Though it can be noted that the error bars about $\Delta \Phi_{S B}$ are relatively small which indicates that the barrier height varies very little within the thermionic emission regime (with average deviations of approximately $16 \%$ and $5 \%$ for the first and second pressure transitions, respectively). 


\section{Discussion}

As mentioned in Section 6.4, Figure 36 and Figure 37 clearly show a lack of correlation between any of the device characteristics (shifts in $V_{t h}$ and $V_{G o}$, or changes in $\Phi_{S B}$ ) and the $\mathrm{D} / \mathrm{G}$ ratio. These data suggest that the defect density of the CNT channel does not play a substantial role in the device performance and gas sensing metrics. Specifically, the defects do not play a dominant role in the adsorption process and the impact of adsorbates on the device characteristics. In addition, the number of map points (indirectly, the length of the CNT channel) similarly plays no role. Even when considered jointly via a defect-to-length ratio, no correlation is observed. It is therefore necessary to assess, in more concrete terms, what role defects are capable of playing in the modulation of the CNT transport properties in the presence of oxygen.

This will be addressed by considering the following: (1) Quantification of the atomic defect density in the CNT channel and comparison to dopant concentrations; (2) Gas-surface collisions and associated statistics; (3) Chemisorption, physisorption, and charge transfer processes. These concepts, reconciled with the presented data, will be used to propose a model for the gas sensitivity observed.

\section{Defect density estimation}

In the work by Cançado, an empirical formula was determined for computing the absolute defect density of graphene as a function of the Raman D/G ratio $[43,50$, 61]. Expressed in two forms, this formula showed that the average distance between 
defects, $L_{D}(\mathrm{~nm})$, and defect density, $n_{D}\left(\mathrm{~cm}^{-2}\right)$, were given by:

$$
\begin{aligned}
& L_{D}^{2}=\left(1.8 \pm 0.5 \times 10^{-9}\right) \lambda^{4}\left(\frac{I_{D}}{I_{G}}\right)^{-1} \\
& n_{D}=\left(1.8 \pm 0.5 \times 10^{22}\right) \lambda^{-4}\left(\frac{I_{D}}{I_{G}}\right)
\end{aligned}
$$

Where $I_{D}$ and $I_{G}$ are the intensities of the Raman D and G peaks, and $\lambda$ is the Raman excitation wavelength $(\mathrm{nm})$. Note that this relationship does not hold for relatively high defect densities $\left(L_{D} \leq 10 \mathrm{~nm}\right)$ where a decrease in $I_{D} / I_{G}$ is observed, though severe broadening of the $\mathrm{D}, \mathrm{G}$, and $2 \mathrm{D}$ peaks allow this regime to be easily identified. Assuming that these relations hold for CNTs, much like electronic band structure and portions of the phonon dispersion relation (and thereby, Raman phenomena generic to graphitic compounds), the Raman map data can be used to estimate absolute defect densities of the CNT-FETs in the study.

Using the average D/G ratios for each CNT channel, the average distance between defects was found to be $34.2 \mathrm{~nm}\left(\sigma=17.4 \mathrm{~nm}, n_{D}=4.64 \times 10^{10} \mathrm{~cm}^{-2}\right)$. By combining these values with Raman region map data, an absolute defect quantification can be performed.

The CNT channel surface area can be estimated by first converting the number of Raman map points into an approximate length. As observed in Figure 31, the CNT channel has a diameter of 3-4 points (which was typical among the maps), which 
coincides with the Raman excitation spot diameter of 1.18 umand typical spatial resolution of Raman maps. The CNT channel length can be estimated by dividing the number of map points by 3.5 and multiplying by the mapping interval $(350 \mathrm{~nm})$. In estimating the surface area of the CNT, absent a chirality measurement, an assumption might also be made concerning the diameter. While such an assumption may seem to be impose a cost to the precision in defect quantification, the functional quantity in this case is the atomic surface density of the CNT, which is invariant of diameter.

Having arrived at an estimated CNT length and surface area $(A=\pi d L)$, the raw number of atomic defect sites, $N_{D}$ can be trivially computed. Similarly, knowing the approximate linear atomic density of a $2 \mathrm{~nm}$ diameter CNT (58.37 atoms/nm) facilitates computation of the number of carbon atom sites, $N_{C}$, in the CNT channel. This allows the fraction of defect sites, $N_{D} / N_{C}$, to be considered in the same way one would the dopant concentration in a bulk semiconductor. While dopant concentrations are often expressed in terms of volume, the atomic ratio provides a more direct analog. On average, the defect fraction was found to be $4.99 \times 10^{-5}$ (with a minimum of $3.77 \times 10^{-6}$ and a maximum of $\left.1.79 \times 10^{-4}\right)$.

To compare this with commercial bulk semiconductors, silicon has an atomic concentration of approximately $5 \times 10^{22}$ atoms $/ \mathrm{cm}^{3}$ and dopant concentrations can vary between $1 \times 10^{13}$ and $1 \times 10^{18}$ atoms $/ \mathrm{cm}^{3}$, depending upon the various desired transport properties. That is, in atomic terms, the dopant fraction is between $1 \times 10^{-9}$ 
and $1 \times 10^{-4}$, which encompasses the range of defect densities present in the CNTs in this study. It is important to note that this parallel is only important in so far as these surface defects are considered adsorption sites. It is not expected that intrinsic defects in such concentrations would result in substantial change to electrical characteristics.

\section{Gas-surface collisions}

If the gas sensing properties of the CNT-FETs is to be attributed to interactions between surface defects and gas molecules, the nature of gas-surface interactions must be discussed as well as the nature of the surface defects. To compute the surface collision rate, it is first necessary to determine the average velocity of a gas molecule and the molecular flux upon a surface in a chamber, which are given by $[62,63]$ :

$$
\begin{aligned}
& \bar{v}=\sqrt{\frac{8 k_{B} T}{\pi m}} \\
& \phi=\bar{v} N_{v} A / 4
\end{aligned}
$$

Where $m$ is the mass of the molecule, $N_{v}$ is the number of molecules per unit volume, and $A$ is the area of the surface of interest. The flux of $\mathrm{O}_{2}$ molecules incident upon the CNT channel can thereby be estimated in terms of pressure as follows:

$$
\phi=\frac{P}{\sqrt{2 \pi k_{B} T m_{O_{2}}}}
$$


Using values of $295 \mathrm{~K}$ and $31.9998 \mathrm{amu}$ for the temperature and molecular mass of diatomic oxygen, respectively, the flux was determined to be $94.9,8.14 \times 10^{6}$, and $2.71 \times 10^{10} \mathrm{um}^{-2} \mathrm{~s}^{-1}$ for chamber pressures of $3.5 \times 10^{-9}, 3.0 \times 10^{-4}$, and 1.0 Torr, respectively (and $\bar{v}_{O_{2}}=441.8 \mathrm{~m} / \mathrm{s}$ ). From $\phi$, it is trivial to compute the number of collisions per second, $z=\phi A$, of the CNT channel (where $A$ is the area of the CNT as estimated in Section 7.1). Correspondingly, with the previously computed defect densities, The average collision rate with the aggregate lattice defects in the entire channel can also be computed. These values are summarized in Table 1.

\begin{tabular}{c|l|r|r|r}
\cline { 3 - 5 } \multicolumn{2}{c}{} & \multicolumn{3}{c}{$P$ (Torr) } \\
\cline { 3 - 5 } \multicolumn{2}{c}{$\mathrm{CNT}$} & $3.5 \times 10^{-9}$ & $3.0 \times 10^{-4}$ & $1.0 \times 10^{0}$ \\
\hline \multirow{3}{*}{ Defect } & $z_{\max }\left(\mathrm{s}^{-1}\right)$ & $5.72 \times 10^{1}$ & $4.90 \times 10^{6}$ & $1.63 \times 10^{10}$ \\
& $\bar{z}\left(\mathrm{~s}^{-1}\right)$ & $1.72 \times 10^{1}$ & $1.47 \times 10^{6}$ & $4.90 \times 10^{9}$ \\
& $z_{\min }\left(\mathrm{s}^{-1}\right)$ & $1.37 \times 10^{0}$ & $1.18 \times 10^{5}$ & $3.92 \times 10^{8}$ \\
\hline \multirow{2}{*}{$z_{\max }\left(\mathrm{s}^{-1}\right)$} & $4.82 \times 10^{-3}$ & $4.13 \times 10^{2}$ & $1.38 \times 10^{6}$ \\
& $\bar{z}\left(\mathrm{~s}^{-1}\right)$ & $9.46 \times 10^{-4}$ & $8.11 \times 10^{1}$ & $2.70 \times 10^{5}$ \\
& $z_{\min }\left(\mathrm{s}^{-1}\right)$ & $1.82 \times 10^{-5}$ & $1.56 \times 10^{0}$ & $5.20 \times 10^{3}$ \\
\hline
\end{tabular}

Table 1: Collision rates of $\mathrm{O}_{2}$ molecules at various pressures with entire CNT channel and with defect sites.

\section{Chemisorption and physisorption processes}

Having established statistics concerning the number of lattice defects in the CNT channel and collisions between this surface and ambient $\mathrm{O}_{2}$ molecules, it is necessary to discuss the actual interaction between gas molecules and the CNT surface. This will provide the conceptual linkage between the gas-surface collisions and the pressure dependence of the threshold voltage. 
The most extreme adsorption case is that of chemisorption, a process involving the formation of new chemical bonds between the CNT channel and adsorbed gas species. An intuitive consideration suggests that such higher binding energy interactions (on the order of several electron volts $[62,63]$ ) are unlikely in this scenario. Indeed, given that kinetic collisions are the sole mechanism available to initialize the binding event, a room temperature gas is not expected to be capable of causing an ionization event. Integrating the Maxwellian energy distribution for $\mathrm{O}_{2}$ suggests a probability of $6.86 \times 10^{-51}$ that an $\mathrm{O}_{2}$ molecule will have kinetic energy greater than or equal to $3 \mathrm{eV}$ under these conditions. First principles computations have yielded binding energies between 3.58 and $5.06 \mathrm{eV}$ for chemisorbed $\mathrm{O}_{2}$ onto CNTs of various chiralities [64], suggesting that even this probability is a conservative estimate. With the aforementioned literature values, probabilities of sufficient kinetic energy would range between $9.25 \times 10^{-61}$ and $5.71 \times 10^{-86}$. With an estimated $10^{19}$ gas molecules in the chamber at 1.0 Torr, the probability that a single molecule has sufficient kinetic energy to initialize chemisorption is vanishingly small.

In further studies, it has been shown that surface-adsorbed $\mathrm{O}_{2}$ exhibits minimal change in characteristic stretch frequencies relative to the gas phase (from 194 to 191 $\mathrm{eV}$ as measured by electron energy-loss spectroscopy) $[65,66]$. This is in contrast with the expected energy of $140 \mathrm{eV}$ for chemisorbed $\mathrm{O}_{2}^{-}$species [67]. This further supports the notion that chemisorption of $\mathrm{O}_{2}$ presents a negligible fraction of the gas-surface interactions. 
By contrast, physisorbed molecules are bound by Van der Waals with binding energies on the order of a fraction of an electron volt. For graphitic carbon, reported binding energies associated with $\mathrm{O}_{2}$ physisorption ranged from 104 to 120 meV $[68,69]$. Physisorption onto single CNTs occurs with a slightly higher binding energy, with reported values ranging from 120 to $192 \mathrm{meV}$, depending upon chirality and orientation of the adsorbed molecule $[54,56,69]$. In these same studies, it was computed that a charge transfer of approximately $0.1 q_{e}$ occurs at the adsorbate site (electron donation by the CNT) $[54,56,59]$, suggesting that adsorbed $\mathrm{O}_{2}$ molecules can act as p-type dopants. Repeating the same integral of the Maxwellian distribution yields a probability between $2.40 \times 10^{-2}$ and $1.73 \times 10^{-3}$ that a given $\mathrm{O}_{2}$ molecule will have kinetic energy greater than 120 to $192 \mathrm{meV}$.

\section{Dynamic nanotube-adsorbate model}

In Section 7.1 it was demonstrated that the CNT defect densities are on the same order of magnitude as dopant densities in traditional commercial semiconductors. This lends casual plausibility to the notion that they play an important role as adsorbate acceptor sites and possibly contributed to the hypothesis that chemisorbed molecules at these defect sites is the cause of threshold voltage shifts in CNT-FETs, and further, that the quantity of defects determines the effective doping level of the CNT channel. The correlations presented in Section 6.4 clearly show that the lattice defects play no such determining role in determining the effective doping level. 
The subsequent revelations in Section 7.2 and Section 7.3, in which collision rates and adsorption binding energies were discussed, further suggest that the gas sensitivity of CNT-FETs may not be attributable to a static dopant concentration model. If the CNT-FET were to be examined solely as a comparison between UHV and atmospheric states, a static adsorption model may seem plausible. However, given the collision rates for the intermediate pressure range $\left(1.47 \times 10^{6} \mathrm{~s}^{-1}\right.$ at $3.0 \times 10^{-4}$ Torr), it is clear that an adsorbed monolayer will form so as to make the intermediate indistinguishable from the low vacuum regime (1.0 Torr). Periodic measurements of the gate-source curves at various pressures show that threshold ranges are stable for weeks at a time. This observation is strictly incompatible with a static dopant model (though not necessarily independent of defect-enhanced adsorption).

A much more plausible mechanism is a dynamic adsorption-desorption model. Some fraction of excited electrons created by biasing the CNT-FET scatter off of the adsorbed $\mathrm{O}_{2}$ molecules, desorbing them from the CNT surface. The incident flux of $\mathrm{O}_{2}$ molecules results in a pressure-dependent radsorption rate. The balancing of these adsorption and desorption rates results in a steady-state dopant concentration which is characteristic of the operating pressure. That is, the change in dopant concentration (per unit area), $n_{d}$ is governed by an expression of the following form:

$$
N_{T} \frac{d n_{d}}{d t}=\nu N_{O_{2}} e^{-E_{d} / E_{q}}-s \phi N_{L}
$$

Where $N_{T}$ is the total number of adsorption candidate sites in the channel, $N_{L}$ is 
the number of such lattice sites available for adsorbates, $N_{O_{2}}$ are the number of sites occupied by adsorbates, $s$ is the sticking coefficient (a temperature-dependent adsorption probability), $E_{d}$ is the desorption activation energy (approximately equal to the adsorbate binding energy in the case of physisorption), $E_{q}$ is the energy of the charge carriers, $\phi$ is the molecular flux as defined by Equation 18, and $\nu$ is a pre-exponential frequency factor.

The first term containing the exponential is a modified version of the spontaneous thermal desorption equation $[62,63]$. In the thermal case, $\nu$ is a temperaturedependent coefficient which represents the vibrational frequency of surface adsorbed molecules, given by $\nu=k_{B} T / h$ (effectively the number of opportunities per second a molecule is likely experience a desorption event, approximately $6.25 \times 10^{12} \mathrm{~s}^{-1}$ at room temperature). In the case of current-induced desorption, $\nu$ must be dependent upon $I_{D S}$ instead, as the electrons are responsible for the desorption events. Another substitution made is $E_{q}$ in place of the thermal energy quantity, $k_{B} T$. Again, as the desorption events are caused by electron-adsorbate scattering rather than thermal excitations, the energy supply to overcome $E_{d}$, is dependent upon the energy of the charge carriers and therefore must depend, in some manner, upon $V_{D S}$, the FET bias. An important accompanying note is that the electronic transitions in the CNT, as viewed in the Katura plot (Figure 6b), are on the same order of magnitude of the physisorbed $\mathrm{O}_{2}$ binding energies, indicating that there a wealth of potential relaxation states for the desorption-initiating electrons. This also provides a context for potential 
chirality-dependent effects which would explain a lack of correlation between the threshold voltage shifts and both the defect density, and the CNT channel length. In a purely static adsorption model for the dopant concentration, a correlation with either would be observed.

It is also of great importance that this proposed model does not rule out the possibility that lattice defects play some role in adsorption of ambient gas molecules. Indeed, from a theoretical standpoint, it has been shown that defect sites facilitate adsorption [20]. However, the data from this correlative study (Figure 37) suggest that the defects are not the primary indicator of the threshold voltage shift (and thereby the dopant concentration).

If a future study were to find that the defects are the principal adsorption sites, the proposed model (Equation 19) could be modified such that $N_{T}$ would represent the number of defects. In such a case, the terms $\nu$ and $E_{q}$, which are dependent upon device operation (and thereby CNT chirality), must play a dominant role in determining the desorption/adsorption equilibrium condition. Absent this fact, a correlation between threshold voltage shift and $\mathrm{D} / \mathrm{G}$ ratio would have been observed in Section 6.4. In this way, the defect density could determine the maximum dopant density (which, as mentioned, is on a similar order of magnitude to bulk semiconductor dopant densities) while the gas molecule collisions and the electronic structure of the CNT determine the steady-state dopant concentration. 


\section{Charge traps and transport}

Another concept of interest in the field of defects and dopants is deep-level trap states. Deep-level traps are spatially localized mid-gap electronic states, usually in the vicinity of defects or impurities. Excited charge carriers fall into these trap states and ultimately recombine with their corresponding opposing carrier non-radiatively by releasing a phonon (known as the Shockley-Read-Hall process) [60]. One indicator of deep-level trap states is a broadening of the transition between the "on" and "off" states observed in a gate-source curve (that is, a reduction in transconductance). As charge carriers are injected into the channel, a larger gate voltage must be applied to fill the gap states in order to reach saturation. In addition, the reduced occupancy caused by the presence of the trap states results in a downward shift of the Fermi level and can thereby the Schottky barrier, causing a shift in the threshold voltage.

This effect can be observed qualitatively in Figure 17d due to increased oxygen concentration (a slight decrease in the slope of the curve for $V_{G}>V_{G o}$ as the chamber pressure is increased). As the other computational methods already required computation of the transconductance in the thermionic emission regime (Section 5.3), extrapolation of $\Delta g$ (with respect to pressure) for statistical analysis was a straightforward endeavor. These data showed that the average transconductances were 66.1, 59.0, and $37.1 \mathrm{nmhos}$ for chamber pressures of $3.5 \times 10^{-9}, 3.0 \times 10^{-4}$, and 1.0 Torr, respectively. Average percent changes in transconductance for each pressure transition were observed to be $-10.7 \%$ and $-37.1 \%$ (from lowest to highest pressure, with 
$83.3 \%$ of these changes in transconductance being negative). The correlation of the threshold broadening with the chamber pressure clearly indicates that the surface adsorbed oxygen are acting as charge traps, decreasing the transconductance.

Further, no correlation was observed between either the transconductance or the change in the transconductance and the defect density (at static chamber pressures), which indicates that the defects do not intrinsically provide deep trap states and that it's unlikely that a trapping effect at the defect sites is being enhanced by the adsorbed oxygen. Rather, the deep-level trap correlation with pressure could support the dynamic adsorption-desorption model, wherein the desorption of $\mathrm{O}_{2}$ molecules is the energy transfer mechanism by which charge carriers recombine (non-radiatively). Photodesorption studies of CNTs have also noted that there is a strong case for a desorption mechanism induced by excited charge carrier production and recombination [70]. 


\section{Conclusion}

This study has shown that there is no observable statistical correlation between either threshold voltage shift, or change in effective Schottky barrier at CNT-electrode junctions, due to the presence of oxygen adsorbates and the lattice defect density of the CNT channel. This suggests that adsorption of ambient gas molecules onto CNT surface defects is not the principal mechanism for oxygen sensitivity of CNT-FETs.

Further, evaluating the change in transconductance with respect to chamber

pressure provided evidence that oxygen molecules generate deep trap states in the CNT channel which are responsible for the corresponding threshold voltage shift. A dynamic adsorption-desorption model was proposed which allows for adsorption sensitivity, the potential for defect-enhanced adsorption effects, and the lack of correlation observed. A corresponding functional form for this model was also described. This model, which includes a charge carrier-induced desorption mechanism, suggests that there could be chirality-dependent effects which drive the variation in threshold voltage shifts observed between devices. In the process of this discovery, a variety of instrumentation and analytical developments have been made.

With respect to Raman spectroscopy, various software and hardware developments have allowed more complex polarization-dependent measurements to be made. Large scale map analysis was carried out using a custom Python library which can be adapted and applied to many other spectrum mapping applications. In addition, the hardware control of a half-wave plate provides opportunities for even greater develop- 
ments in Raman mapping with the following adaptations: (1) Polarization mapping functionality: Incident polarization could be used as an additional parameter, extending the spectrum map into a data cube which provides the option of either mapping polarization-dependent effects or, alternatively, to integrate the data cube, providing a polarization-independent map. (2) Polarization-feedback mapping: By inserting an an analysis function during acquisition of the polarization-dependent data cube, the polarization could be dynamically adjusted and a mapping routine could be crafted to follow polarization-dependent contours in the Raman map. This would increase spectrum intensity for polarization-sensitive materials such as CNTs by maintaining optimal polarization orientation and, in the process, reduce map acquisition time by following the projected polarization path.

The development of the MEPS was also a substantial achievement of this study. The MEPS presents a robust and adaptable platform for applying a large number of atmospheric stimuli to specimen while collecting a similar variety of measurements. While most measurements presented in this study which utilize the MEPS reproduce existing work (absent correlative Raman data), Section 8.2 outlines some existing and potential capabilities which may be used to measure previously unobserved phenomena. 


\section{Broader impact}

In the field of gas sensors, the results of this study will have substantial impact for CNT- and graphene-based devices. Under the previously conceived mechanisms for the impact of oxygen on CNT-FET transport, control over the defect density of the channel would be critical. The results of this study indicate that tuning the defect density within reasonable limits will not improve or degrade detection limits of CNTbased (and likely graphene-based) sensors. The defect density of CNTs and graphene can be tuned to some degree during the growth by varying the ratio between the carbon feedstock and reducing species gases, or alternatively, by post-processing via ion bombardment. Eliminating the defect density as a parameter of interest can guide researchers and engineers in their endeavor to optimize these carbon-based sensors.

Development and modeling of next generation CNT-based electron field emission sources may also benefit from the conclusions of this study. Due to their high aspect ratio and ease of vertical alignment, CNTs show promise as electron filaments. One major concern in extending emitter lifetime is surface contamination. In this sense, developing a better understanding of the mechanisms for gas adsorption will contribute greatly towards developing models for, and protocols to extend, emitter lifetime. In addition, characterizing the mechanisms behind ambient gas sensitivity will inform emitter design and fabrication. For instance, by tuning the chirality of the CNT emitter so as to reduce any variation in emission threshold due to trace gas contaminants. 
With respect to the field of CNTs and graphene in general, the defect quantification methods presented provide an opportunity for advancing measurement protocols. Introducing the $\mathrm{D} / \mathrm{G}$ ratio histogram as a new standard for defect density characterization may be advantageous. The vast majority of studies which concern CNT defect densities in some fashion rely solely on a single spectrum to characterize the entire channel. It is immediately apparent, upon inspecting the $\mathrm{D} / \mathrm{G}$ ratio histograms (Figure 32), that this is a wholly inadequate extrapolation. A comprehensive assessment of defect density must include some notion as to amount of variation throughout the specimen, which is provided directly in the $\mathrm{D} / \mathrm{G}$ ratio histogram.

\section{Further studies}

While the present study has offered a number of new insights to the field of CNTFET gas sensors, there are additional avenues to broaden impact of this study and provide a deeper understanding of any CNT-adsorbate phenomena. Beyond simply increasing the statistical population of devices, three possible expansions are offered.

\subsubsection{Differentiation: metallic vs. semiconducting}

As previously mentioned in Section 2.1, a subset of chiralities have energy bands at the Fermi level, resulting in a hybrid of conductor and semiconductor characteristics. Some computational efforts by Kang et al. $[53,54]$ suggest that the adsorption of $\mathrm{O}_{2}$ molecules to the surface of a CNT results in the creation of additional states near the 
Fermi level. The presence of pre-existing mid-gap states in the metallic CNTs may result in a different trend than those which are semiconducting. Specifically, smaller changes in the Schottky barrier shift, or perhaps other more dominant secondary effects (less change in transconductance due to the charge traps).

\subsubsection{Photostimulated transport}

In recent years, photonic stimulation and photo-emission spectroscopy of CNT-FETs have been of interest to researchers [71-74]. By measuring the gate-source characteristics while irradiating the CNT channel with a tunable IR source, it has been shown that the chirality of the CNT can be determined by correlating a peak in photocurrent with the wavelength of the excitation source. However, these studies have only been carried out under atmospheric conditions. In addition, none have considered the effects of the photonic stimulation on the back-gate substrate, conductively doped silicon, which could increase the local charge density and thereby mimic a change in gate voltage. In the process of this study, the MEPS system was fitted with a fiber optic feed-through and tunable IR source Figure 38, making it the ideal tool to address these issues.

(a)

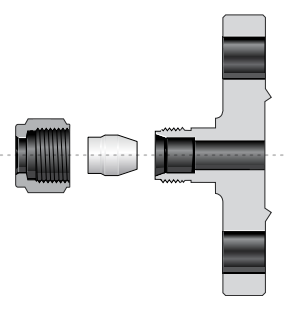

(b)

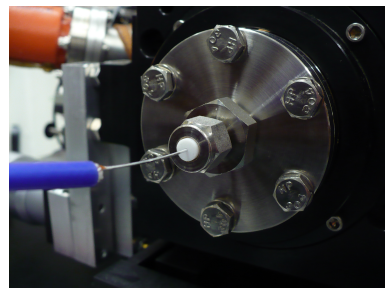

(c)

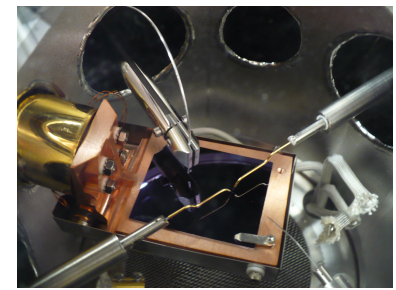

Figure 38: (a) Cross-section of custom feed-through with Teflon ferrule. Photographs of (a) optical fiber feed-through and (b) clamping fiber armature above a specimen. 


\subsubsection{Kelvin probe measurements}

With the work function of electrode materials being such a critical factor in device performance (being responsible in part for determining the Schottky barrier height), it stands to reason that a simultaneous in situ work function measurement would be beneficial. In this way, effects due to fluctuations in the source and drain electrodes could be subtracted and result in a pure measurement of the CNT channel. This could be achieved by allocating a probe port with a Kelvin probe (Figure 39). The accompanying schematic (Figure 39b) shows how an AC signal, $V(t)$ can be applied in a decoupled fashion to drive the the probe with a height adjustment potential, $U_{1}$. The induced current, fed through a lock-in amplifier and integrator, can be read out on an ammeter. The compensating potential, $U_{2}$, can then be adjusted to reduce the measured current to zero. At which point, $U_{2}$ will be equal to the difference in work function between the probe and the measured specimen.

(a)

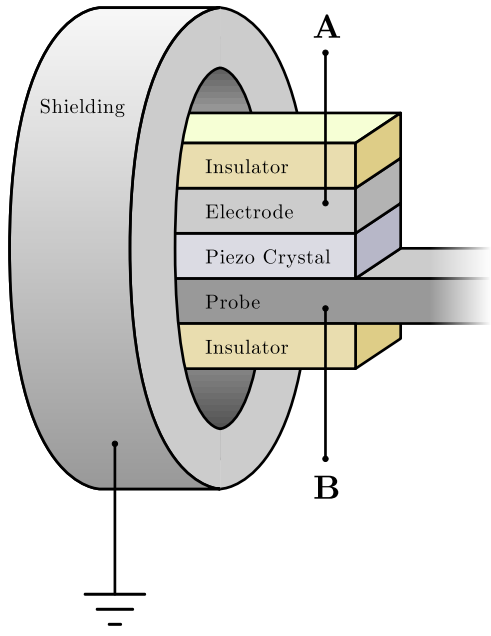

(b)

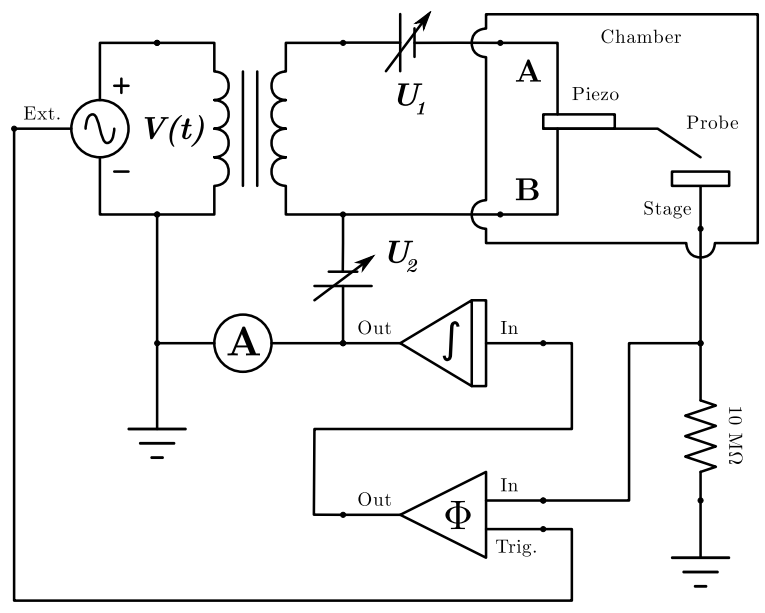

Figure 39: Schematic of (a) Kelvin probe feed-through and (b) control and measurement electronics. 


\section{References}

[1] S. Iijima, Nature, 1991, 354, 56-58.

[2] S. Saito, Science, 1997, 278, 77-78.

[3] A. Naeemi, J. D. Meindl, Annual Review of Materials Research, 2009, 39, 255-275.

[4] J. Y. Huang, S. Chen, Z. Q. Wang, K. Kempa, Y. M. Wang, S. H. Jo, G. Chen, M. S. Dresselhaus, Z. F. Ren, Nature, 2006, 439, 281-281.

[5] A. Thess, R. Lee, P. Nikolaev, H. Dai, P. Petit, J. Robert, C. Xu, Y. H. Lee, S. G. Kim, A. G. Rinzler, D. T. Colbert, G. E. Scuseria, D. Tománek, J. E. Fischer, R. E. Smalley, Science, 1996, 273, 483-487.

[6] K. Chu, Q. Wu, C. Jia, X. Liang, J. Nie, W. Tian, G. Gai, H. Guo, Composites Science and Technology, 2010, 70, 298 - 304.

[7] A. K.-T. Lau, D. Hui, Composites Part B: Engineering, 2002, 33, 263 - 277.

[8] J. Wang, Electroanalysis, 2005, 17, 7-14.

[9] J.-M. Bonard, J.-P. Salvetat, T. Stöckli, W. A. d. Heer, L. Forró, A. Châtelain, Applied Physics Letters, 1998, 73, 918-920.

[10] W. Zhu, C. Bower, O. Zhou, G. Kochanski, S. Jin, Applied Physics Letters, 1999, $75,873-875$.

[11] S. Fan, M. G. Chapline, N. R. Franklin, T. W. Tombler, A. M. Cassell, H. Dai, Science, 1999, 283, 512-514.

[12] G. Pirio, P. Legagneux, D. Pribat, K. B. K. Teo, M. Chhowalla, G. A. J. Amaratunga, W. I. Milne, Nanotechnology, 2002, 13, 1.

[13] J. I. Sohn, S. Lee, Y.-H. Song, S.-Y. Choi, K.-I. Cho, K.-S. Nam, Applied Physics Letters, 2001, 78, 901-903.

[14] D. H. Lee, J. E. Kim, T. H. Han, J. W. Hwang, S. Jeon, S.-Y. Choi, S. H. Hong, W. J. Lee, R. S. Ruoff, S. O. Kim, Advanced Materials, 2010, 22, 1247-1252.

[15] J. Li, Q. Ye, A. Cassell, H. T. Ng, R. Stevens, J. Han, M. Meyyappan, Applied Physics Letters, 2003, 82, 2491-2493.

[16] S. Heinze, J. Tersoff, R. Martel, V. Derycke, J. Appenzeller, P. Avouris, Physical Review Letters, 2002, 89.

[17] A. Javey, J. Guo, Q. Wang, M. Lundstrom, H. Dai, supernovae, 1999, 118, 1766-1776. 
[18] P. G. Collins, Science, 2000, 287, 1801-1804.

[19] D. McClain, N. Thomas, S. Youkey, R. Schaller, J. Jiao, K. P. O'Brien, Carbon, 2009, 47, 1493-1500.

[20] N. Govind, J. Andzelm, A. Maiti, IEEE Sensors Journal, 2008, 8, 837-841.

[21] M. Dresselhaus, G. Dresselhaus, R. Saito, A. Jorio, Physics Reports, 2005, 409, 47-99.

[22] M. S. Dresselhaus, A. Jorio, M. Hofmann, G. Dresselhaus, R. Saito, Nano Letters, 2010, 10, 751-758.

[23] V. N. Popov, P. Lambin, Physica Status Solidi B, 2009, 246, 2602-2605.

[24] G. S. Duesberg, I. Loa, M. Burghard, K. Syassen, S. Roth, Physical review letters, 2000, 85, 5436-5439.

[25] A. M. Rao, A. Jorio, M. A. Pimenta, M. S. S. Dantas, R. Saito, G. Dresselhaus, M. S. Dresselhaus, Physical review letters, 2000, 84, 1820-1823.

[26] M. S. Dresselhaus, A. Jorio, A. G. Souza Filho, R. Saito, Philosophical Transactions of the Royal Society of London A: Mathematical, Physical and Engineering Sciences, 2010, 368, 5355-5377.

[27] K. S. Novoselov, A. K. Geim, S. V. Morozov, D. Jiang, M. I. Katsnelson, I. V. Grigorieva, S. V. Dubonos, A. A. Firsov, Nature, 2005, 438, 197-200.

[28] K. S. Novoselov, D. Jiang, F. Schedin, T. J. Booth, V. V. Khotkevich, S. V. Morozov, A. K. Geim, Proceedings of the National Academy of Sciences of the United States of America, 2005, 102, 10451-10453.

[29] A. H. Castro Neto, F. Guinea, N. M. R. Peres, K. S. Novoselov, A. K. Geim, Rev. Mod. Phys., 2009, 81, 109-162.

[30] J.-H. Chen, W. G. Cullen, C. Jang, M. S. Fuhrer, E. D. Williams, Physical review letters, 2009, 102, 236805.

[31] E. H. Martins Ferreira, M. V. O. Moutinho, F. Stavale, M. M. Lucchese, R. B. Capaz, C. A. Achete, A. Jorio, Phys. Rev. B, 2010, 82, 125429.

[32] D. Graf, F. Molitor, K. Ensslin, C. Stampfer, A. Jungen, C. Hierold, L. Wirtz, Nano Letters, 2007, 7, 238-242.

[33] R. Saito, M. Fujita, G. Dresselhaus, M. S. Dresselhaus, Applied Physics Letters, 1992, 60, 2204-2206. 
[34] H. Kataura, Y. Kumazawa, Y. Maniwa, I. Umezu, S. Suzuki, Y. Ohtsuka, Y. Achiba, Synthetic Metals, 1999, 103, 2555-2558.

[35] R. Saito, G. Dresselhaus, M. S. Dresselhaus, Physical Review B, 2000, 61, 2981.

[36] J. Maultzsch, S. Reich, C. Thomsen, H. Requardt, P. Ordejón, Phys. Rev. Lett., 2004, 92, 075501.

[37] J. Maultzsch, S. Reich, C. Thomsen, Physical Review B, 2004, 70, 155403.

[38] A. C. Ferrari, J. C. Meyer, V. Scardaci, C. Casiraghi, M. Lazzeri, F. Mauri, S. Piscanec, D. Jiang, K. S. Novoselov, S. Roth, A. K. Geim, Physical Review Letters, 2006, 97.

[39] F. Tuinstra, The Journal of Chemical Physics, 1970, 53, 1126.

[40] I. Pócsik, M. Hundhausen, M. Koós, L. Ley, Journal of Non-Crystalline Solids, 1998, 227-230, Part 2, 1083 - 1086.

[41] C. Thomsen, S. Reich, Phys. Rev. Lett., 2000, 85, 5214-5217.

[42] A. Ferrari, Solid State Communications, 2007, 143, 47-57.

[43] K. Sato, R. Saito, Y. Oyama, J. Jiang, L. Cancado, M. Pimenta, A. Jorio, G. Samsonidze, G. Dresselhaus, M. Dresselhaus, Chemical Physics Letters, 2006, 427, 117-121.

[44] R. Saito, T. Takeya, T. Kimura, G. Dresselhaus, M. S. Dresselhaus, Physical Review B, 1998, 57, 4145.

[45] A. Jorio, M. A. Pimenta, A. G. Souza Filho, R. Saito, G. Dresselhaus, M. S. Dresselhaus, New Journal of Physics, 2003, 5, 139.

[46] W. Shockley, Electrons and Holes in Semiconductors: With Applications to Transistor Electronics, of The Bell Telephone Laboratories series, D. Van Nostrand, 1959.

[47] E. Rhoderick, R. Williams, Metal semiconductor contacts, 2nd. Edn., Oxford university press, 1988.

[48] C. Casiraghi, A. Hartschuh, H. Qian, S. Piscanec, C. Georgi, A. Fasoli, K. S. Novoselov, D. M. Basko, A. C. Ferrari, Nano Letters, 2009, 9, 1433-1441.

[49] M. Lucchese, F. Stavale, E. M. Ferreira, C. Vilani, M. Moutinho, R. B. Capaz, C. Achete, A. Jorio, Carbon, 2010, 48, 1592-1597. 
[50] L. G. Cancado, A. Jorio, E. H. M. Ferreira, F. Stavale, C. A. Achete, R. B. Capaz, M. V. O. Moutinho, A. Lombardo, T. S. Kulmala, A. C. Ferrari, Nano Letters, 2011, 11, 3190-3196.

[51] A. Jorio, R. Saito, J. H. Hafner, C. M. Lieber, M. Hunter, T. McClure, G. Dresselhaus, M. S. Dresselhaus, Physical Review Letters, 2001, 86, 1118-1121.

[52] K. Besocke, S. Berger, Review of Scientific Instruments, 1976, 47, 840-842.

[53] D. Kang, N. Park, J. Hyun, E. Bae, J. Ko, J. Kim, W. Park, Applied Physics Letters, 2005, 86, 093105.

[54] D. Kang, N. Park, J. Ko, E. Bae, W. Park, Nanotechnology, 2005, 16, 1048.

[55] K. Bradley, J.-C. P. Gabriel, M. Briman, A. Star, G. Gruner, Physical Review Letters, 2003, 91.

[56] S. Dag, O. Gülseren, T. Yildirim, S. Ciraci, Phys. Rev. B, 2003, 67, 165424.

[57] A. Tchernatinsky, S. Desai, G. U. Sumanasekera, C. S. Jayanthi, S. Y. Wu, B. Nagabhirava, B. Alphenaar, Journal of Applied Physics, 2006, 99, 034306.

[58] M. Hatami, A. Farmany, R. Sahraei, Fullerenes, Nanotubes and Carbon Nanostructures, 2014, 22, 434-453.

[59] H. Chang, J. D. Lee, S. M. Lee, Y. H. Lee, Applied Physics Letters, 2001, 79, 3863-3865.

[60] D. K. Schroder, Semiconductor material and device characterization, John Wiley \& Sons, 2006.

[61] L. G. Cancado, K. Takai, T. Enoki, M. Endo, Y. A. Kim, H. Mizusaki, A. Jorio, L. N. Coelho, R. Magalhaes-Paniago, M. A. Pimenta, Applied Physics Letters, 2006, 88, 163106.

[62] J. M. Lafferty, L. G. Rubin, Foundations of vacuum science and technology, AIP, 1999.

[63] M. H. Hablanian, High-vacuum technology: a practical guide, Vol. 111, CRC Press, 1997.

[64] A. Kutana, K. P. Giapis, The Journal of Physical Chemistry C, 2009, 113, $14721-14726$.

[65] R. E. Palmer, P. J. Rous, J. L. Wilkes, R. F. Willis, Phys. Rev. Lett., 1988, 60, 329-332. 
[66] P. H. Krupenie, Journal of physical and chemical reference data, 1972, 1, 423534.

[67] G. J. Schulz, Rev. Mod. Phys., 1973, 45, 423-486.

[68] M. J. Bojan, W. A. Steele, Langmuir, 1987, 3, 1123-1127.

[69] H. Ulbricht, G. Moos, T. Hertel, Physical Review B, 2002, 66.

[70] R. J. Chen, N. R. Franklin, J. Kong, J. Cao, T. W. Tombler, Y. Zhang, H. Dai, Applied Physics Letters, 2001, 79, 2258.

[71] J. A. Misewich, Science, 2003, 300, 783-786.

[72] M. Freitag, V. Perebeinos, J. Chen, A. Stein, J. C. Tsang, J. A. Misewich, R. Martel, P. Avouris, Nano Letters, 2004, 4, 1063-1066.

[73] M. Freitag, Y. Martin, J. A. Misewich, R. Martel, P. Avouris, Nano Letters, 2003, 3, 1067-1071.

[74] X. Qiu, M. Freitag, V. Perebeinos, P. Avouris, Nano Letters, 2005, 5, 749-752. 


\section{Appendix A Python code}

\section{A Classes}

The following defined classes were compiled into a library for the analysis procedures discussed in this manuscript. They were accompanied with the following import header:

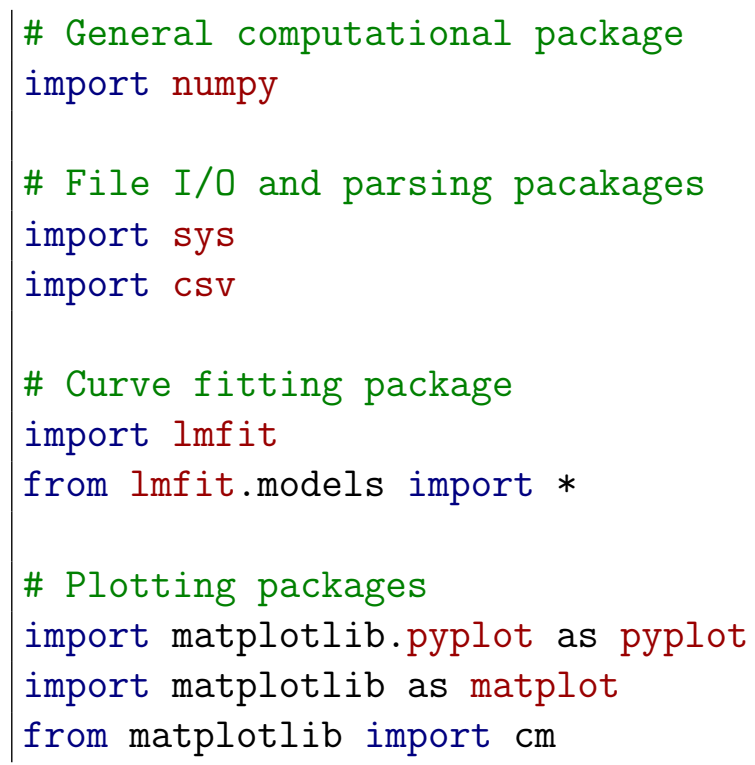

\section{A.1.1 CNTFET}

Main wrapper for containing all MEPS and Raman data as well as general metadata concerning the device. This class need only be instanciated once for an entire pool of devices.

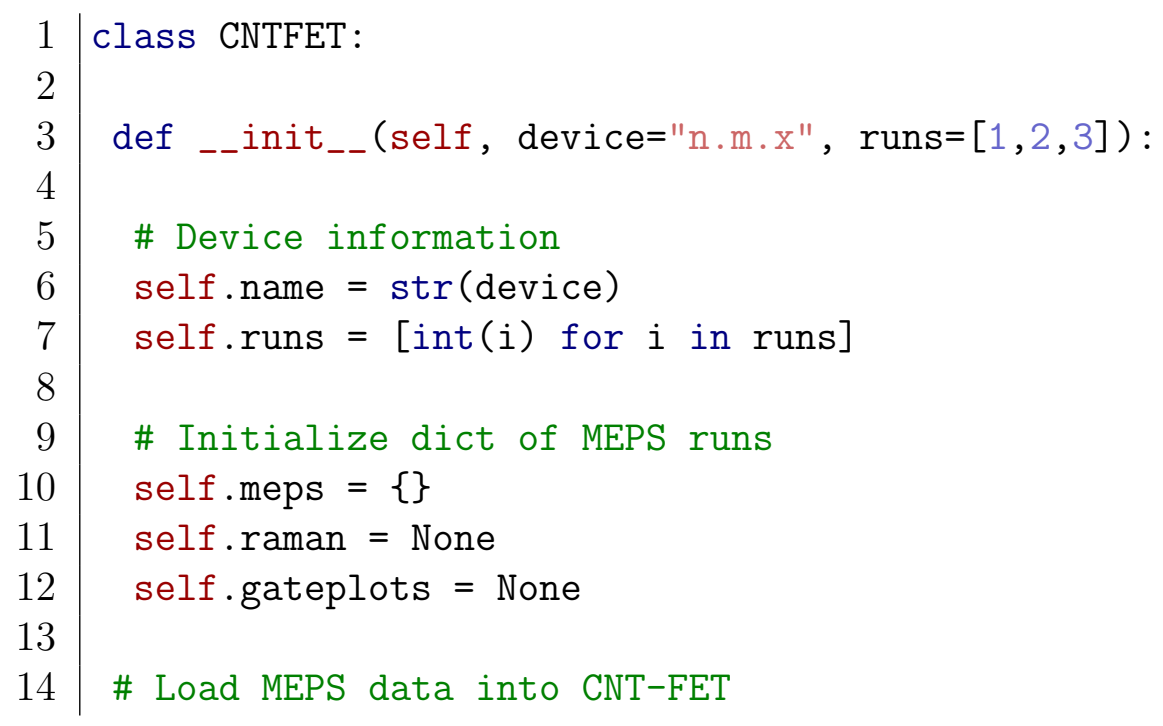




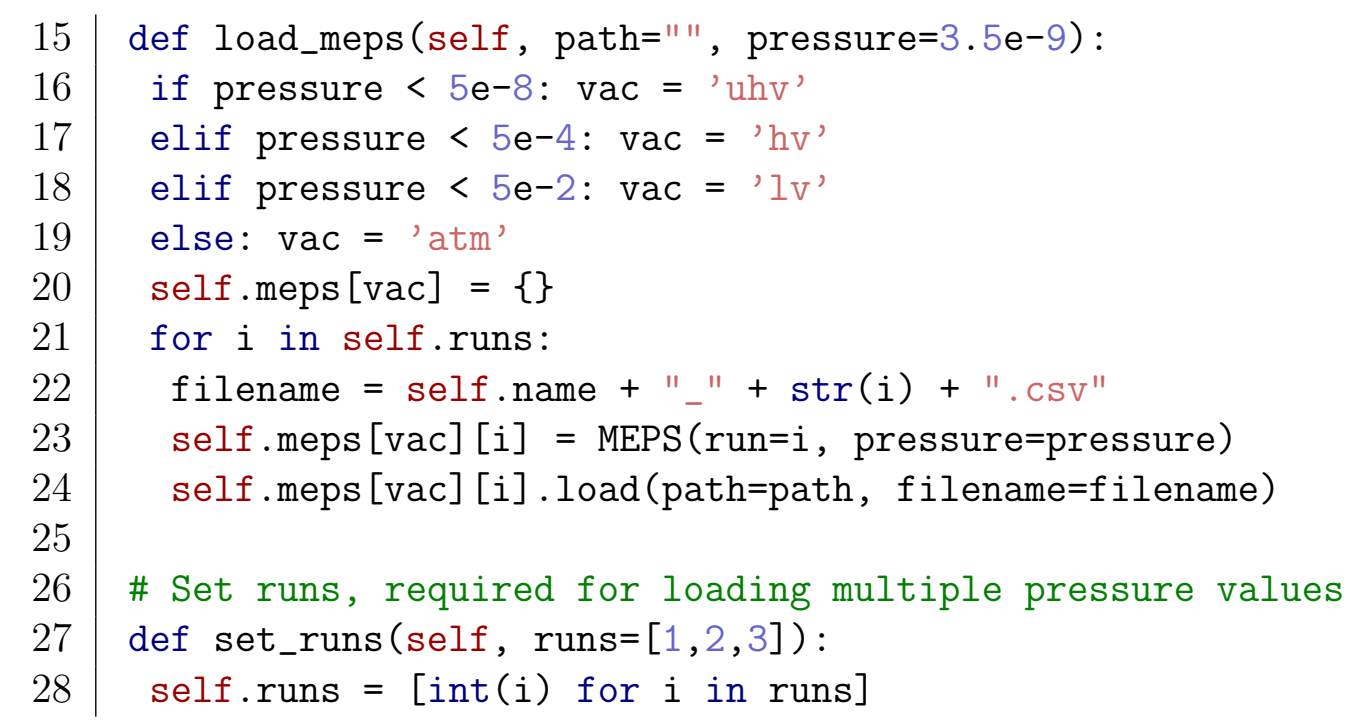

\section{A.1.2 MEPS}

Contains MEPS data. Multiple gate sweep runs can be stored in a single instance of this class but are considered trials with identical settings. This class must be instanciated for each pressure setting.

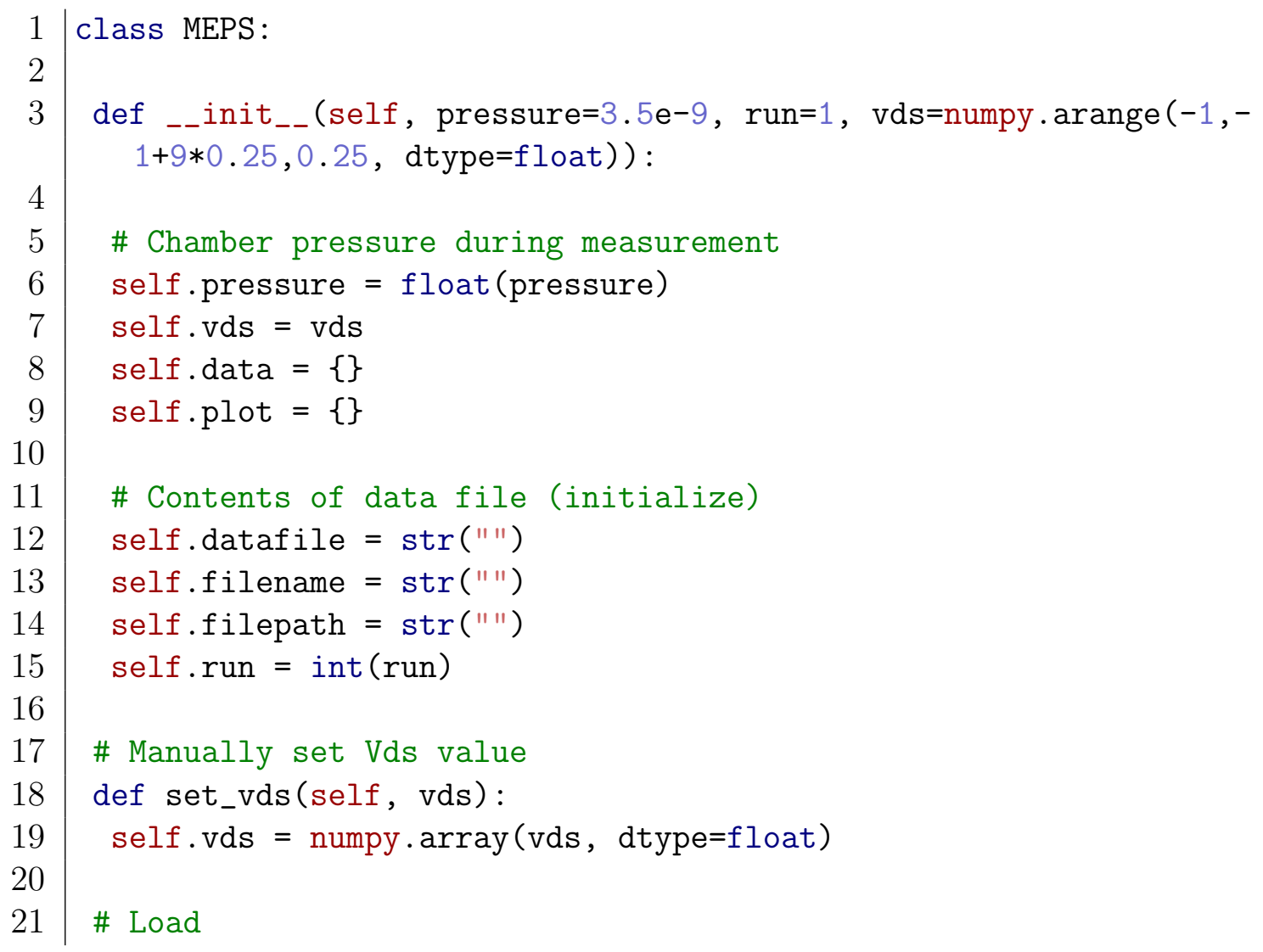




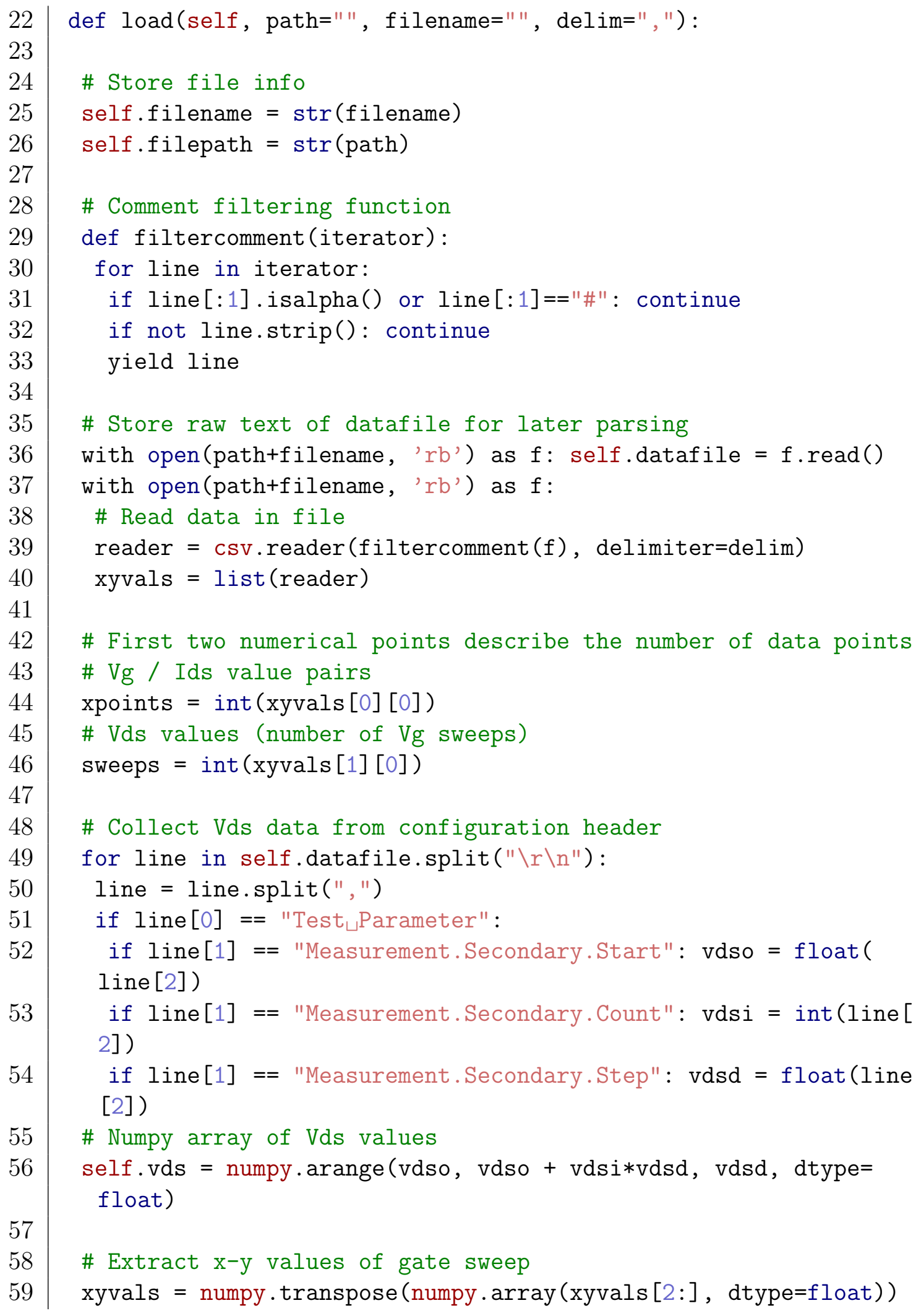




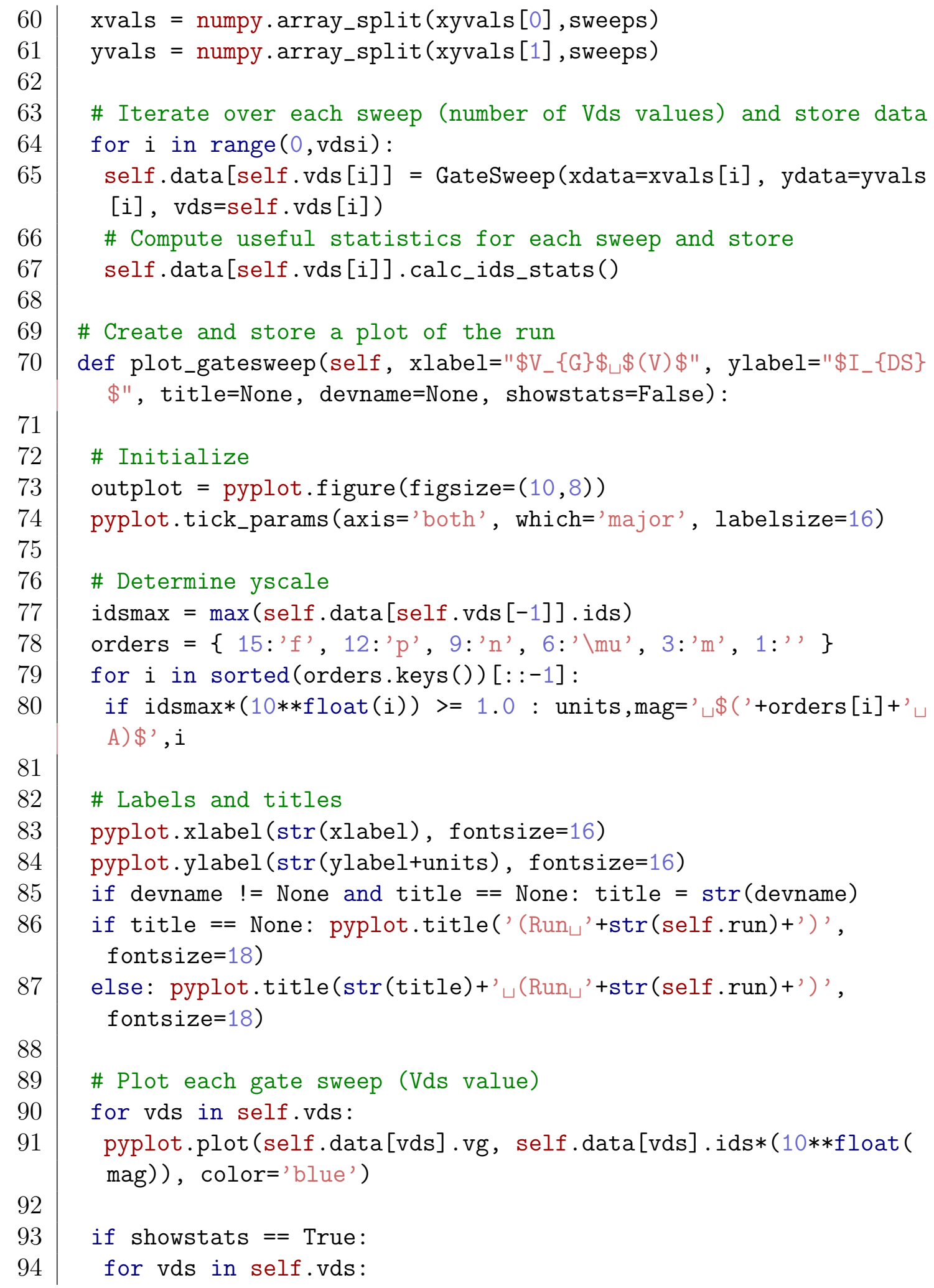




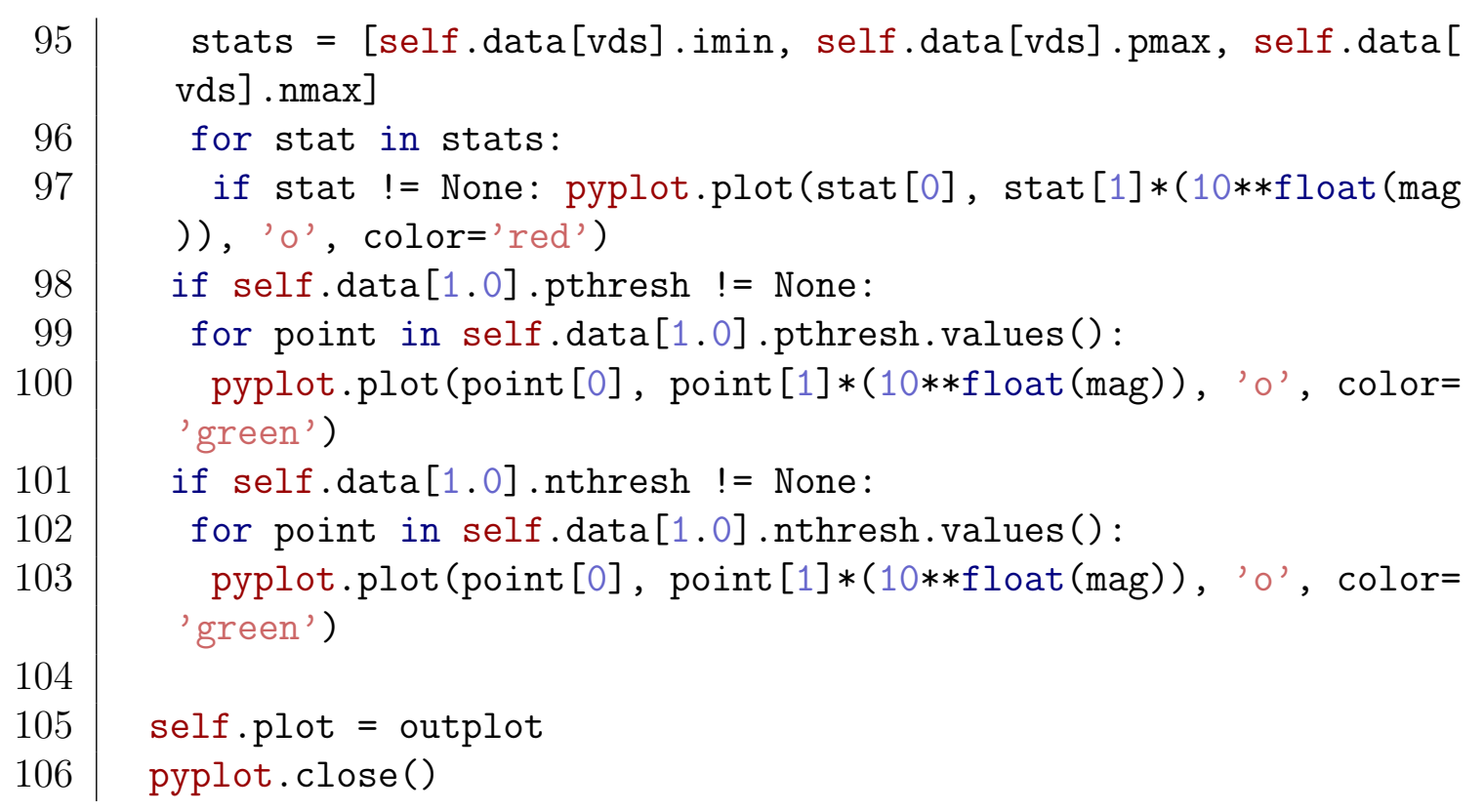

\section{A.1.3 GateSweep}

Contains a single gate sweep curve (i.e. $I_{D S}$ vs. $V_{G}$ at fixed $V_{D S}$ ). Also defines data analysis routines and stores important computed quantities. This class is automatically instanciated by the MEPS class.

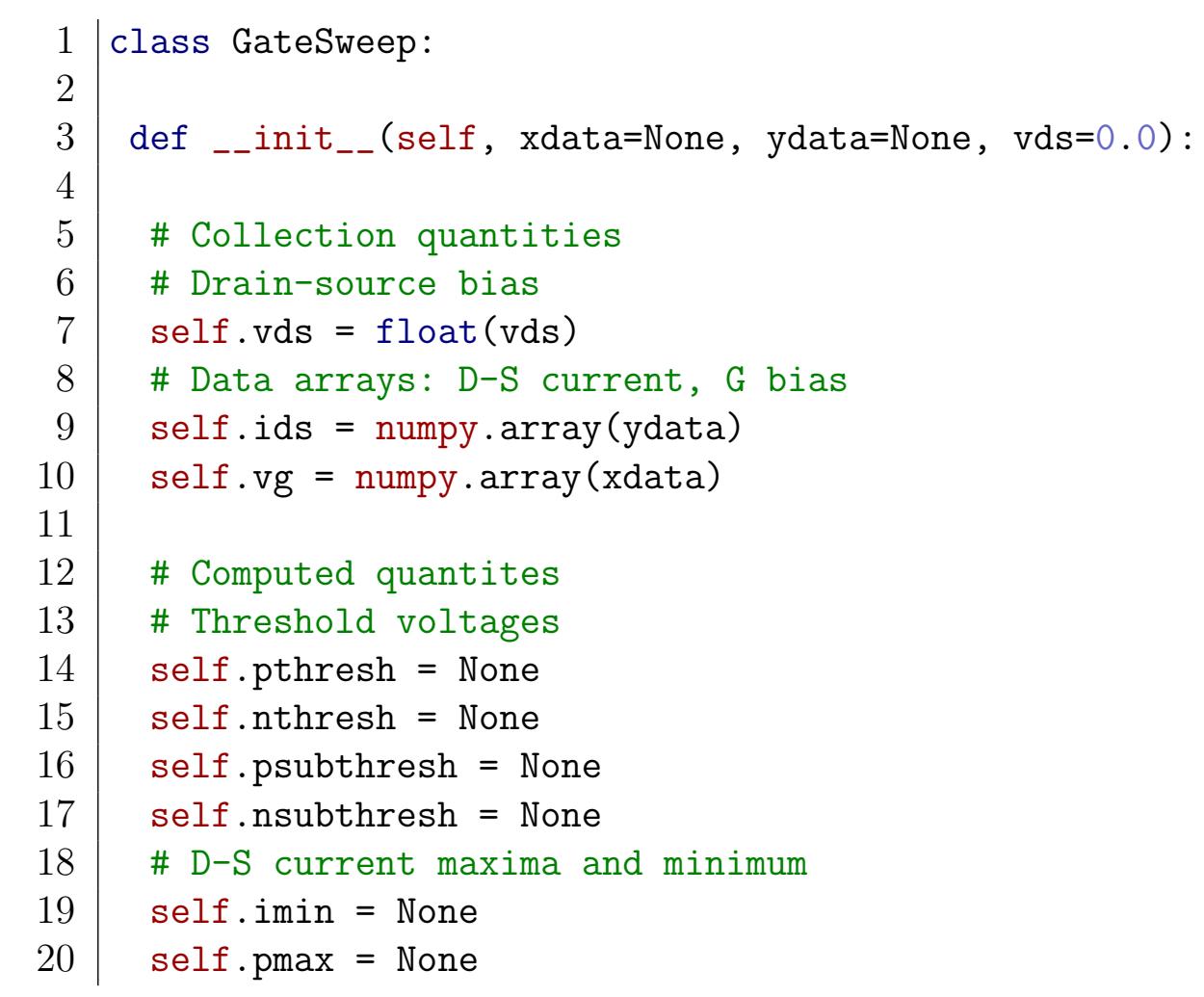




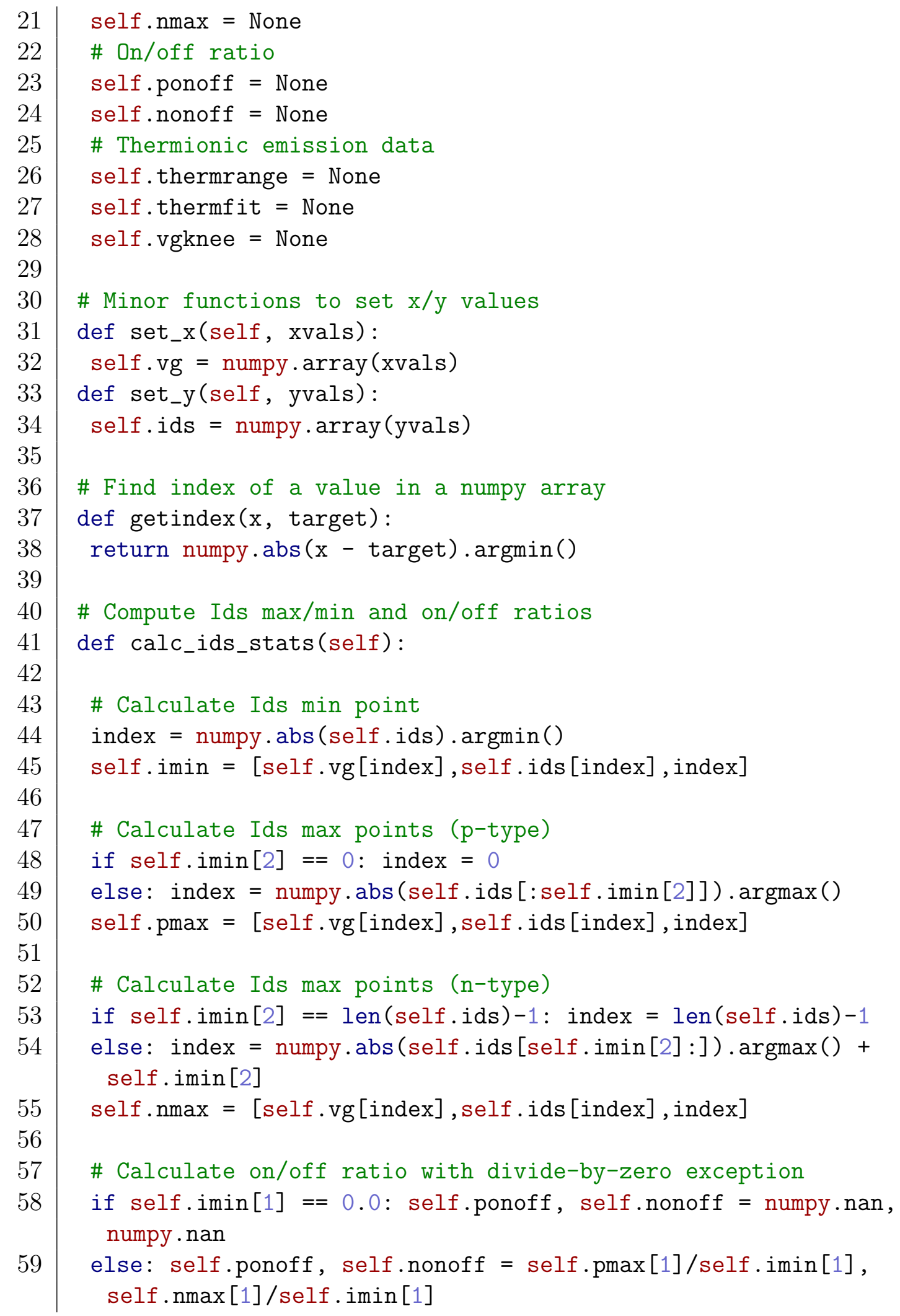




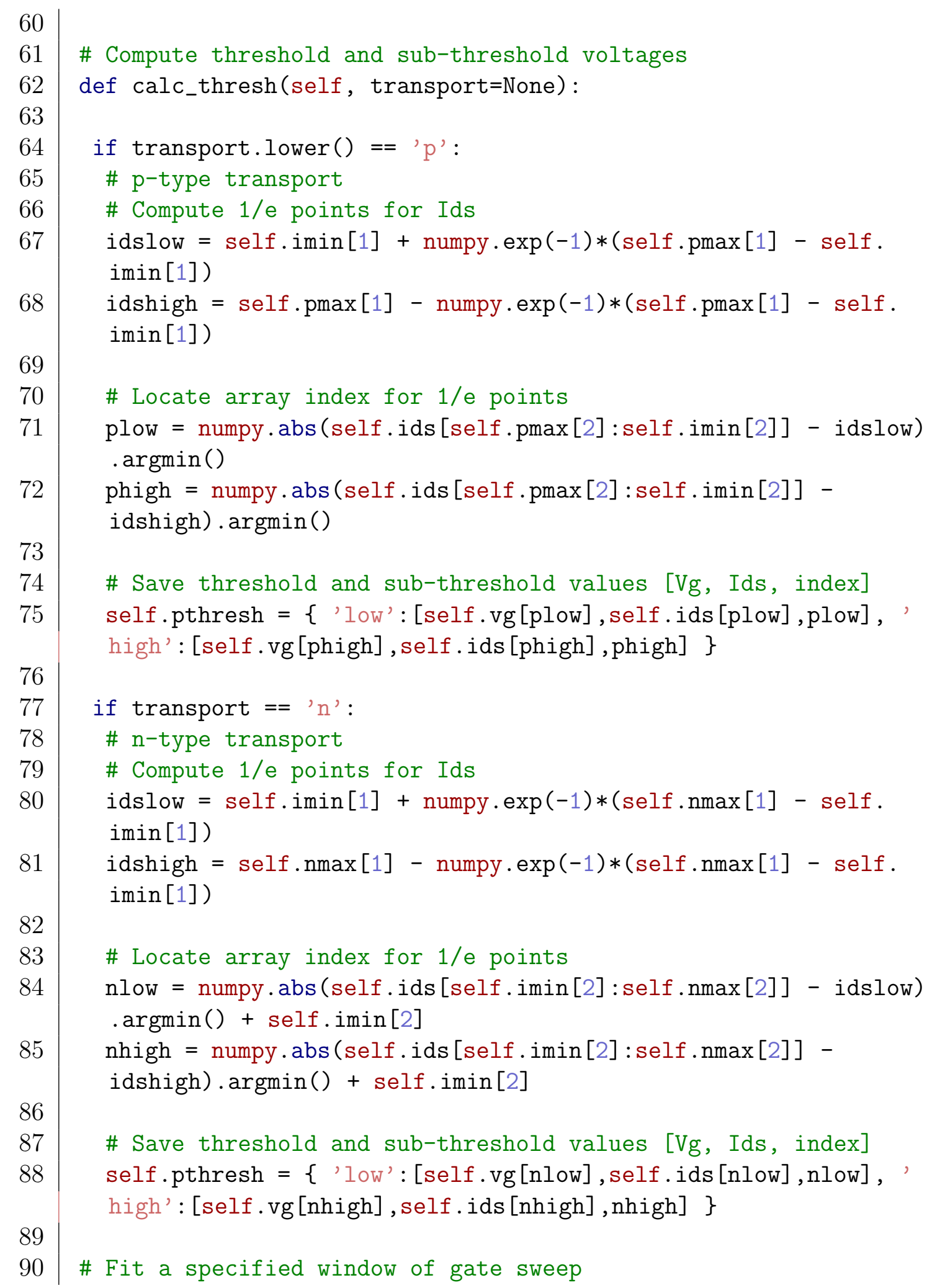




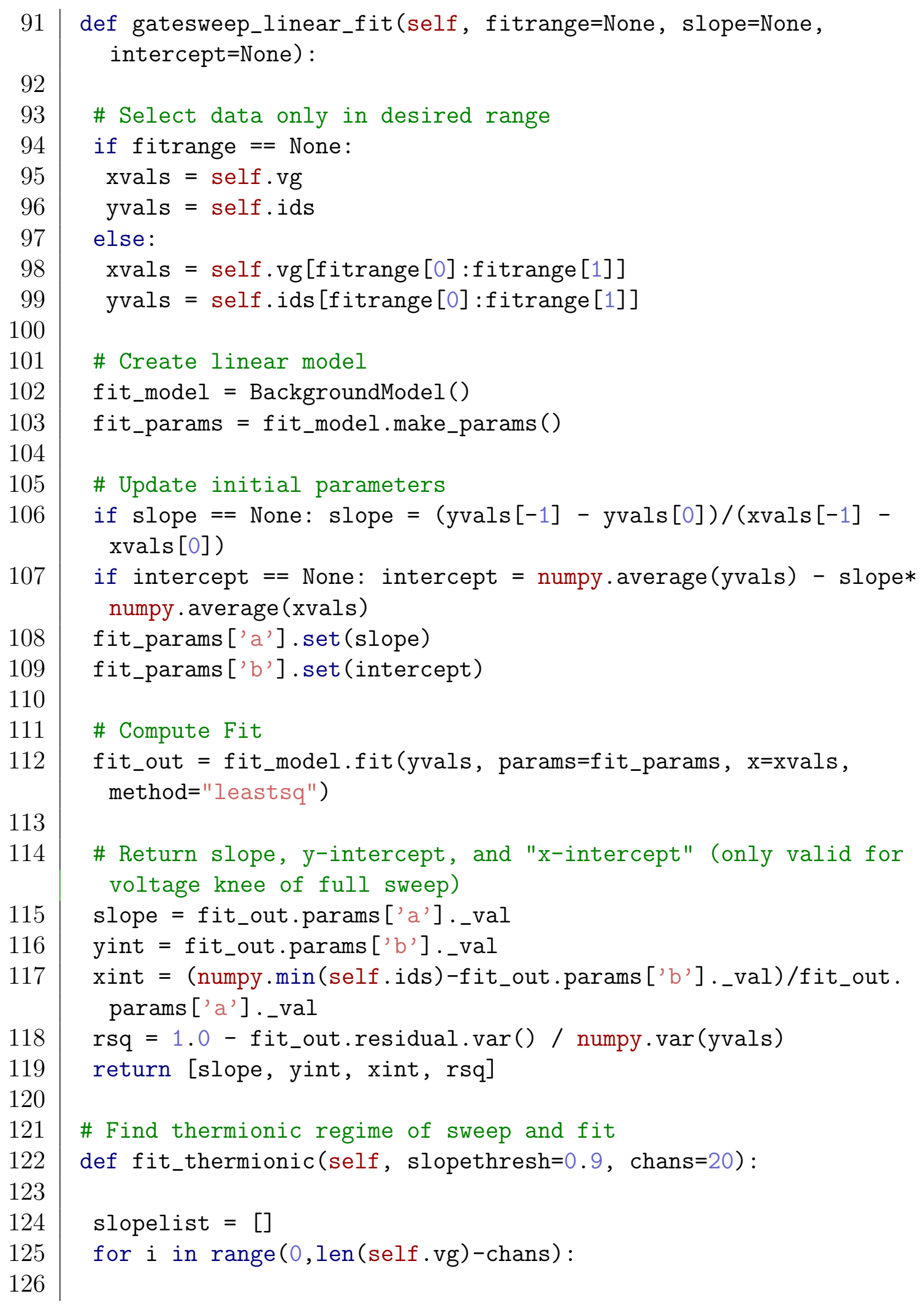




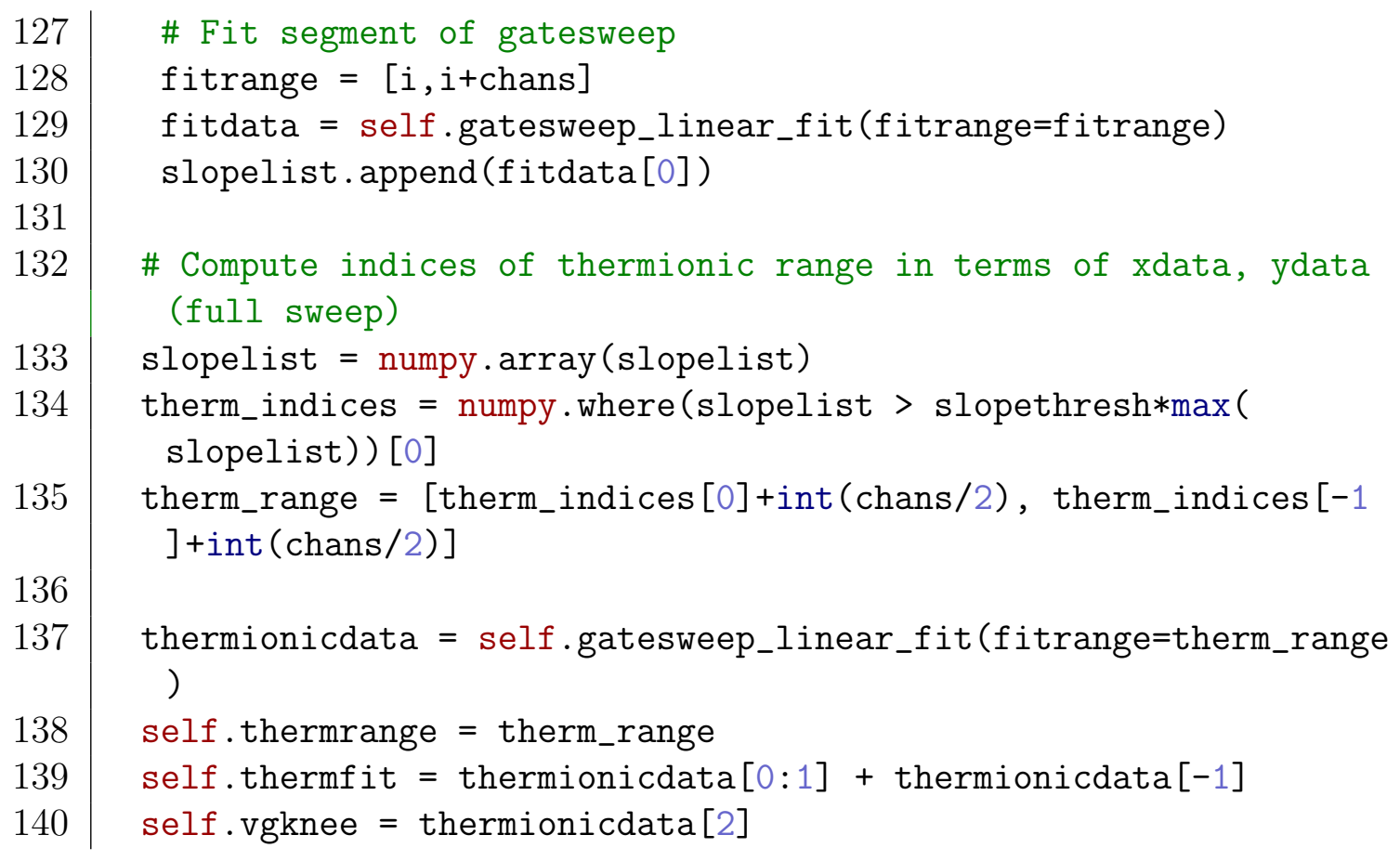

\section{A.1.4 Raman}

Contains a single Raman map for analysis. Does not perform map stitching operations. Contains all routines for region identification and statistical analysis.

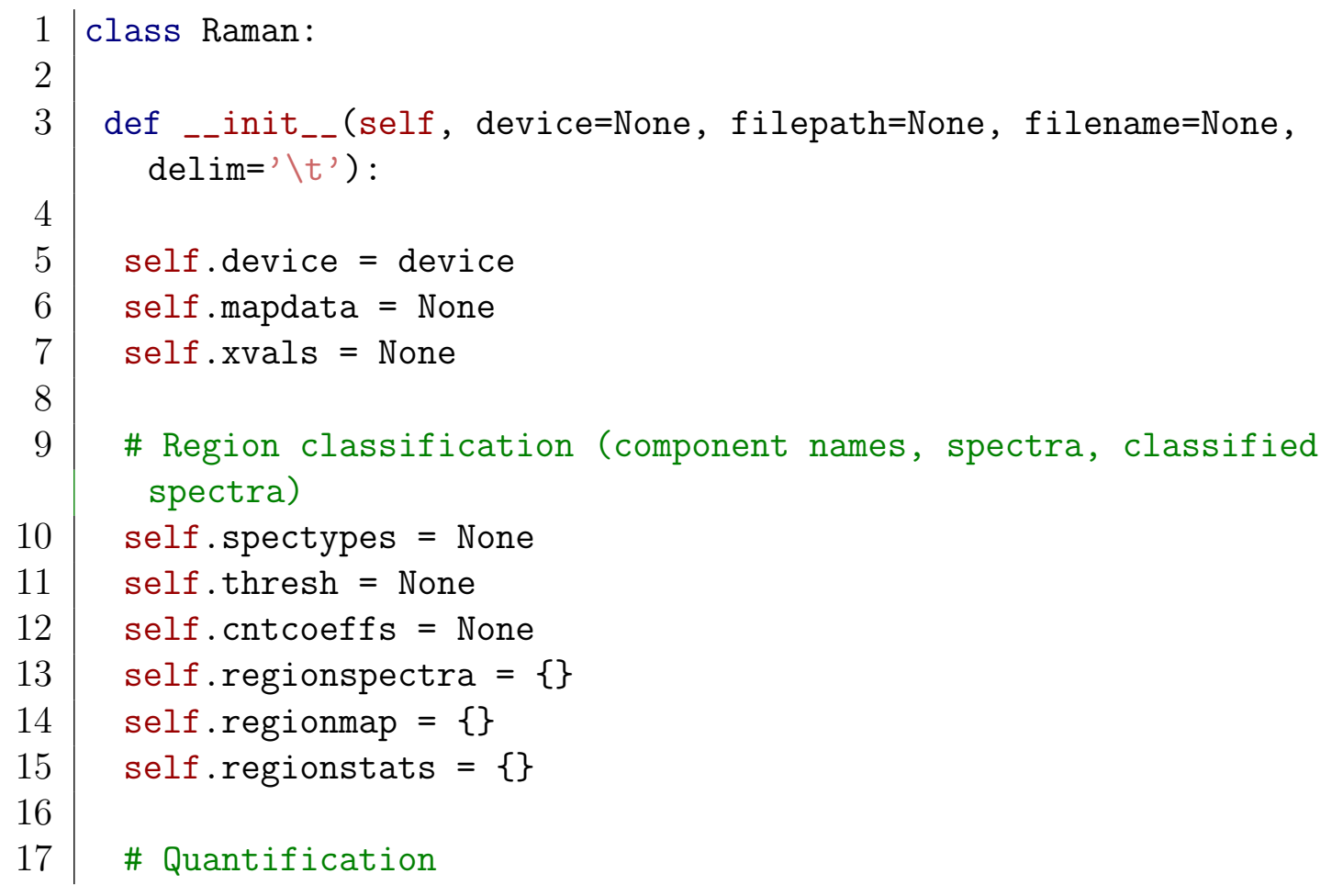




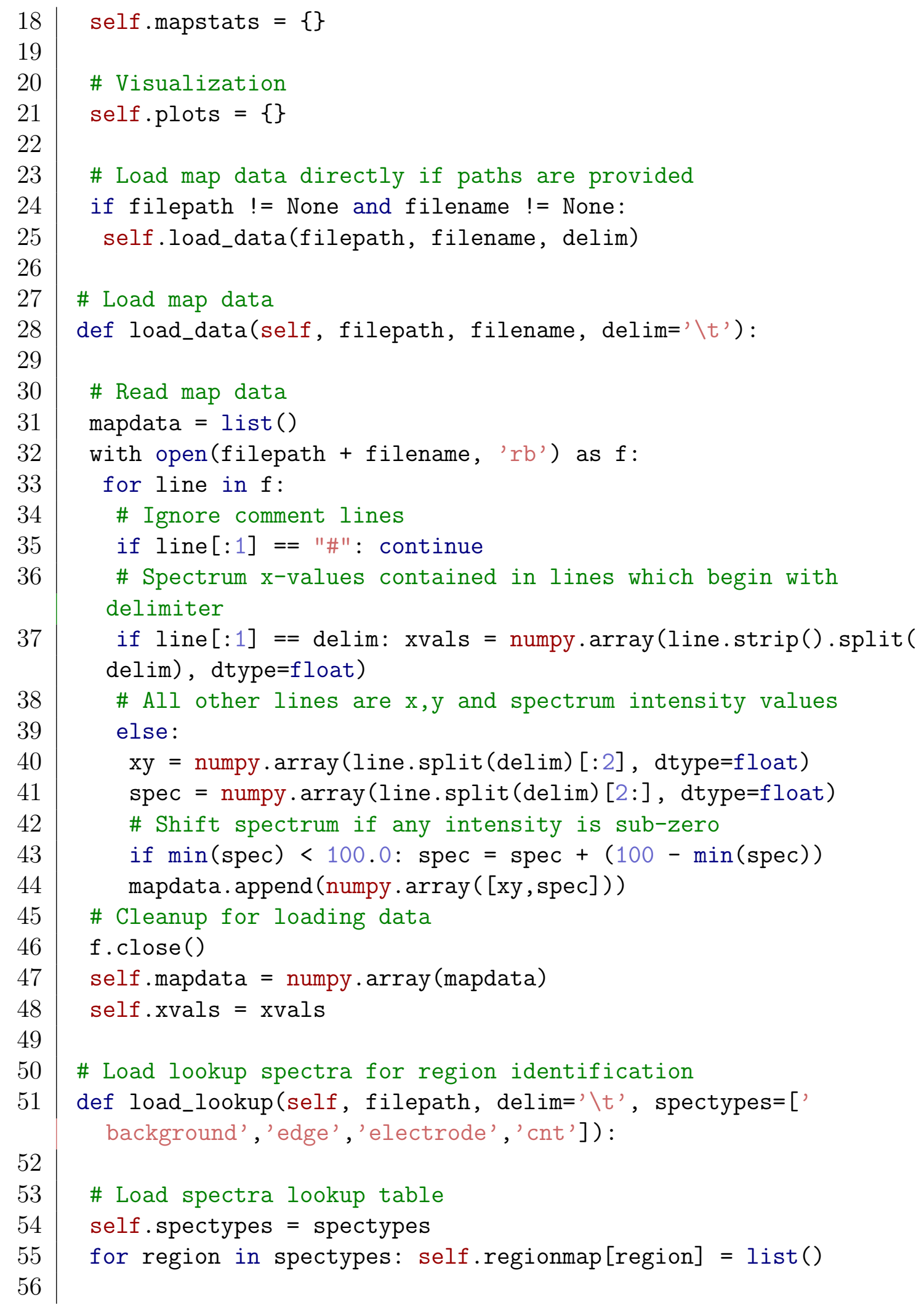




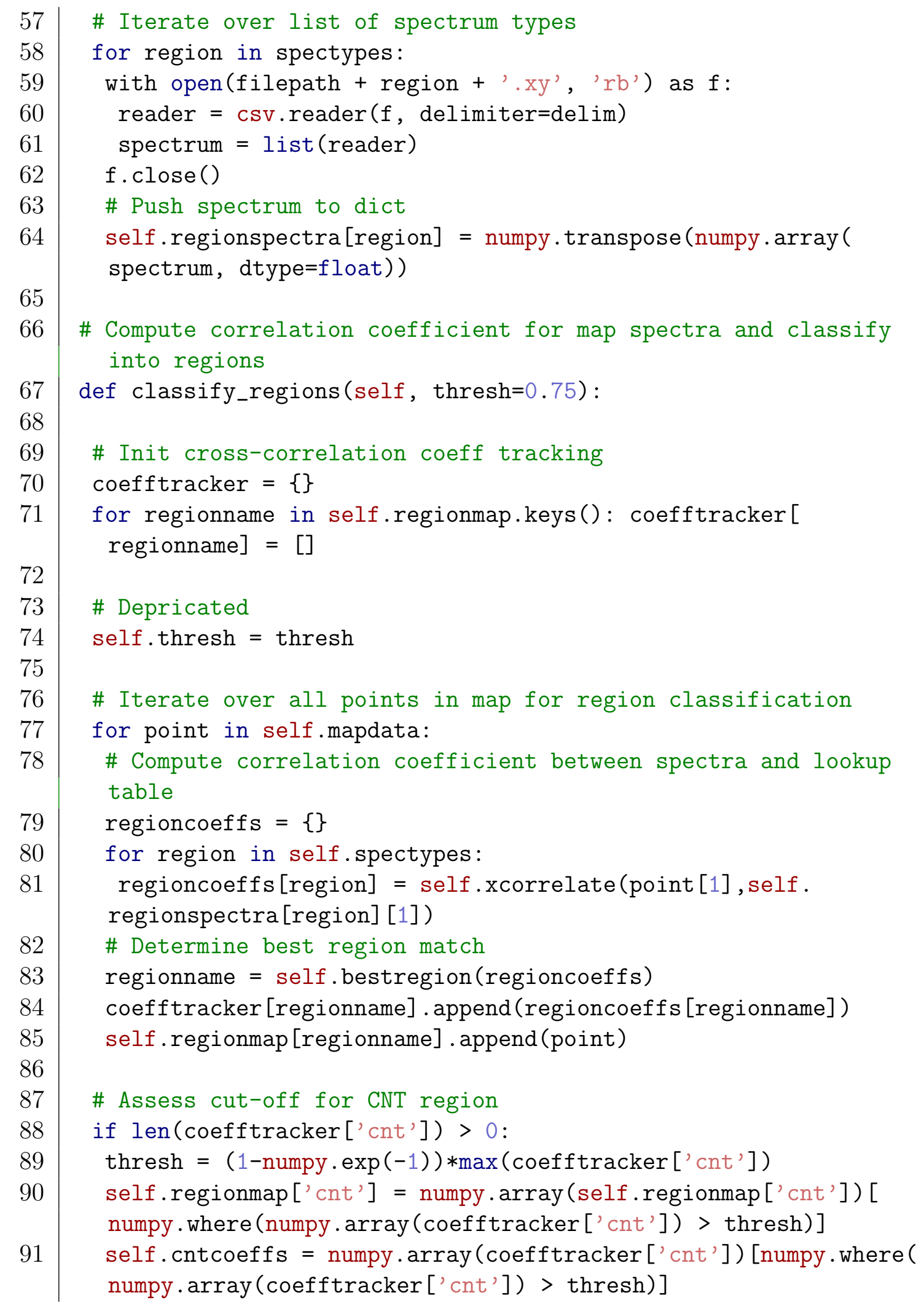




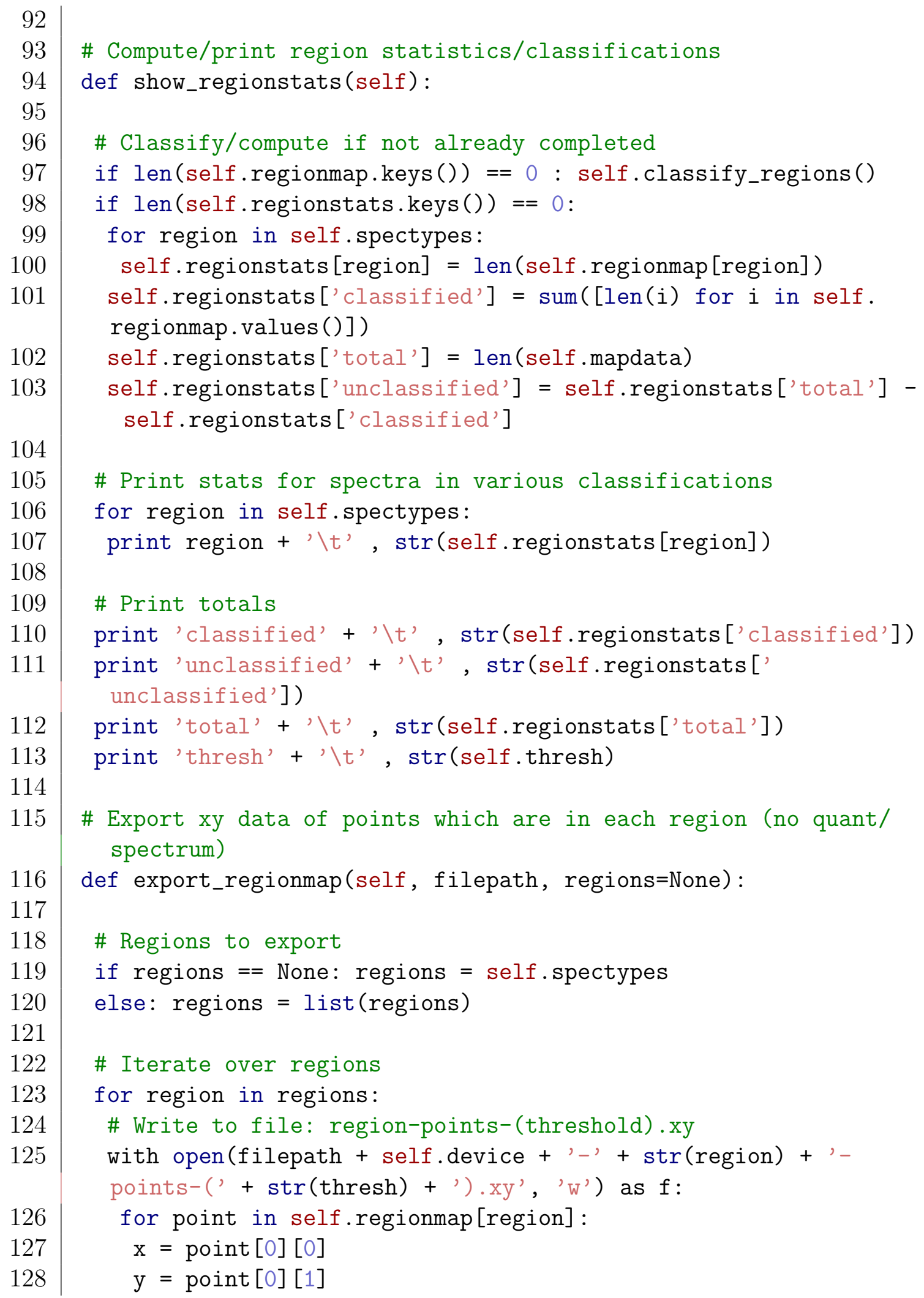


132

133

134

135

142

143

144

145

146

$$
\begin{aligned}
& \text { message }=\operatorname{str}(x)+" \backslash t "+\operatorname{str}(y)+" \backslash n " \\
& \text { f.write(message) } \\
& \text { f.close() }
\end{aligned}
$$

\# Compute spectrum stats for CNT region def calc_stats(self):

\# List of stats, initialize dumping ground for stats, peak windows

statlist $=[" \mathrm{x} "$, " $\mathrm{y}$ ", "D", "G", "2D", "D/G"]

for stat in statlist: self.mapstats[stat] = list()

drange $=[1280.0,1410.0]$

grange $=[1510.0,1670.0]$

gprange $=[2620.0,2760.0]$

\# Compute integrated intensity of D, G, and 2D peaks

for point in self.regionmap["cnt"]:

dintensity $=$ numpy.sum(self.get_peak $($ xdata=self.xvals, ydata= point [1], peakname='D'))

gintensity $=$ numpy.sum(self.get_peak $(x d a t a=s e l f . x v a l s$, ydata $=$ point [1], peakname=' $\left.G^{\prime}\right)$ )

gpintensity $=$ numpy.sum(self.get_peak(xdata=self.xvals, ydata= point [1], peakname='2D'))

self .mapstats [" $\mathrm{x} "]$. append (point [0] [0])

self .mapstats ["y"] .append (point [0] [1])

self.mapstats ["D"] . append (dintensity)

self .mapstats ["G"] . append (gintensity)

self .mapstats ["2D"] .append (gpintensity)

self .mapstats ["D/G"] . append (dintensity/gintensity)

\# Export CNT stats for each data point in CNT region

def export_cntstatmap(self, filepath):

statlist $=[" \mathrm{x}$ ", "y", "D", "G", "2D", "D/G"]

with open (filepath + self.device + '-' + 'cnt-stats.xyz', 'W') as $f$ :

f.write ('\#' + '\t'.join(statlist) + '\n')

for $i$ in range(0, len(self.mapstats[statlist[0]])):

f.write ('\t'.join(str(self.mapstats[stat][i]) for stat in statlist $\left.)+{ }^{\prime} \backslash \mathrm{n}^{\prime}\right)$ 


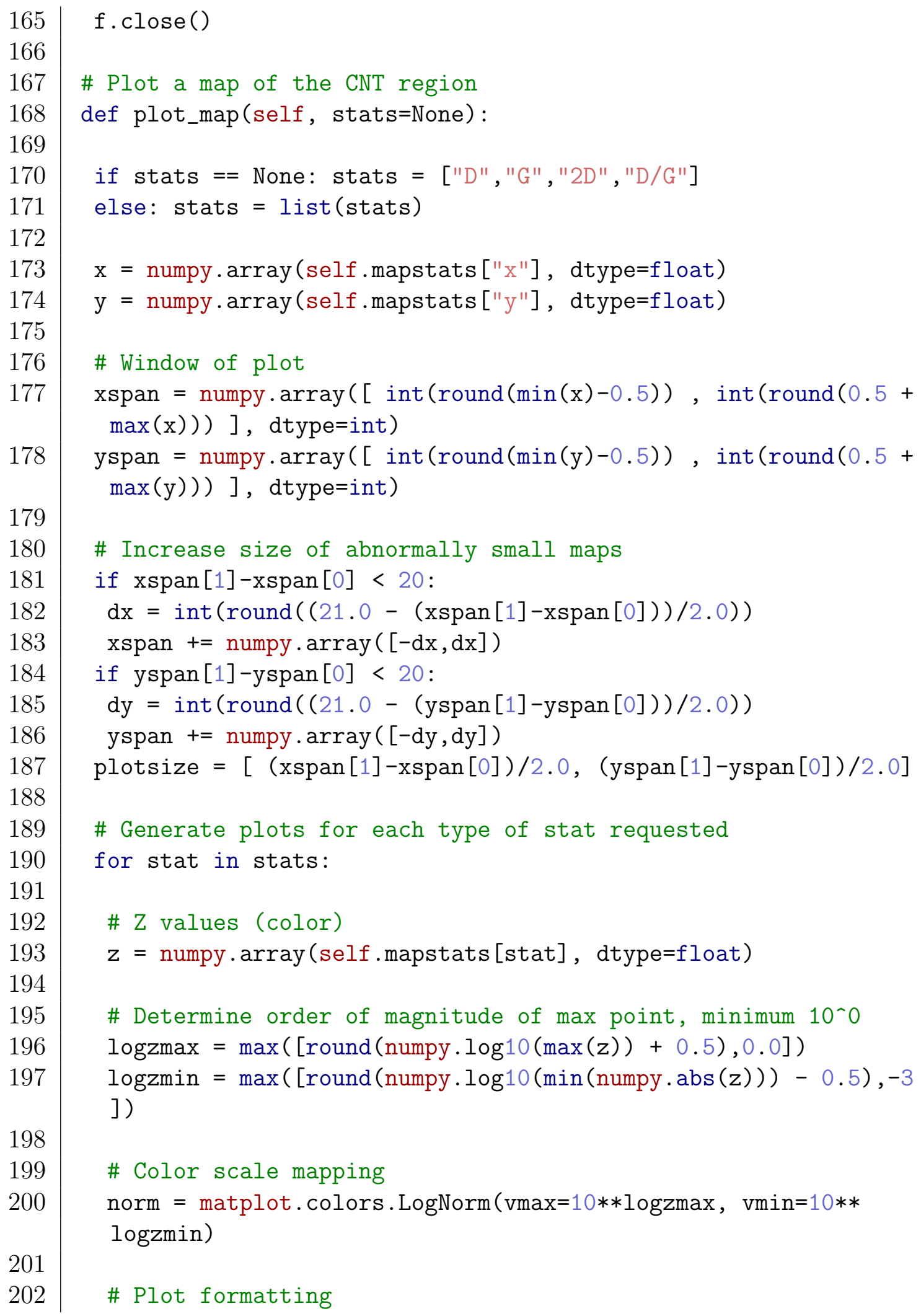




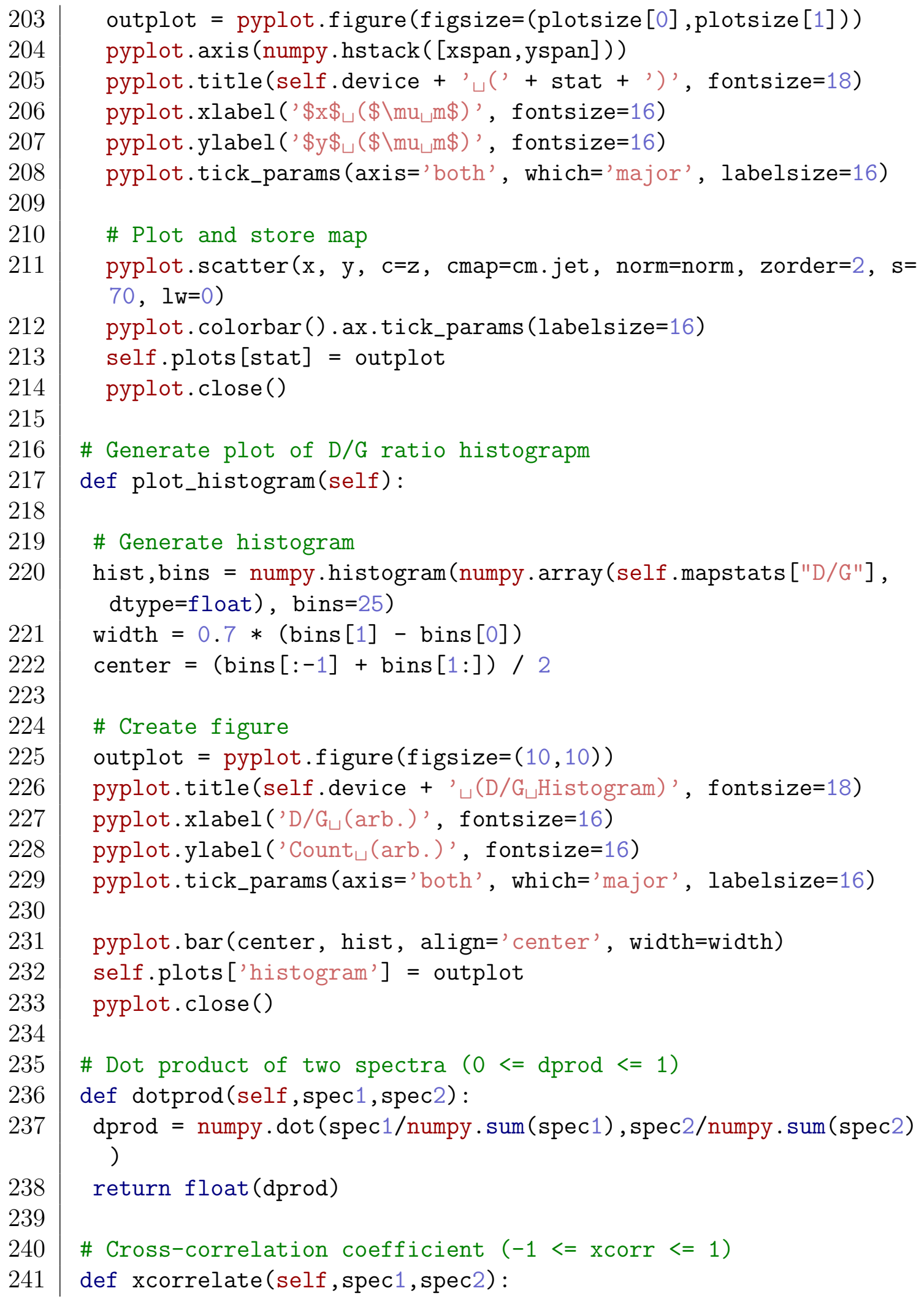




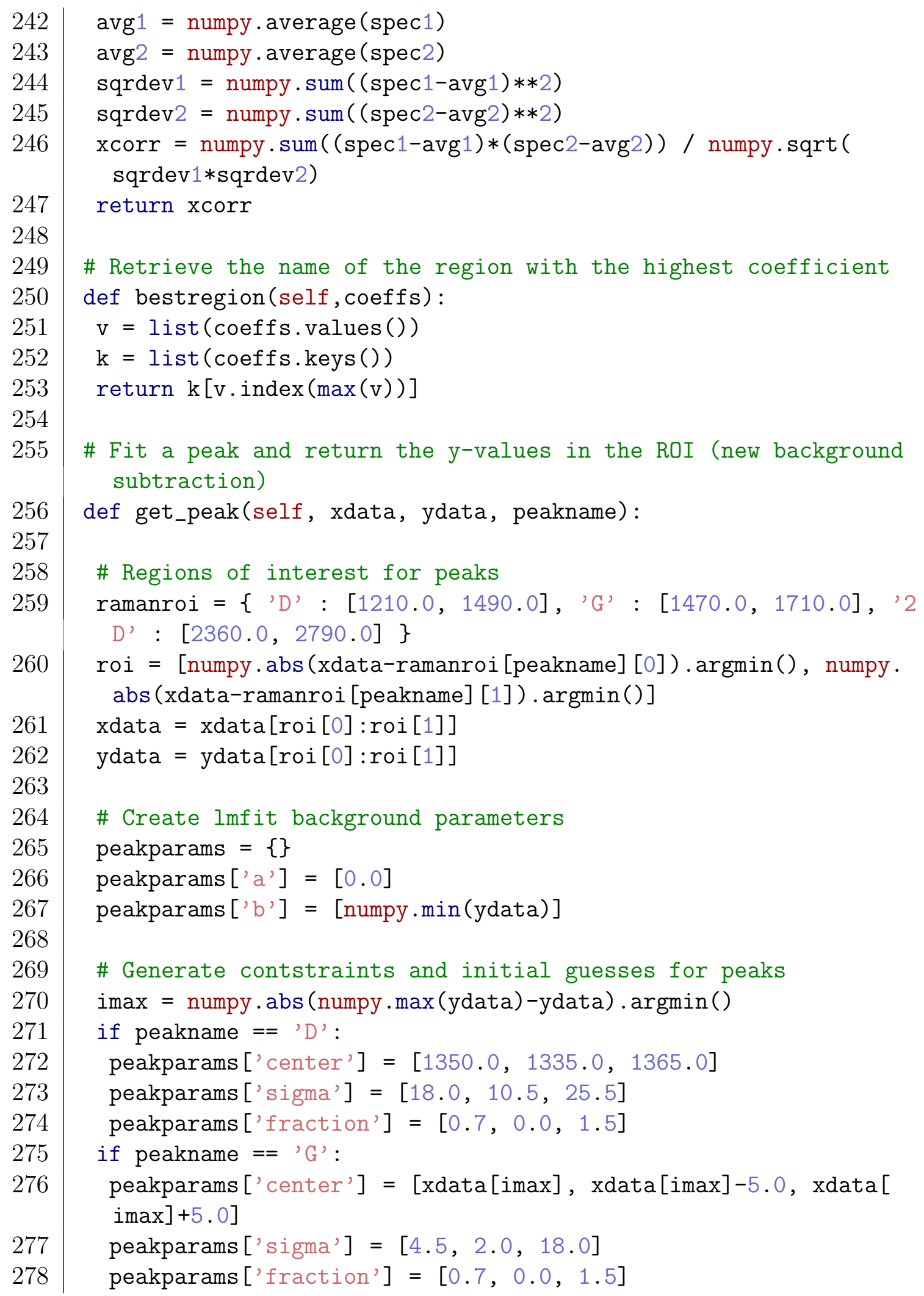




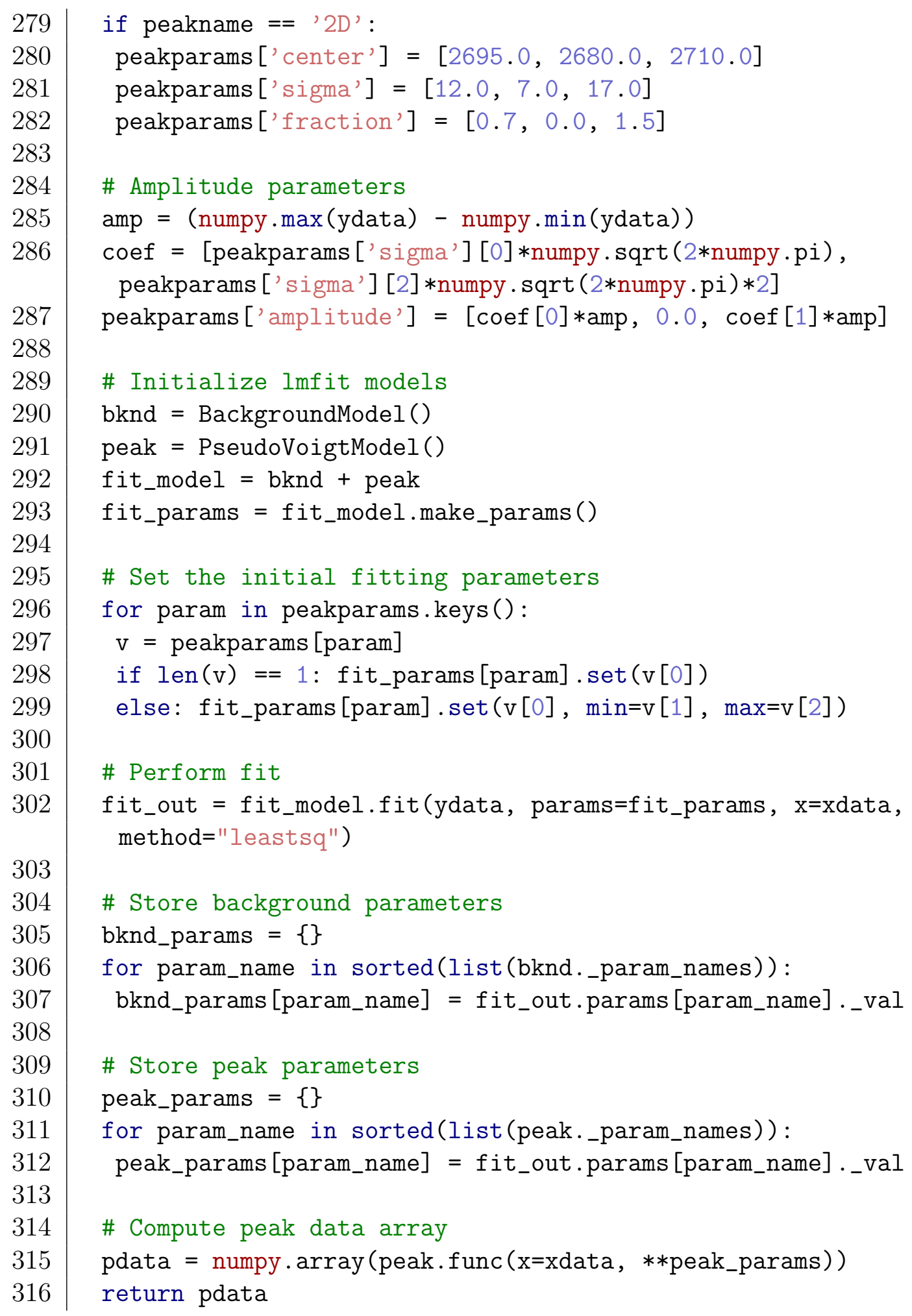




\section{A Functions}

The following defined functions were used in a number of capacities and were defined in the same library file. They are sorted into various categories.

\section{A.2.1 Curve fitting}

Custom fitting functions for use with the Lmfit Python module. Used for analyzing both MEPS and Raman data.

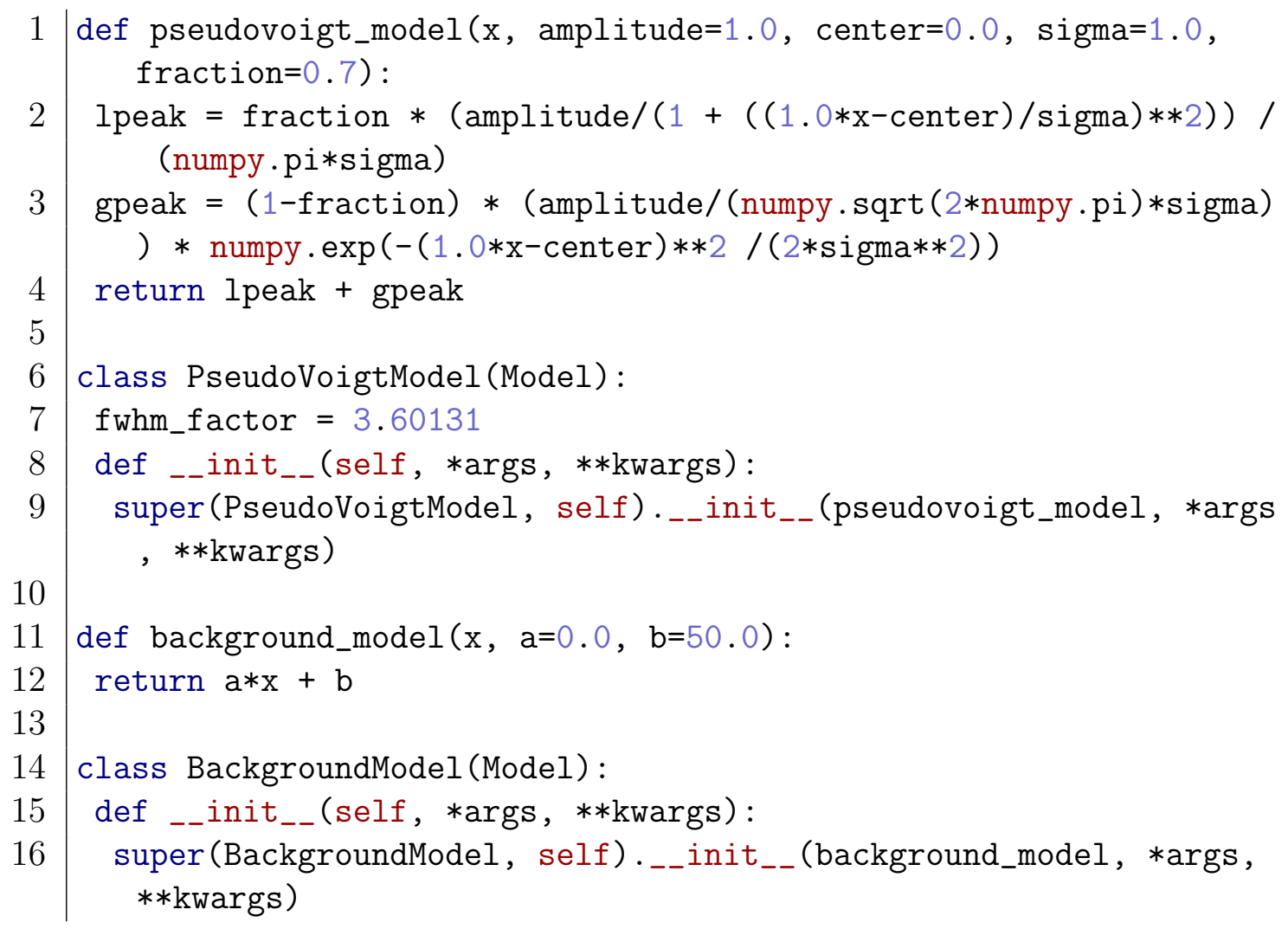

\section{A.2.2 Raman map stitching}

Functions used for stitching sequential Raman maps collected for a single device.

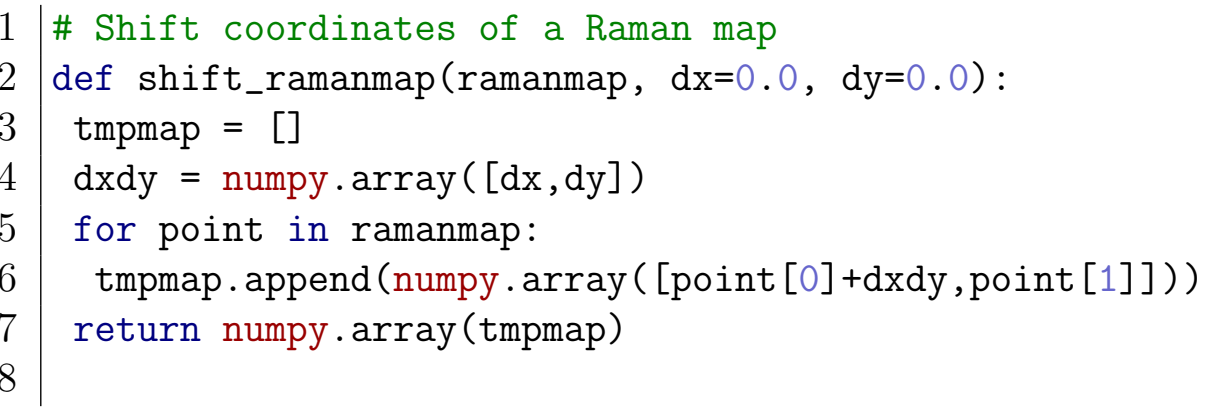




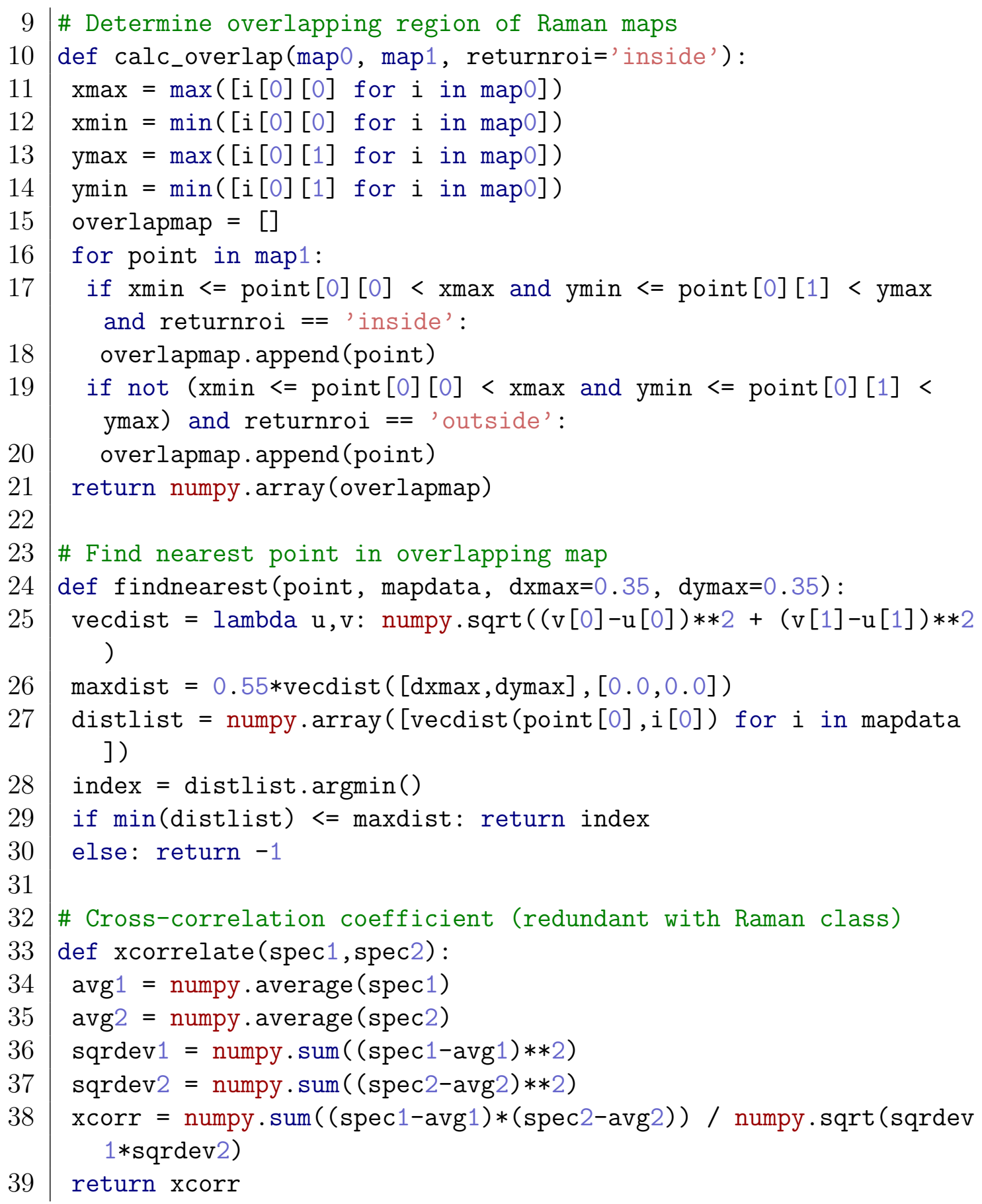

\section{A Code blocks}

The following blocks of code exist outside the previously mentioned classes and were executed as a part of the main data analysis script as a matter of convenience. 


\section{A.3.1 Raman map stitching}

This block of code performs the Raman map stitching operation. Note that an instance declared as "ramandata" was used which contains various measurement metadata (device numbers, number of map files, paths to data, etc). Some status and diagnostic data are printed to the terminal.

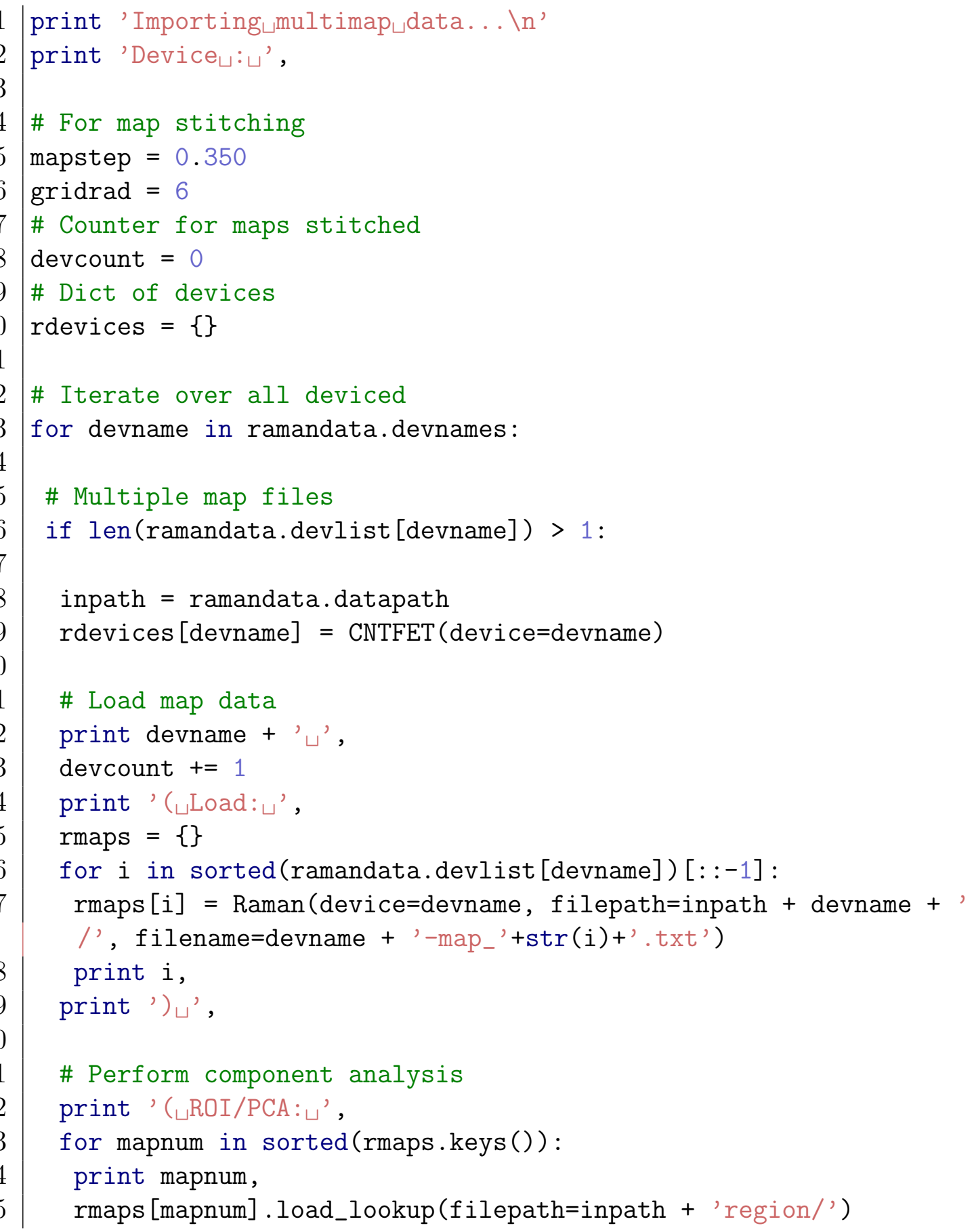




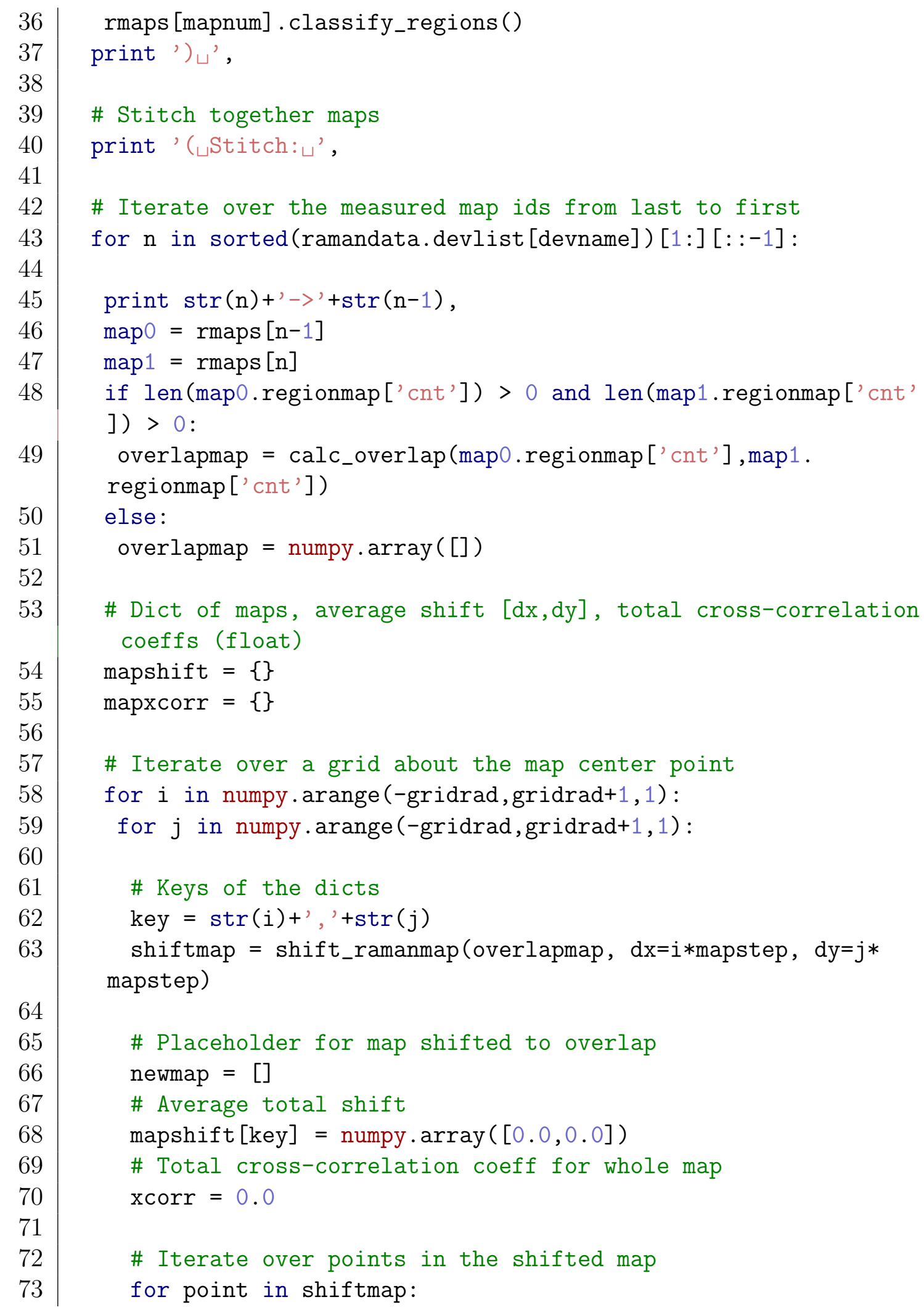




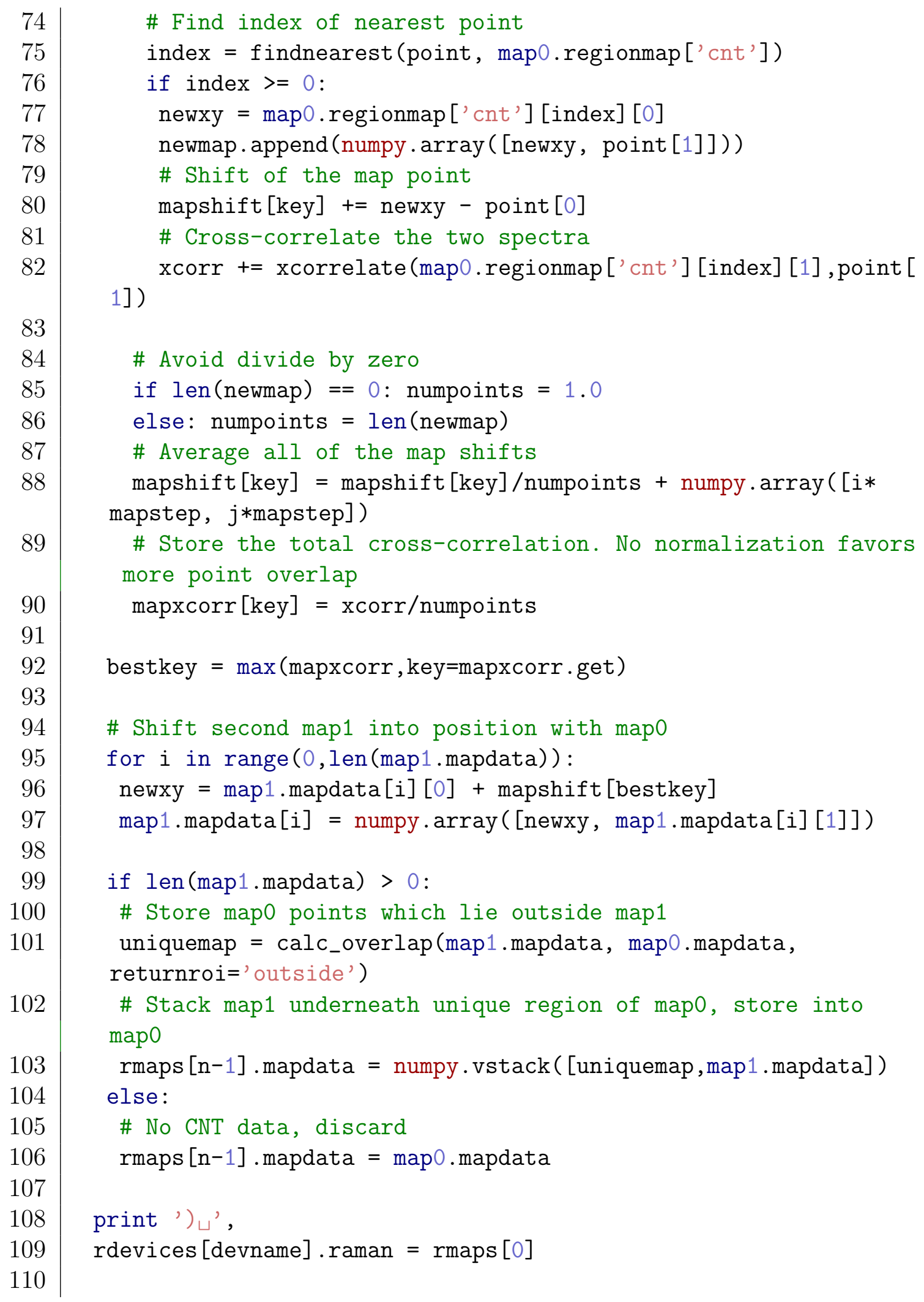


111 print ' $\backslash$ nTotal $\sqcup: \sqcup '+\operatorname{str}($ devcount)

112 print ' $\backslash \mathrm{n}$ ' 


\section{Appendix B Publications}

\section{Journal Publications}

[1] M. C. Eastman and K. S. Krane, "Neutron capture cross sections of even-mass tellurium isotopes," Physical Review C, vol. 77, no. 2, p. 024303, 2008.

[2] M. Eastman, A. Besser, Y. Chen, and J. Jiao, "Hydrothermal synthesis of Core/Shell ZnO:Mn/Mn3O4 nanowires," The Journal of Physical Chemistry C, vol. 115, no. 22, pp. 10979-10984, 2011.

[3] M. Eastman, D. Hedinger, W. Qian, W. Hudson, B. Jiang, and J. Jiao, "Raman spectroscopy and band structure of pd-hybridized multilayer graphene," Carbon, vol. 68, pp. $687-694,2014$.

\section{Conference Proceedings}

[1] M. Eastman and J. Jiao, "Electron microscopy characterization of ZnO:Mn nanowires," Microscopy and Microanalysis, vol. 15, no. Supplement, 2009.

[2] M. Eastman, H. Li, and J. Jiao, "Electron microscopy characterization of lowtemperature growth zn-doped TiO2 nanowires," MRS Online Proceedings Library, vol. 1178, 2009.

[3] M. Eastman, R. Schaller, A. Besser, and J. Jiao, "Crystalline structure and ELNES of branched Al2O3 nanowires," Microscopy and Microanalysis, vol. 16, no. S2, p. 1788-1789, 2010.

[4] M. Eastman, S. Fowler, P. Lommen, and J. Jiao, "Evaluation of monodisperse amorphous $\mathrm{SiO} 2 / \mathrm{TiO} 2$ core-shell nanoparticles," Microscopy and Microanalysis, vol. 18, no. Supplement S2, p. 1638-1639, 2012.

[5] M. Eastman, W. Qian, D. Hedinger, A. Jacobson, B. Jiang, and J. Jiao, "Band structure and spectroscopy of graphene-pd hybrids," Microscopy and Microanalysis, vol. 19, no. Supplement S2, p. 1602-1603, 2013.

[6] M. Eastman and J. Jiao, "Coloration defects in industrial-grade zirconium sponge," Microscopy and Microanalysis, vol. 21, no. S3, pp. 1779-1780, 2015. 


\section{Co-authored Publications}

[1] J. Wu, M. Eastman, T. Gutu, M. Wyse, J. Jiao, S.-M. Kim, M. Mann, Y. Zhang, and K. B. K. Teo, "Fabrication of carbon nanotube-based nanodevices using a combination technique of focused ion beam and plasma-enhanced chemical vapor deposition," Applied Physics Letters, vol. 91, no. 17, p. 173122, 2007.

[2] H. Li, M. Eastman, R. Schaller, W. Hudson, and J. Jiao, "Hydrothermal synthesis of CdS nanoparticle-decorated $\mathrm{TiO}_{2}$ nanobelts for solar cell," Journal of Nanoscience and Nanotechnology, vol. 11, pp. 8517-8521, Oct. 2011.

[3] W. Qian, Z. Chen, M. Eastman, S. Cottingham, B. A. Manhat, A. M. Goforth, and J. Jiao, "Complementary microscopy techniques applied for optimizing the structure and performance of graphene-based hybrids," Ultramicroscopy, Nov. 2011.

[4] C. Wang, Z. Jiang, L. Wei, Y. Chen, J. Jiao, M. Eastman, and H. Liu, "Photosensitization of $\mathrm{TiO} 2$ nanorods with CdS quantum dots for photovoltaic applications: A wet-chemical approach," Nano Energy, vol. 1, no. 3, pp. 440 - 447, 2012.

[5] W. Qian, R. Hao, J. Zhou, M. Eastman, B. A. Manhat, Q. Sun, A. M. Goforth, and J. Jiao, "Exfoliated graphene-supported pt and pt-based alloys as electrocatalysts for direct methanol fuel cells," Carbon, vol. 52, pp. 595-604, Feb. 2013.

[6] S.-K. Chiu, B. A. Manhat, W. J. DeBenedetti, A. L. Brown, K. Fichter, T. Vu, M. Eastman, J. Jiao, and A. M. Goforth, "Aqueous red-emitting silicon nanoparticles for cellular imaging: Consequences of protecting against surface passivation by hydroxide and water for stable red emission," Journal of Materials Research, vol. 28, no. 02, p. 216-230, 2013. 